\title{
A POINTWISE FINITE-DIMENSIONAL REDUCTION METHOD FOR A FULLY COUPLED SYSTEM OF EINSTEIN-LICHNEROWICZ TYPE.
}

\author{
BRUNO PREMOSELLI
}

\begin{abstract}
We construct non-compactness examples for the fully coupled Einstein-Lichnerowicz constraint system in the focusing case. The construction is obtained by combining pointwise a priori asymptotic analysis techniques, finite-dimensional reductions and a fixed-point argument.

More precisely, we perform a fixed-point procedure on the remainders of the expected blow-up decomposition. The argument consists of an involved finite-dimensional reduction coupled with a ping-pong method. To overcome the non-variational structure of the system, we work with remainders which belong to strong function spaces and not merely to energy spaces. Performing both the ping-pong argument and the finite-dimensional reduction therefore heavily relies on the $a$ priori pointwise asymptotic techniques of the $C^{0}$ theory, as developed in Druet-Hebey-Robert [18.
\end{abstract}

\section{INTRODUCTION}

1.1. Statement of the results. Let $(M, g)$ be a closed Riemannian manifold of dimension $n \geq 6$, where closed means here compact without boundary. We investigate non-compactness issues in strong spaces for the set of positive solutions of the Einstein-Lichnerowicz system of equations in $M$ :

$$
\left\{\begin{aligned}
\triangle_{g} u+h u & =f u^{2^{*}-1}+\frac{\left|\mathcal{L}_{g} T+\sigma\right|_{g}^{2}+\pi^{2}}{u^{2^{*}+1}} \\
\vec{\triangle}_{g} T & =u^{2^{*}} X+Y .
\end{aligned}\right.
$$

The unknowns of (1.1) are $u$, a smooth positive function in $M$, and $T$, a smooth field of 1-forms in $M$. In (1.1) we denoted by $\mathcal{L}_{g} T$ the conformal Killing derivative of $T$, whose expression in coordinates is:

$$
\mathcal{L}_{g} T_{i j}=\nabla_{i} T_{j}+\nabla_{j} T_{i}-\frac{2}{n}\left(\operatorname{div}_{g} T\right) g_{i j},
$$

and we denoted by $\vec{\triangle}_{g}$ the Lamé operator acting on 1-forms as $\vec{\triangle}_{g} T=-\operatorname{div}_{g}\left(\mathcal{L}_{g} T\right)$. In (1.2), $\nabla$ stands for the Levi-Civita connection of the metric. The second equation of (1.1) is in particular a 1-form equation. Also, in (1.1), $\triangle_{g}=-\operatorname{div}_{g}(\nabla \cdot)$ is the Laplace-Beltrami operator, $h, f, \pi$ are smooth functions in $M, \sigma$ is a smooth field of 2 -forms with $\operatorname{tr}_{g} \sigma=0$ and $\operatorname{div}_{g} \sigma=0$ and $X$ and $Y$ are smooth fields of 1 -forms in $M$. The notation $2^{*}=\frac{2 n}{n-2}$ denotes the critical exponent for the embedding of the Sobolev space $H^{1}(M)$ into Lebesgue spaces. We also assume that:

$$
f>0 \text { in } M,
$$

and that $\triangle_{g}+h$ is coercive (which is necessary in view of (1.3)).

The Einstein-Lichnerowicz system (1.1) arises in the initial-value problem in Mathematical General Relativity, as a conformal formulation of the constraint equations; see Bartnik-Isenberg [2] for the physical derivation of (1.1). In the relativistic physical case, the coefficients of (1.1) express in terms of the background physics data of the problem: for instance, in a scalar-field setting, they write as:

$$
h=c_{n}\left(S_{g}-|\nabla \psi|_{g}^{2}\right), f=c_{n}\left(2 V(\psi)-\frac{n-1}{n} \tau^{2}\right), X=-\frac{n-1}{n} \nabla \tau, Y=-\pi \nabla \psi,
$$


where $c_{n}=\frac{n-2}{4(n-1)}$. In (1.4), $V: \mathbb{R} \rightarrow \mathbb{R}$ is a potential, $\psi, \pi: M \rightarrow \mathbb{R}$ are scalar-field components, $\tau: M \rightarrow \mathbb{R}$ is the mean extrinsic curvature and $S_{g}$ is the scalar curvature of $g$. In view of (1.4), the focusing assumption (1.3) naturally arises as one considers non-trivial non-gravitational physics data.

In the focusing case (1.3), existence results for (1.1) were first obtained in Hebey-Pacard-Pollack [26], under the assumption $X \equiv 0$ that decouples (1.1); multiplicity results were then obtained in Ma-Wei [35], Premoselli [38] and, for specific physical cases, in Holst-Meier [30], Chruściel-Gicquaud 9] and Bizoń-Pletka-Simon [6]. For the fully coupled system - when $X \not \equiv 0$ - existence results are in Premoselli [37] and Gicquaud-Nguyen [24.

A more satisfactory picture of system (1.1) is obtained through the analysis of its stability issues. Following the general definition given in Druet 15 (see also Hebey 25), we say that system (1.1) is stable if, for any sequence $\left(h_{k}, f_{k}, \pi_{k}, \sigma_{k}, X_{k}, Y_{k}\right)_{k}$ of coefficients converging towards $(h, f, \pi, \sigma, X, Y)$ as $k \rightarrow+\infty$ in some strong topology (to be precised), and for any sequence $\left(u_{k}, T_{k}\right)_{k}$ of solutions of:

$$
\left\{\begin{aligned}
\triangle_{g} u_{k}+h_{k} u_{k} & =f_{k} u_{k}^{2^{*}-1}+\frac{\left|\mathcal{L}_{g} T_{k}+\sigma_{k}\right|_{g}^{2}+\pi_{k}^{2}}{u_{k}^{2^{*}+1}} \\
\vec{\triangle}_{g} T_{k} & =u_{k}^{2^{*}} X_{k}+Y_{k},
\end{aligned}\right.
$$

with $u_{k}>0$, there holds, up to a subsequence and up to elements in the kernel of $\mathcal{L}_{g}$, that $\left(u_{k}, T_{k}\right)_{k}$ converges to some positive solution $\left(u_{0}, T_{0}\right)$ of (1.1) in $C^{1, \eta}(M)$ for all $0<\eta<1$. One defines the compactness of (1.1) analogously, by taking a constant sequence of coefficients $\left(h_{k}, f_{k}, \pi_{k}, \sigma_{k}, X_{k}, Y_{k}\right)_{k}=$ $(h, f, \pi, \sigma, X, Y)$. In the focusing case (1.3) and for the decoupled system (when $X \equiv 0$ ), stability results were first obtained in Druet-Hebey [16, and then in Hebey-Veronelli [28, and Premoselli [38. For the fully coupled case $X \not \equiv 0$, the stability of (1.1) has been investigated in Druet-Premoselli 21] and Premoselli [39] on locally conformally flat manifolds. It is shown in 39] that for any $n \geq 6$, equation (1.1) is stable in the $C^{2}$ topology as soon as $\pi \not \equiv 0$ and:

$$
\text { either } \quad X \text { and } \nabla f \text { have no common zero in } M \quad \text { or } \quad h-c_{n} S_{g}+\frac{(n-2)(n-4)}{8(n-1)} \frac{\triangle_{g} f}{f}<0,
$$

where $S_{g}$ denotes the scalar curvature of $g$. Stability actually holds for a slightly weaker topology, see 21] and 39. The stability of the decoupled version of (1.1) was used in 38, to describe multiplicity issues in small dimensions. In the physical case, where the coefficients are given by (1.4), the stability and the instability of (1.1) relate to the relevance of the conformal method from which it arises. We refer to the discussion in 39. In the opposite direction, the only known instability result for (1.1) has been obtained by Premoselli-Wei 40 in the decoupled case $X \equiv 0$, and for the physical choice of coefficients given by (1.4). The motivation in 40] was to obtain explicit physical counter-examples to the stability of the conformal method.

In this work, we address the instability behavior of system (1.1) from a different perspective: we leave the physical case treated in [40] aside but consider instead the fully coupled case $X \not \equiv 0$ of (1.1). As a direct counterpart of the results in [21] and [39, we obtain non-compactness results for system (1.1) in any dimension $n \geq 6$ as soon as (1.6) is not satisfied. Our main result states as follows:

Theorem 1.1. Let $(M, g)$ be a closed Riemannian manifold of dimension $n \geq 6$ of positive Yamabe type and possessing no non-trivial conformal Killing fields. There exist regular coefficients $(h, f, \pi, \sigma, X, Y)$, with $\triangle_{g}+h$ coercive, $f>0, \pi \not \equiv 0$ and $X \not \equiv 0$ such that the associated system of equations (1.1):

$$
\left\{\begin{aligned}
\triangle_{g} u+h u & =f u^{2^{*}-1}+\frac{\left|\mathcal{L}_{g} T+\sigma\right|_{g}^{2}+\pi^{2}}{u^{2^{*}+1}} \\
\vec{\triangle}_{g} T & =u^{2^{*}} X+Y
\end{aligned}\right.
$$


possesses a blowing-up sequence of solutions $\left(u_{k}, T_{k}\right)_{k}$, that is satisfying $\left\|u_{k}\right\|_{L^{\infty}(M)} \rightarrow+\infty$ and $\left\|\mathcal{L}_{g} T_{k}\right\|_{L^{\infty}(M)} \rightarrow+\infty$ as $k \rightarrow+\infty$. Also, the $u_{k}$ are positive, possess a single blow-up point and blow-up with a non-zero limit profile.

Theorem 1.1 is a non-compactness result, and therefore also an instability result. The explicit expression of the coefficients $(h, f, \pi, \sigma, X, Y)$, their regularity as well as the generality of the construction are discussed in Section 2 below. For $n \geq 7$, these coefficients do not satisfy (1.6), thus establishing its sharpness in the coupled case. A conformal Killing field is a field of 1-forms $X$ in $M$ satisfying $\mathcal{L}_{g} X=0$. The assumption that $(M, g)$ possesses no non-trivial conformal Killing fields is generic as shown in Beig-Chruściel-Schoen [3] and implies, since $M$ is closed, that $\vec{\triangle}_{g}$ has no kernel.

A striking consequence of Theorem 1.1] is the existence of an infinite number of solutions of (1.1):

Corollary 1.2. Let $(M, g)$ be a closed Riemannian manifold of dimension $n \geq 6$ of positive Yamabe type and possessing no non-trivial conformal Killing fields. There exist regular coefficients $(h, f, \pi, \sigma, X, Y)$, with $\triangle_{g}+h$ coercive, $f>0, \pi \not \equiv 0$ and $X \not \equiv 0$ such that the system (1.1) possesses an infinite number of solutions.

In the fully coupled case case $X \not \equiv 0$ that we investigate here, system (1.1) is of a different nature than in the physical decoupled case of [40. In particular, (1.1) possesses no variational structure, exhibits supercritical nonlinearities and is not well-posed for $(u, W)$ in the energy space $H^{1}(M)$. To prove Theorem 1.1 we thus develop a pointwise finite-dimensional reduction method, which combines a priori pointwise asymptotic analysis techniques with a variational Lyapunov-Schmidt-type approach in order to perform an involved ping-pong method in strong spaces. We explain in detail the method and the strategy of proof in the next sub-section.

1.2. Strategy of the proof of Theorem 1.1, In the fully coupled case $X \not \equiv 0$, system (1.1) exhibits a strong nonlinear coupling via the $\left(\left|\mathcal{L}_{g} T+\sigma\right|_{g}^{2}+\pi^{2}\right) u^{-2^{*}-1}$ term, as well as a super-critical nonlinearity in the right-hand side of the 1-form equation. Hence (1.1) does not possess a well-posed variational formulation in $H^{1}(M)$. If one only assumes that $u \in H^{1}(M)$, the right-hand side of the 1form equation is merely bounded in $L^{1}(M)$ which yields no integral control on $\left(\left|\mathcal{L}_{g} T+\sigma\right|_{g}^{2}+\pi^{2}\right) u^{-2^{*}-1}$ whatsoever. This is a serious obstacle to combining a ping-pong approach with a finite-dimensional reduction method for the scalar equation of (1.1).

To prove Theorem 1.1 we therefore work in strong topologies. We construct a blowing-up sequence of solutions $\left(u_{k}, T_{k}\right)_{k}$ of (1.1) whose scalar component writes as:

$$
u_{k}=u_{k, t, p}=W_{k, t, p}+u+\varphi_{k, t, p}
$$

but where this decomposition holds in $C^{0}(M)$ and not in $H^{1}(M)$. Here, as usual, $W_{k, t, p}$ denotes a bubbling profile depending on some parameters $(t, p) \in \mathbb{R}^{n+1}$ whose expression is given in Sections 2 and 7 below. The positive function $u$ is the scalar component of a solution $(u, T)$ of (1.1) satisfying a non-degeneracy assumption (detailed in (2.13) below). And the remainder term, $\varphi_{k, t, p}$, is chosen to be small with respect to $W_{k, t, p}$ and $u$ in the following pointwise sense:

$$
\left|\varphi_{k, t, p}\right| \leq \varepsilon_{k}\left(W_{k, t, p}+u\right) \quad \text { pointwise in } M
$$

for some suitably chosen sequence $\left(\varepsilon_{k}\right)_{k}$ of positive numbers converging to zero.

Decomposition (1.7) is of course reminiscent of Struwe's $H^{1}$ a priori result [4]. But the motivation for the choice of (1.7) comes from the work of Druet-Hebey-Robert [18, where Struwe's decomposition was shown to actually hold true in $C^{0}(M)$ for $H^{1}$-bounded solutions of critical stationary Schrödinger equations. The blow-up analysis of system (1.1) performed in 21] and 39] confirms the choice of (1.7) pointwise, at least at a local scale. 
The construction of a sequence $\left(u_{k}, T_{k}\right)_{k}$ satisfying (1.7) goes through an involved ping-pong procedure which relies upon a finite-dimensional reduction made possible by asymptotic analysis techniques. Section 2 introduces the definition of the coefficients $(h, f, \pi, \sigma, X, Y)$ and of the bubbling profiles considered and Section 9 gathers several technical results used throughout the article. The structure of the proof and the organization of the remaining sections of the article are as follows:

Section 3; Semi-decoupling and $H^{1}$ reduction. Let $\varphi$ be a remainder satisfying (1.8). Since $\vec{\triangle}_{g}$ has no Kernel by assumption, there exists a unique 1-form $T_{k, t, p}$ in $M$ satisfying $\vec{\triangle}_{g} T_{k, t, p}=$ $\left(W_{k, t, p}+u+\varphi\right)^{2^{*}} X+Y$. The $C^{0}$ bound (1.8) on $\varphi$ yields explicit pointwise bounds on $\mathcal{L}_{g} T_{k, t, p}$, which happens to blow-up too fast for standard $H^{1}$ finite-dimensional reduction procedures to apply to the scalar equation of (1.1) with $\mathcal{L}_{g} T_{k, t, p}$ seen as a coefficient. We therefore artificially discard the $\left|\mathcal{L}_{g} T_{k}+\sigma\right|_{g}^{2}$ term into a source term and consider instead the following equation:

$$
\triangle_{g} u+h u=f u^{2^{*}-1}+\frac{\left|\mathcal{L}_{g} T+\sigma\right|_{g}^{2}+\pi^{2}}{u^{2^{*}+1}} \underbrace{+\left(\frac{\left|\mathcal{L}_{g} T_{k}+\sigma\right|_{g}^{2}-\left|\mathcal{L}_{g} T+\sigma\right|_{g}^{2}}{\left(W_{k, t, p}+u+\varphi\right)^{2^{*}+1}}\right)}_{\text {Source term }},
$$

where $T$ satisfies $\vec{\triangle}_{g} T=u^{2^{*}} X+Y$. We perform a standard finite-dimensional reduction procedure and construct a solution of (1.9) - up to Kernel elements - having the form

$$
u_{k, t, p}=W_{k, t, p}+u+\psi,
$$

for some new remainder $\psi \in H^{1}(M)$.

Sections 4, 5] and 6: Pointwise asymptotic analysis of the remainder $\psi$ and fixedpoint argument. In view of the ping-pong argument, the main part of the proof is an involved application of Banach-Picard's fixed-point theorem to the remainders' mapping $\varphi \mapsto \psi \psi^{1}$ in the strong space of functions satisfying (1.8). The finite-dimensional reduction provides an $H^{1}$ bound on $\psi$ but says nothing about a $C^{0}$ control. In this part we show that $\psi$ still satisfies the pointwise estimate (1.8). A first step consists in showing that

$$
\psi=o\left(W_{k, t, p}+u\right) \text { in } C^{0}(M)
$$

as $k \rightarrow+\infty$. This step is achieved in Section 4 through an asymptotic blow-up analysis of the solution $u_{k, t, p}$ in (1.10) and relies on the machinery of the $C^{0}$ theory, developed in Druet-Hebey-Robert [18] (see also Hebey [25]), adapted to take into account the source term in (1.9).

A second step consists in quantifying the $o(1)$ in (1.11). This is the purpose of Section 5. Proposition 5.1 provides a local improvement of (1.11) in the region where the bubbling profile is dominant in the $C^{0}$ decomposition of $u_{k, t, p}$. It is obtained by a second-order blow-up analysis on and relies on the fact that, by construction, $\psi$ is orthogonal to the Kernel elements of the finite-dimensional reduction. The global improved version of (1.11) is Proposition 5.3. which yields the following control on $\psi$ : for some positive constant $C$ independent of $k, t, p$,

$$
|\psi| \leq C\left(\|X\|_{L^{\infty}} \varepsilon_{k}+\mu_{k}\right)\left(W_{k, t, p}+u\right) .
$$

The latter estimate implies in particular that, provided $\|X\|_{L^{\infty}(M)}$ is sufficiently small, $\psi$ satisfies again (1.8) for a suitable choice of $\left(\varepsilon_{k}\right)_{k}$.

Section 6 contains the last step in the proof of the fixed-point argument. The application of BanachPicard's fixed-point theorem is performed in Proposition 6.1 using again asymptotic analysis techniques in the spirit of those appearing in the proof of Proposition 5.1. In Proposition 6.3 we also

\footnotetext{
$1_{\text {we }}$ use the notations of the previous paragraph
} 
obtain an improvement of the global estimate on the remainder, in the region where the weak limit becomes dominant in the $C^{0}$ decomposition of $u_{k, t, p}$.

Sections 4 5 and 6 are the core of the analysis of the paper. The degree of precision of the local and global pointwise estimates on $\psi$ is also the key tool in the final step of the proof of Theorem 1.1.

\section{Sections 7 and 8;: Annihilation of the Kernel components.}

Section 6 provides us with a solution $\left(u_{k, t, p}, W_{k, t, p}\right)_{k}, u_{k, t, p}=W_{k, t, p}+u+\varphi_{k}(t, p)$, of (1.1) up to kernel elements of the scalar equation:

$$
\left\{\begin{array}{l}
\triangle_{g} u_{k, t, p}+h u_{k, t, p}=f u_{k, t, p}^{2^{*}-1}+\frac{\pi^{2}+\left|\sigma+\mathcal{L}_{g} W_{k, t, p}\right|_{g}^{2}}{u_{k, t, p}^{2^{*}+1}}+\sum_{j=0}^{n} \lambda_{k, j}(t, p) Z_{j, k, t, p}, \\
\vec{\triangle}_{g} W_{k, t, p}=u_{k, t, p}^{2^{*}} X+Y,
\end{array}\right.
$$

where the $Z_{j, k, t, p}$ are defined in (2.21) (see also Section 7). The proof is concluded by showing that one can annihilate all the $\lambda_{k, j}(t, p), 0 \leq j \leq n$, for a suitable value $\left(t_{k}, p_{k}\right)_{k}$ of the parameters.

In Section 7 we compute an asymptotic expansion of the $\lambda_{k, j}(t, p)$ as $k \rightarrow+\infty$. The lack of a variational structure forces us to again proceed differently than in the variational setting. Since the remainder $\varphi_{k}(t, p)$ of the solution $u_{k, t, p}$ was not obtained by variational means we only possess a rough estimate on $\left\|\varphi_{k}(t, p)\right\|_{H^{1}(M)}$. It is given by (6.2) below, and is intimately related to the choice of $\varepsilon_{k}$ - and hence to the (limited) precision of (5.56) below. In particular, no matter the precision on the choice of the ansatz $W_{k, t, p}+u$, we cannot hope to get a better estimate on $\left\|\varphi_{k}(t, p)\right\|_{H^{1}(M)}$. Therefore, the usual characterization of the $\lambda_{k, j}(t, p)$ in terms of a reduced energy does not hold here. We overcome this by estimating the $\lambda_{k, j}(t, p)$ directly, using the second-order pointwise estimates on $\varphi_{k}(t, p)$ and $\nabla \varphi_{k}(t, p)$ obtained in Sections 5 and 6. The conclusion of the proof of Theorem [1.1] is then given in Section 8, using a degree-theoretic argument.

Let us conclude this introduction with a few remarks:

- We prove Theorem 1.1 assuming that the $L^{\infty}$ norm of the coupling field $X$ is small, depending on $n, g, h, f, \pi, \sigma$ (see Section 22). This assumption is harmless, since smallness conditions on $X$ are necessary for solutions of (1.1) to exist: see [26, 37, 38.

- Our choice of the coefficients $(h, f, \pi, \sigma, X, Y)$ is driven by the a priori stability analysis of [39]. This is explicit in the choice of the order of smallness of $X$ at bumps, and in the localisation of the concentration point constructed. As shown in [39, the latter point is a common zero of $X$ and $\nabla f$ and a zero of $h-\frac{n-2}{4(n-1)} S_{g}$ (except in dimension 6 , see below).

- We chose to construct here non-compactness examples, which forces us to work with bubbling profiles supported in shrinking balls (see Section 2). Up to obvious modifications of the coefficients $(h, f, \pi, \sigma, X, Y)$, our proof works for bubbling profiles supported on balls of fixed positive radius thus yielding instability examples for (1.1).

- The scalar component of our blowing-up solutions is constructed to have a positive weak limit $u$. This is necessary, as shown by Lemma 3.1 below.

- The non-degeneracy assumption on $u$ is crucially used twice in our proof: to achieve the finite-dimensional reduction procedure on equation (1.9) and to prove Proposition [5.3. It does not restrain the choice of the coefficients $(h, f, \pi, \sigma, Y)$ : as shown in Section 2 , for small $\|X\|_{\infty}$, 1.11) always possesses such a non-degenerate solution.

- As already noticed in [40, the 6-dimensional case requires a more careful analysis. Roughly speaking, we are forced to perform an expansion of higher order than in dimensions $n \geq 7$, see Section 7 below. In particular, a rescaling of $X$ at a concentration point appears in the limiting expansion, see (7.44) and (7.45) below. 
Possible references for the finite-dimensional reduction method alluded to above, without pretending to be exhaustive, are Ambrosetti-Malchiodi 1], Berti-Malchiodi 4, Brendle 7], Brendle-Marques [8], Del Pino-Felmer-Musso [10] Del Pino-Musso-Pacard [11, Del Pino-Musso-Pacard-Pistoia [12, Rey [41, Rey-Wei [42, Robert-Vétois [44, 46, Wei 48, 49, 50, 51, and the references therein.

The a priori analysis techniques used in our proof have been developed in the context of the $C^{0}$ theory in Druet-Hebey-Robert [18, see also Druet [13], Druet-Hebey [17, Druet-Hebey-Vetois [20] and Hebey-Robert [27. Related techniques have independently been developed in the investigation of compactness phenomena for the Yamabe problem (see Li-Zhu [34, Druet [14, Marques [36, LiZhang [33, Khuri-Marques-Schoen [31]) and in the investigation of stability issues for nonlinear elliptic equations (Hebey-Wei [29], Druet-Hebey-Vétois [19]).

Acknowledgements: The author wants to thank O. Druet for fruitful discussions from which this paper originated.

\section{Setting of the Problem and notations}

In what follows we let $(M, g)$ be a closed $n$-dimensional Riemannian manifold, $n \geq 6$, of positive Yamabe type. We will always assume that $(M, g)$ possesses no non-trivial conformal killing 1-forms or, equivalently, that the operator $\vec{\triangle}_{g}$ is invertible in $M$. Let $\xi_{0} \in M$ and assume that $\left|W\left(\xi_{0}\right)\right|_{g}>0$ if $(M, g)$ is not locally conformally flat, where $W\left(\xi_{0}\right)$ denotes the Weyl tensor of $g$. The standard conformal normal coordinates result of Lee-Parker [32] asserts that there exists $\Lambda \in C^{\infty}(M \times M)$ such that for any point $\xi \in M$ there holds, for some arbitrarily large integer $N$ :

$$
\left|\left(\exp _{\xi}^{g_{\xi}}\right)^{*} g_{\xi}\right|(y)=1+O\left(|y|^{N}\right)
$$

$C^{1}$-uniformly in $\xi \in M$ and in $y \in T_{\xi} M$ in a small geodesic ball for the metric $g_{\xi}$. In (2.1) we have let

$$
g_{\xi}=\Lambda_{\xi}^{\frac{4}{n-2}} g
$$

where the conformal factor $\Lambda_{\xi}=\Lambda(\xi, \cdot)$ can in addition be chosen to satisfy:

$$
\Lambda_{\xi}(\xi)=1, \quad \nabla \Lambda_{\xi}(\xi)=0 .
$$

In (2.1), the notation $\exp _{\xi}^{g_{\xi}}$ denotes the exponential map for the metric $g_{\xi}$ at point $\xi$ with the identification of $T_{\xi} M$ to $\mathbb{R}^{n}$ via a smooth orthonormal basis $\left(e_{1}, \cdots, e_{n}\right)$ of $T_{\xi} M$ defined in a neighbourhood of $\xi_{0}$. The Lee-Parker [32] result also assert that there holds, for any $\xi \in M$ :

$$
S_{g_{\xi}}(\xi)=0, \quad \nabla S_{g_{\xi}}(\xi)=0, \quad \triangle_{g_{\xi}} S_{g_{\xi}}(\xi)=\frac{1}{6}\left|W_{g}(\xi)\right|_{g}^{2}
$$

where $S_{g_{\xi}}$ denotes the scalar curvature of the conformal metric $g_{\xi}$.

Let $\left(\tau_{k}\right)_{k}$ be a sequence of positive real numbers such that $\sum_{k} \tau_{k}<+\infty$. We define a sequence $\left(\mu_{k}\right)_{k}$ as follows:

$$
\mu_{k}=\left\{\begin{array}{lr}
\tau_{k} & \text { if } n=6, \\
\tau_{k}^{\frac{2}{n-6}} & \text { if }(M, g) \text { is l.c.f. or if } 7 \leq n \leq 10, \\
\tau_{k}^{\frac{1}{2}} & \text { if } n \geq 11 \text { and }(M, g) \text { is not l.c.f.. }
\end{array}\right.
$$

Let $\left(\xi_{k}\right)_{k}$ be a sequence of points of $M$ converging towards $\xi_{0}$ and satisfying $d_{g}\left(\xi_{k}, \xi_{k+1}\right)<<\frac{1}{k^{2}}$ as $k \rightarrow+\infty$. Let $\left(\beta_{k}\right)_{k}$ be a sequence of positive numbers converging to zero as $k \rightarrow+\infty$ and satisfying:

$$
\begin{array}{r}
\beta_{k}>>\mu_{k} \text { if } n \geq 7 \\
\mu_{k}^{\frac{1}{2}}>>\beta_{k}>>\mu_{k} \text { if } n=6 .
\end{array}
$$


Let $f$ be a smooth positive function, let $\sigma$ be a smooth traceless and divergence-free $(2,0)$-tensor in $M$ and let $\pi$ be a smooth function in $M$ with $\pi \not \equiv 0$. Let $Y$ be a smooth field of 1-forms and denote by $\tilde{Y}$ the only solution of $\vec{\triangle}_{g} \tilde{Y}=Y$ in $M$. We let also $H$ be a smooth nonnegative function in $\mathbb{R}^{n}$, compactly supported in $B_{0}(1)$ with $H(0)=1$, and for which 0 is a non-degenerate critical point.

The $n \geq 7$ case. We define:

$$
h=c_{n} S_{g}+\sum_{k \geq 0} \tau_{k} H\left(\frac{1}{\beta_{k}}\left(\exp _{\xi_{k}}^{g_{\xi_{k}}}\right)^{-1}(x)\right)
$$

where we have let $c_{n}=\frac{n-2}{4(n-1)}$ for all $n$. With this definition, $h$ is equal to $c_{n} S_{g}+\tau_{k} H\left(\frac{1}{\beta_{k}}\left(\exp _{\xi_{k}}^{g_{\xi_{k}}}\right)^{-1}(\cdot)\right)$ on every $B_{\xi_{k}}\left(\beta_{k}\right)$ - where the ball is taken for the metric $g_{\xi_{k}}$-and equals $c_{n} S_{g}$ outside of their reunion. Note, with (2.5) and (2.6) and since we are considering the $n \geq 7$ case here, that for any $r \in \mathbb{N}^{*}$, one can always choose $\beta_{k}$ as in (2.6) so that $h \in C^{r}(M)$. Let $u_{0}$ be a smooth, positive solution of the following Einstein-Lichnerowicz equation:

$$
\triangle_{g} u_{0}+h u_{0}=f u_{0}^{2^{*}-1}+\frac{\left|\mathcal{L}_{g} \tilde{Y}+\sigma\right|_{g}^{2}+\pi^{2}}{u_{0}^{2^{*}+1}},
$$

The coefficients $f, \pi, \sigma$ and $Y$ can always be chosen so that (2.8) possesses a smooth positive solution, see Hebey-Pacard-Pollack [26. Up to a slight modification of $f, \pi, \sigma, Y$ we can always assume $u_{0}$ to be strictly stable, that is satisfying, for any $\psi \in H^{1}(M)$ :

$$
\int_{M}|\nabla \psi|_{g}^{2}+\left[c_{n} S_{g}-\left(2^{*}-1\right) f u_{0}^{2^{*}-2}+\left(2^{*}+1\right) \frac{\left|\mathcal{L}_{g} \tilde{Y}+\sigma\right|_{g}^{2}+\pi^{2}}{u_{0}^{2^{*}+2}}\right] \psi^{2} d v_{g} \geq \frac{1}{C_{0}}\|\psi\|_{H^{1}}^{2},
$$

for some positive constant $C_{0}$. This is the case if $u_{0}$ is chosen to be the smallest solution of (2.8), see Premoselli [38, Section 7. Such a minimal solution is then the only strictly stable one of (2.8) (see Dupaigne [22], proposition 1.3.1).

The $n=6$ case. Up to assuming that the $\left(\tau_{k}\right)_{k}$ are small enough, and since $(M, g)$ is of positive Yamabe type, standard sub- and super-solution arguments show that there exists a unique positive solution $u_{0}$ of:

$$
\triangle_{g} u_{0}+\left(\frac{1}{5} S_{g}-\sum_{k \geq 0} \tau_{k} H\left(\frac{1}{\beta_{k}}\left(\exp _{\xi_{k}}^{g_{\xi_{k}}}\right)^{-1}(x)\right)\right) u_{0}=-f u_{0}^{2}+\frac{\left|\mathcal{L}_{g} \tilde{Y}+\sigma\right|_{g}^{2}+\pi^{2}}{u_{0}^{4}} .
$$

With (2.6), for any $0<\alpha<1$ such a $u_{0}$ can be chosen to belong to $C^{3, \alpha}(M)$ and it is then a positive and strictly stable solution (in the sense of (2.9) ) of the following Einstein-Lichnerowicz equation:

$$
\triangle_{g} u_{0}+h u_{0}=f u_{0}^{2}+\frac{\left|\mathcal{L}_{g} \tilde{Y}+\sigma\right|_{g}^{2}+\pi^{2}}{u_{0}^{4}}
$$

where we have let in this case:

$$
h=\frac{1}{5} S_{g}+2 f u_{0}-\sum_{k \geq 0} \tau_{k} H\left(\frac{1}{\beta_{k}}\left(\exp _{\xi_{k}}^{g_{\xi_{k}}}\right)^{-1}(x)\right) .
$$

Again, (2.5) and (2.6) ensure that this $h$ can be chosen to belong to $C^{1, \alpha}(M)$ for fixed $0<\alpha<1$, but not to $C^{2}(M)$, contrary to the $n \geq 7$ case.

Now, in every dimension $n \geq 6$, the implicit function theorem shows that there exists a constant $\eta_{0}=\eta_{0}(n, g, h, f, \pi, \sigma, Y)$ such that, for any field $X$ of 1-forms in $M$ satisfying

$$
\|X\|_{L_{\infty}(M)}=\eta \leq \eta_{0},
$$


the Einstein-Lichnerowicz system of equations:

$$
\left\{\begin{aligned}
\triangle_{g} u+h u & =f u^{2^{*}-1}+\frac{\left|\mathcal{L}_{g} T+\sigma\right|^{2}+\pi^{2}}{u^{2^{*}+1}} \\
\vec{\triangle}_{g} T & =u^{2^{*}} X+Y
\end{aligned}\right.
$$

possesses a solution $(u(X), T(X))$ such that $u(X) \rightarrow u_{0}$ in $C^{2}(M)$ as $\eta$, defined in (2.11), goes to 0 . In (2.12), $h$ is given by (2.7) or (2.10), depending on the dimension. Up to choosing $\eta$ small enough, it is easily seen that $u(X)$ is again a strictly stable solution of the scalar equation of (2.12), that is:

$$
\int_{M}|\nabla \psi|_{g}^{2}+\left[h-\left(2^{*}-1\right) f u(X)^{2^{*}-2}+\left(2^{*}+1\right) \frac{\left|\mathcal{L}_{g} T(X)+\sigma\right|_{g}^{2}+\pi^{2}}{u(X)^{2^{*}+2}}\right] \psi^{2} d v_{g} \geq \frac{1}{C}\|\psi\|_{H^{1}}^{2},
$$

for any $\psi \in H^{1}(M)$ and for some positive constant $C$ independent of $\eta$. In the following, for the sake of clarity and since $X$ will be fixed, the dependence on $X$ of the solutions $(u(X), T(X))$ will be omitted, and they will just be denoted by $(u, T)$.

The space $H^{1}(M)$ will denote the standard Sobolev space of functions in $L^{2}$ possessing $L^{2}$ distributional derivatives, and we endow $H^{1}(M)$ with the following scalar product: for any $u, v \in H^{1}(M)$,

$$
\langle u, v\rangle_{h}=\int_{M}\left(\langle\nabla u, \nabla v\rangle_{g}+h u v\right) d v_{g}
$$

where $h$ is given by (2.7) or (2.10). For any $J \in H^{1}(M)^{\prime}$ we will denote by $\left(\triangle_{g}+h\right)^{-1}(J)$ the unique element of $H^{1}(M)$ such that for any $v \in H^{1}(M)$ there holds:

$$
J(v)=\left\langle\left(\triangle_{g}+h\right)^{-1}(J), v\right\rangle_{h} .
$$

Since no ambiguity will occur, the notation $H^{1}(M)$ will also be used to denote the space of Sobolev 1-forms over $M$.

The blowing-up solutions of Theorem 1.1 are obtained by glueing degenerating peaks over the solution $(u, T)$. Here we define such peaks. Let $\left(r_{k}\right)_{k}$ be a sequence of positive real numbers which converges towards zero as $k \rightarrow \infty$ and satisfy:

$$
\beta_{k}<<r_{k}<<d_{g}\left(\xi_{k}, \xi_{k+1}\right) \quad \text { and } \quad r_{k}^{N}>>\mu_{k}
$$

where $\beta_{k}$ is given by (2.6), and for some large enough integer $N$, as $k \rightarrow+\infty$. The lowest value of $N$ required can be made explicit (only depending on $n$ ), as is easily seen in the proof of Theorem 1.1. Examples of sequences $\left(\tau_{k}\right)_{k},\left(\beta_{k}\right)_{k}$ and $\left(r_{k}\right)_{k}$ that satisfy (2.6), (2.15) and $\sum_{k} \tau_{k}<+\infty$ are easily found, for instance as different powers of $(1 / k)_{k}$. For $t>0$ we define the sequence $\left(\delta_{k}(t)\right)_{k}$ by:

$$
\delta_{k}(t)=\mu_{k} t
$$

where $\mu_{k}$ is as in (2.5). We let $r_{0}>0$ be such that $r_{0}<i_{g_{\xi}}(M)$ for all $\xi \in M$, where $i_{g_{\xi}}$ denotes the injectivity radius of the metric $g_{\xi}$ given by (2.2). By definition of $r_{k}$ there holds $2 r_{k}<r_{0}$ for any $k$. We let $\chi \in C^{\infty}(\mathbb{R})$ be a nonnegative, smooth compactly supported function such that $\chi \equiv 1$ in $[-1,1]$ and $\chi \equiv 0$ outside of $[-2,2]$. The blow-up profiles we investigate in this work are given by the following expression: for $t>0$ and $\xi \in M$, and for any $x \in M$ :

$$
W_{k, t, \xi}(x)=\Lambda_{\xi}(x) \chi\left(\frac{d_{g_{\xi}}(\xi, x)}{r_{k}}\right) \delta_{k}^{\frac{n-2}{2}}\left(\delta_{k}^{2}+\frac{f(\xi)}{n(n-2)} d_{g_{\xi}}(\xi, x)^{2}\right)^{1-\frac{n}{2}},
$$

where $\Lambda_{\xi}$ is as in (2.3) and $\delta_{k}$ is given by (2.16). These profiles are localized in $B_{\xi}\left(2 r_{k}\right)$, which denotes here the geodesic ball of radius $2 r_{k}$ with respect to the metric $g_{\xi}$. For a given $\xi \in M$ we let 
$V_{0, \xi}, \cdots, V_{n, \xi}: \mathbb{R}^{n} \rightarrow \mathbb{R}$ be given by:

$$
\begin{aligned}
& V_{0, \xi}(x)=\left(\frac{f(\xi)}{n(n-2)}|x|^{2}-1\right)\left(1+\frac{f(\xi)}{n(n-2)}|x|^{2}\right)^{-\frac{n}{2}} \\
& V_{i, \xi}(y)=f(\xi) x_{i}\left(1+\frac{f(\xi)}{n(n-2)}|x|^{2}\right)^{-\frac{n}{2}} .
\end{aligned}
$$

For any $0 \leq i \leq n$, the $V_{i, \xi}$ span the set of solutions in $H^{1}\left(\mathbb{R}^{n}\right)$ of the linearized equation (BianchiEgnell [5]):

where we have let:

$$
\triangle_{\text {eucl }} V_{i, \xi}=\left(2^{*}-1\right) f(\xi) U_{\xi}^{2^{*}-2} V_{i, \xi},
$$

$$
U_{\xi}(y)=\left(1+\frac{f(\xi)}{n(n-2)}|y|^{2}\right)^{1-\frac{n}{2}}, \text { for any } y \in \mathbb{R}^{n} .
$$

We also define, for any $x \in M$, any $1 \leq i \leq n$ and any $\xi \in M$ :

$$
\begin{aligned}
Z_{0, k, t, \xi}(x) & =\Lambda_{\xi}(x) \chi\left(\frac{d_{g_{\xi}}(\xi, x)}{r_{k}}\right) \delta_{k}^{\frac{n-2}{2}}\left(\delta_{k}^{2}+\frac{f(\xi)}{n(n-2)} d_{g_{\xi}}(\xi, x)^{2}\right)^{-\frac{n}{2}} \\
& \times\left(\frac{f(\xi)}{n(n-2)} d_{g_{\xi}}(\xi, x)^{2}-\delta_{k}^{2}\right) \\
Z_{i, k, t, \xi}(x) & =\Lambda_{\xi}(x) \chi\left(\frac{d_{g_{\xi}}(\xi, x)}{r_{k}}\right) \delta_{k}^{\frac{n}{2}}\left(\delta_{k}^{2}+\frac{f(\xi)}{n(n-2)} d_{g_{\xi}}(\xi, x)^{2}\right)^{-\frac{n}{2}} \\
& \times f(\xi)\left\langle\left(\exp _{\xi}^{g_{\xi}}\right)^{-1}(x), e_{i}(\xi)\right\rangle_{g_{\xi}(\xi)} .
\end{aligned}
$$

In (2.21), the $\left(e_{i}\right)_{i}$ denote the field of orthonormal basis centered at $\xi_{0}$ introduced in the beginning of this Section. Finally, we let

$$
K_{k, t, \xi}=\operatorname{Span}\left\{Z_{i, k, t, \xi}, i=0 \ldots n\right\} .
$$

Since $\left(V_{0, \xi}, \cdots, V_{n, \xi}\right)$ forms an orthonormal family for the scalar product $(u, v)=\int_{\mathbb{R}^{n}}\langle\nabla u, \nabla v\rangle d x$ in $\mathbb{R}^{n}, K_{k, t, \xi}$ is $(n+1)$-dimensional for $k$ large enough and the $Z_{i, k, t, \xi}$ are "almost" orthogonal. We denote by $K_{k, t, \xi}^{\perp}$ its orthogonal in $H^{1}(M)$ for the scalar product given by (2.14).

We did not specify the choice of $f$ and $X$ yet. Let $f_{0}>0$ be a positive constant. Here $f$ will be a perturbation of $f_{0}$ by small bumps:

$$
f=f_{0}+\sum_{k \geq 0} s_{k} \chi\left(\frac{1}{r_{k}}\left(\exp _{\xi_{k}}^{g_{\xi_{k}}}\right)^{-1}(x)\right),
$$

where $\left(s_{k}\right)_{k}$ is a sequence of real numbers converging to zero and satisfying $\left|s_{k}\right|=O\left(\mu_{k}^{N}\right)$ for a sufficiently large $N \in \mathbb{N}^{*}$. Let $X_{0}$ denote any smooth field of 1-forms in $M$ which vanishes in a neighbourhood of $\xi_{0}$. Let $Z$ be a fixed smooth 1-form in $\mathbb{R}^{n}$, compactly supported in $B_{0}(1)$, and with $\left|Z_{0}(0)\right|>0$. Define then, for any $x \in M$ :

$$
X(x)=X_{0}(x)+\sum_{k \geq 0} \mu_{k}^{\frac{n-1}{2}} Z\left(\frac{1}{r_{k}}\left(\exp _{\xi_{k}}^{g_{\xi_{k}}}\right)^{-1}(x)\right),
$$

where $\mu_{k}$ and $r_{k}$ are as in (2.5) and (2.15). Up to reducing $\left\|X_{0}\right\|_{\infty}$ and the $\tau_{k}$ such an $X$ always satisfies (2.11). In the following, we will also let:

$$
\alpha:=|Z(0)|=\frac{\left|X\left(\xi_{k}\right)\right|_{g_{\xi_{k}}}}{\mu_{k}^{\frac{n-1}{2}}} \text { for all } k .
$$


This parameter $\alpha$ will have to be chosen small, see the proof of Proposition 6.1. Again, with (2.5) and (2.15), $f$ and $X$ can always be chosen to belong to $C^{r}(M)$ for $r \in \mathbb{N}^{*}$.

Finally, let

$$
\mathcal{E}=\left\{\left(\varepsilon_{k}\right)_{k \in \mathbb{N}}, \varepsilon_{k}>0, \lim _{k \rightarrow \infty} \varepsilon_{k}=0\right\}
$$

be the set of sequences of positive real numbers converging to 0 . For $\left(\varepsilon_{k}\right)_{k} \in \mathcal{E}$ and for a given value of $(t, \xi) \in(0,+\infty) \times M$ we define the following sequence of subsets of $C^{2}(M)$ :

$$
F_{k}=F\left(\varepsilon_{k}, t, \xi\right)=\left\{v \in C^{0}(M) \text { such that }\left\|\frac{v}{u+W_{k, t, \xi}}\right\|_{C^{0}(M)} \leq \varepsilon_{k}\right\},
$$

where $u=u(X)$ is defined after (2.12) and $W_{k, t, \xi}$ is as in (2.17).

\section{An $H^{1}$ Finite-Dimensional REDUCTION METHOD FOR THE SEMI-DECOUPLED SYSTEM}

As discussed in the Introduction, the supercritical nonlinear coupling of system (1.1) makes the usual variational energy methods ineffective. In this Section we perform the first step of the fixed-point procedure in the proof of Theorem 1.1

As a first step in view of the $H^{1}$-theory, we get rid of the negative non-linearity in (1.1). We let, for any $\varepsilon>0$ and $r \in \mathbb{R}$ :

$$
\rho_{\varepsilon}(r)=\left\{\begin{array}{l}
\varepsilon \text { if } r<\varepsilon \\
r \text { if } r \geq \varepsilon .
\end{array}\right.
$$

We let $T$ denote any field of 1 -forms and we introduce the following truncation of the scalar equation of (1.1):

$$
\triangle_{g} u+h u=f u^{2^{*}-1}+\frac{\left|\mathcal{L}_{g} T+\sigma\right|^{2}+\pi^{2}}{\rho_{\varepsilon}(u)^{2^{*}+1}} .
$$

The following Lemma, proven in Premoselli-Wei [40, holds:

Lemma 3.1. There exists $\varepsilon_{0}>0$ depending only on $g, h, f, \pi$ such that for any 1-form $T$ and any $0 \leq \varepsilon \leq \varepsilon_{0}$, any $C^{2}$ positive solution $u$ of (3.1) satisfies:

$$
\min _{M} u \geq \varepsilon_{0} .
$$

In particular, for $0 \leq \varepsilon \leq \varepsilon_{0}$, any $C^{2}$ positive solution of (3.1) also solves:

$$
\triangle_{g} u+h u=f u^{2^{*}-1}+\frac{\left|\mathcal{L}_{g} T+\sigma\right|^{2}+\pi^{2}}{u^{2^{*}+1}} .
$$

In the following, if $\varepsilon_{0}$ is given by Lemma 3.1, we will let:

$$
\rho=\rho_{\varepsilon} \text { for some fixed } 0<\varepsilon \leq \frac{1}{4} \varepsilon_{0} .
$$

We now decouple system (1.1). Let $\left(\varepsilon_{k}\right)_{k} \in \mathcal{E},(t, \xi) \in(0,+\infty) \times M$ and $v_{k} \in F_{k}=F\left(\varepsilon_{k}, t, \xi\right)$, where $\mathcal{E}$ and $F_{k}$ are defined in (2.26) and (2.27). To every $v_{k}$ we associate a unique 1 -form $T_{k}=T_{k, t, \xi}$ defined as the unique solution in $M$ of

$$
\vec{\triangle}_{g} T_{k}=\left(u+W_{k, t, p}+v_{k}\right)^{2^{*}} X+Y
$$

Such a 1-form $T_{k}$ is unique since $(M, g)$ possesses no conformal Killing fields, and Proposition 9.2 provides us with sharp pointwise asymptotics on $T_{k}$. We introduce the following equation in $M$, which is the scalar equation of (1.1) with an additional source term, of unknown $w$ :

$$
\triangle_{g} w+h w=f w^{2^{*}-1}+\frac{\left|\mathcal{L}_{g} T+\sigma\right|^{2}+\pi^{2}}{\rho(w)^{2^{*}+1}}+\frac{\left|\mathcal{L}_{g} T_{k, t, \xi}+\sigma\right|_{g}^{2}-\left|\mathcal{L}_{g} T+\sigma\right|_{g}^{2}}{\left(u+W_{k, t_{k}, \xi_{k}}+v_{k}\right)^{2^{*}+1}},
$$


where $T_{k, t, \xi}$ is given by (3.3) and $(u, T)$ are as in (2.12). Choosing $v_{k}$ and investigating equation (3.4) amounts to semi-decoupling the system (1.1). In this section we solve equation (3.4) via a standard $H^{1}$ finite-dimensional reduction.

Classically, the first step consists in showing that the linearized operator for the scalar equation of (1.1) can be inverted in the orthogonal of $K_{k, t, \xi}$ :

Proposition 3.2. Let $D>0$. There exists a positive constant $C$ such that for any $(t, \xi) \in[1 / D, D] \times$ $M$ and for any $k$ there holds:

$$
\frac{1}{C}\|\psi\|_{H^{1}(M)} \leq\left\|L_{k, t, \xi}(\psi)\right\|_{H^{1}(M)} \leq C\|\psi\|_{H^{1}(M)} \text { for any } \psi \in K_{k, t, \xi},
$$

where $K_{k, t, \xi}$ is as in (2.22) and where we have let, for any $\psi \in K_{k, t, \xi}$ :

$$
\begin{array}{r}
L_{k, t, \xi}(\psi)=\Pi_{K_{k, t, \xi}^{\perp}}\left(\psi-\left(\triangle_{g}+h\right)^{-1}\left[\left(2^{*}-1\right) f\left(u+W_{k, t, \xi}\right)^{2^{*}-2} \psi\right.\right. \\
\left.\left.-\left(2^{*}+1\right) \frac{\left|\mathcal{L}_{g} T+\sigma\right|^{2}+\pi^{2}}{\left(u+W_{k, t, \xi}\right)^{2^{*}+2}} \psi\right]\right),
\end{array}
$$

where $\Pi_{K_{k, t, \xi}^{\perp}}$ denotes the orthogonal projection on $K_{k, t, \xi}^{\perp}$ with respect to the scalar product given in (2.14). In (3.6), $(u, T)=(u(X), T(X))$ are defined in the discussion following (2.12).

The proof of Proposition 3.2 follows from standard arguments and is clearly detailed in Robert-Vetois [45. The main result of this section is the resolution of the full non-linear equation (3.4), given by the following Proposition:

Proposition 3.3. Let $D>0$ and $\left(\varepsilon_{k}\right)_{k} \in \mathcal{E}$ and assume that $\varepsilon_{k}>>\mu_{k}^{\frac{3}{2}}$ as $k \rightarrow+\infty$, where $\mu_{k}$ is defined in (2.5). Let $\left(t_{k}, \xi_{k}\right)_{k}$ be a sequence in $[1 / D, D] \times M$ and, for any $k$, let $v_{k} \in F_{k}=F\left(\varepsilon_{k}, t_{k}, \xi_{k}\right)$. For $k$ large enough, there exists a function $\phi_{k}=\phi_{k}\left(t_{k}, \xi_{k}, v_{k}\right) \in K_{k, t_{k}, \xi_{k}}^{\perp}$ that solves the following equation:

$$
\begin{aligned}
& \Pi_{K_{k, t_{k}, \xi_{k}}^{\perp}}\left\{u+W_{k, t_{k}, \xi_{k}}+\phi_{k}\right. \\
& -\left(\triangle_{g}+h\right)^{-1}\left(f\left(u+W_{k, t_{k}, \xi_{k}}+\phi_{k}\right)^{2^{*}-1}+\frac{\left|\mathcal{L}_{g} T+\sigma\right|_{g}^{2}+\pi^{2}}{\rho\left(u+W_{k, t_{k}, \xi_{k}}+\phi_{k}\right)^{2^{*}+1}}\right) \\
& \left.-\left(\triangle_{g}+h\right)^{-1}\left(\frac{\left|\mathcal{L}_{g} T_{k}+\sigma\right|_{g}^{2}-\left|\mathcal{L}_{g} T+\sigma\right|_{g}^{2}}{\left(u+W_{k, t_{k}, \xi_{k}}+v_{k}\right)^{2^{*}+1}}\right)\right\}=0 .
\end{aligned}
$$

This $\phi_{k}$ is the unique solution of (3.7) in $K_{k, t_{k}, \xi_{k}}^{\perp} \cap B_{H^{1}(M)}\left(0, C \eta \varepsilon_{k}\right)$, where $C$ denotes some positive constant that does not depend on $k$, on the choice of $\left(t_{k}, \xi_{k}\right)_{k}$ or on $\eta$ as in (2.11). Also, in (3.7), $K_{k, t_{k}, \xi_{k}}$ is as in (2.22), $\rho$ is as in (3.2), and $T_{k}$ is as in (3.3).

As before, in (3.7) $(u, T)=(u(X), T(X))$ denote the specific solution of (2.12) obtained by the implicit function theorem when (2.11) holds. As an obvious consequence of Proposition 3.3, the function $\phi_{k}$ constructed therein satisfies:

$$
\phi_{k} \in K_{k, t_{k}, \xi_{k}}^{\perp} \text { for all }\left(t_{k}, \xi_{k}\right)_{k} \in[1 / D, D] \times M,
$$

and

$$
\left\|\phi_{k}\right\|_{H^{1}(M)} \leq C \eta \varepsilon_{k}
$$

for some constant $C$ which is independent of $\left(t_{k}, \xi_{k}\right)_{k}, k$ and $\eta$. 
Note that the necessity to introduce a source term in (3.4) is due to the blow-up behavior of $\mathcal{L}_{g} T_{k}$ as given by Proposition 9.2 below. Here, in the setting of Proposition 3.3, the finite-dimensional reduction will work since the nonlinearity $u \mapsto\left(\left|\mathcal{L}_{g} T+\sigma\right|^{2}+\pi^{2}\right) \rho(u)^{-2^{*}+1}$ is of subcritical type in the sense of Robert-Vétois [45]. But, as can be easily checked, there is no hope to even get an analogue of Proposition 3.2 if instead we considered $\left(\left|\mathcal{L}_{g} T_{k}+\sigma\right|^{2}+\pi^{2}\right) \rho(u)^{-2^{*}+1}$, with $T_{k}$ given by (3.3).

Proof. Let $D>0$ and $\left(\varepsilon_{k}\right)_{k} \in \mathcal{E}$. Assume that

$$
\varepsilon_{k}>>\mu_{k}^{\frac{3}{2}}
$$

where $\mu_{k}$ is as in (2.5). Let $\left(t_{k}, \xi_{k}\right)_{k} \in[1 / D, D] \times M$ and $\left(v_{k}\right)_{k}, v_{k} \in F_{k}=F_{\varepsilon_{k}, t_{k}, \xi_{k}}$ be fixed. In the proof of Proposition 3.3. for the sake of clarity and since no ambiguity will occur, we will omit the dependence in $t_{k}$ and $\xi_{k}$ in the quantities appearing. Hence we shall denote $K_{k, t_{k}, \xi_{k}}$ by $K_{k}, W_{k, t_{k}, \xi_{k}}$ by $W_{k}$ and so on. Let $\phi \in K_{k}^{\perp}$ be fixed. It is easily seen that $\phi$ solves:

$$
\begin{aligned}
\Pi_{K_{k}^{+}} & \left\{u+W_{k}+\phi\right. \\
& -\left(\triangle_{g}+h\right)^{-1}\left(f\left(u+W_{k}+\phi\right)^{2^{*}-1}+\frac{\left|\mathcal{L}_{g} T+\sigma\right|_{g}^{2}+\pi^{2}}{\rho\left(u+W_{k}+\phi\right)^{2^{*}+1}}\right) \\
& \left.+\left(\triangle_{g}+h\right)^{-1}\left(\frac{\left|\mathcal{L}_{g} T_{k}+\sigma\right|_{g}^{2}-\left|\mathcal{L}_{g} T+\sigma\right|_{g}^{2}}{\left(u+W_{k}+v_{k}\right)^{2^{*}+1}}\right)\right\}=0
\end{aligned}
$$

if and only if $\phi$ solves the fixed-point equation:

$$
\phi=\Theta_{k}(\phi) \quad \text { in } K_{k}^{\perp},
$$

where we have let

$$
\Theta_{k}(\phi)=L_{k}^{-1} \circ \Pi_{K_{k}^{\perp}} \circ\left(\triangle_{g}+h\right)^{-1} \circ N_{k}(\phi)-L_{k}^{-1} \circ \Pi_{K_{k}^{\perp}}\left(R_{k}\right),
$$

where $L_{k}=L_{k, t_{k}, \xi_{k}}$ is as in (3.6) and where in (3.11) we have let

$$
\begin{aligned}
N_{k}(\phi) & =f\left[\left(u+W_{k}+\phi\right)^{2^{*}-1}-\left(u+W_{k}\right)^{2^{*}-1}-\left(2^{*}-1\right)\left(u+W_{k}\right)^{2^{*}-2} \phi\right] \\
& +\left(\left|\mathcal{L}_{g} T+\sigma\right|_{g}^{2}+\pi^{2}\right)\left[\rho\left(u+W_{k}+\phi\right)^{-2^{*}-1}-\left(u+W_{k}\right)^{-2^{*}-1}+\left(2^{*}+1\right)\left(u+W_{k}\right)^{-2^{*}-2} \phi\right]
\end{aligned}
$$

and

$$
\begin{aligned}
R_{k}=u+W_{k}-\left(\triangle_{g}+h\right)^{-1} & \left(f\left(u+W_{k}\right)^{2^{*}-1}+\frac{\left|\mathcal{L}_{g} T+\sigma\right|_{g}^{2}+\pi^{2}}{\left(u+W_{k}\right)^{2^{*}+1}}\right) \\
& +\left(\triangle_{g}+h\right)^{-1}\left(\frac{\left|\mathcal{L}_{g} T_{k}+\sigma\right|_{g}^{2}-\left|\mathcal{L}_{g} T+\sigma\right|_{g}^{2}}{\left(u+W_{k}+v_{k}\right)^{2^{*}+1}}\right)
\end{aligned}
$$

Note that $\Theta_{k}$ is well-defined because of Proposition 3.2. We now apply Banach-Picard's fixed-point theorem to $\Theta_{k}$ defined in (3.11). Let $\phi_{1}, \phi_{2} \in K_{k}^{\perp}$. Standard computations show that there holds, for some positive constant $C$ independent of $k$ and $\eta$ as in (2.11):

$$
\begin{array}{r}
\left\|f\left[\left(u+W_{k}+\phi_{1}\right)^{2^{*}-1}-\left(u+W_{k}+\phi_{2}\right)^{2^{*}-1}-\left(2^{*}-1\right)\left(u+W_{k}\right)^{2^{*}-2}\left(\phi_{1}-\phi_{2}\right)\right]\right\|_{\left(H^{1}(M)\right)^{\prime}} \\
\leq C\left(\left\|\phi_{1}\right\|_{H^{1}(M)}^{\frac{4}{n-2}}+\left\|\phi_{2}\right\|_{H^{1}(M)}^{\frac{4}{n-2}}\right)\left\|\phi_{1}-\phi_{2}\right\|_{H^{1}(M)}
\end{array}
$$


and

$$
\begin{aligned}
& \|\left(\left|\mathcal{L}_{g} T+\sigma\right|_{g}^{2}+\pi^{2}\right)\left[\rho\left(u+W_{k}+\phi_{1}\right)^{-2^{*}-1}-\rho\left(u+W_{k}+\phi_{2}\right)^{-2^{*}-1}\right. \\
& \left.+\left(2^{*}+1\right)\left(u+W_{k}\right)^{-2^{*}-2}\left(\phi_{1}-\phi_{2}\right)\right]\|\|_{\left(H^{1}(M)\right)^{\prime}} \\
& \leq C \sup _{v \in\left[\phi_{1}, \phi_{2}\right]}\left\|\frac{\rho^{\prime}\left(u+W_{k}+v\right)}{\rho\left(u+W_{k}+v\right)^{2^{*}+2}}-\frac{1}{\left(u+W_{k}\right)^{2^{*}+2}}\right\|_{L^{\frac{n}{2}}(M)}\left\|\phi_{1}-\phi_{2}\right\|_{H^{1}(M)} .
\end{aligned}
$$

Using the definition of $\rho$ in (3.2) one obtains that for any $v \in\left[\phi_{1}, \phi_{2}\right] \subset K_{k}^{\perp}$ :

$$
\left\|\frac{\rho^{\prime}\left(u+W_{k}+v\right)}{\rho\left(u+W_{k}+v\right)^{2^{*}+2}}-\frac{1}{\left(u+W_{k}\right)^{2^{*}+2}}\right\|_{L^{\frac{n}{2}(M)}} \leq C\|v\|_{H^{1}(M)}^{\frac{4}{n-2}}
$$

where $C$ is a positive constant that does not depend on $k, \eta$ or $v \in\left[\phi_{1}, \phi_{2}\right]$. Gathering (3.14), (3.15) and (3.16) one therefore has that:

$$
\left\|\Theta_{k}\left(\phi_{1}\right)-\Theta_{k}\left(\phi_{2}\right)\right\|_{H^{1}(M)} \leq C_{1}\left(\left\|\phi_{1}\right\|_{H^{1}(M)}^{\frac{4}{n-2}}+\left\|\phi_{2}\right\|_{H^{1}(M)}^{\frac{4}{n-2}}\right)\left\|\phi_{1}-\phi_{2}\right\|_{H^{1}(M)}
$$

for some positive constant $C_{1}$ that neither depends on $k$ nor on $\eta$. We now estimate $\Theta_{k}(0)$. By Proposition 3.2 there holds:

$$
\left\|\Theta_{k}(0)\right\|_{H^{1}(M)} \leq C\left\|\left(\triangle_{g}+h\right) R_{k}\right\|_{L^{\frac{2 n}{n+2}}(M)},
$$

for some positive $C$ independent of $k$ and $\eta$, where $R_{k}$ is defined in (3.13). Straightforward computations using (2.7), (2.10) and (2.23) show that:

$$
\left\|\left(\triangle_{g}+h\right)\left(u+W_{k}\right)-f\left(u+W_{k}\right)^{2^{*}-1}+\frac{\left|\mathcal{L}_{g} T+\sigma\right|_{g}^{2}+\pi^{2}}{\left(u+W_{k}\right)^{2 *+1}}\right\|_{L^{\frac{2 n}{n+2}}(M)}=o\left(\delta_{k}^{\frac{3}{2}}\right)
$$

as $k \rightarrow+\infty$, and the $o\left(\delta_{k}^{\frac{3}{2}}\right)$ term in (3.19) is uniform in the choice of $\left(t_{k}, \xi_{k}\right)_{k} \in[1 / D, D] \times M$. We now write that

$$
\left|\mathcal{L}_{g} T_{k}+\sigma\right|_{g}^{2}-\left|\mathcal{L}_{g} T+\sigma\right|_{g}^{2}=\left\langle\mathcal{L}_{g} T_{k}-\mathcal{L}_{g} T, \mathcal{L}_{g} T_{k}+\mathcal{L}_{g} T+2 \sigma\right\rangle_{g}
$$

where the scalar product $\langle\cdot, \cdot\rangle_{g}$ is the standard one induced by the metric $g$ on $(2,0)$-tensors. Using the pointwise estimates on $\left|\mathcal{L}_{g} T_{k}-\mathcal{L}_{g} T\right|_{g}$ given by (9.11), (2.24) and (2.15) one finds that:

$$
\left\|\frac{\left|\mathcal{L}_{g} T_{k}+\sigma\right|_{g}^{2}-\left|\mathcal{L}_{g} T+\sigma\right|_{g}^{2}}{\left(u+W_{k}+v_{k}\right)^{2^{*}+1}}\right\|_{L^{\frac{2 n}{n+2}}(M)} \leq C^{\prime}\left(\eta \varepsilon_{k}+\delta_{k}^{\frac{n}{4}}\right)
$$

for some positive $C^{\prime}$ independent of $k$ and $\eta$. Remember that throughout this paper we always assume that $n \geq 6$, so (3.18), (3.19) and (3.20) show that there exists a positive constant $C_{2}$ independent of $k$ and $\eta$ such that:

$$
\left\|\Theta_{k}(0)\right\|_{H^{1}(M)} \leq C_{2}\left(\eta \varepsilon_{k}+\delta_{k}^{\frac{3}{2}}\right) .
$$

We let now

$$
c_{k}=2 C_{2} \eta \varepsilon_{k}
$$


where $C_{2}$ is given by (3.21) and $\eta$ is as in (2.11). With (3.17), (3.21) and (3.10) we obtain that, for any $\phi_{1}, \phi_{2} \in K_{k}^{\perp} \cap B_{H^{1}(M)}\left(0, c_{k}\right)$, there holds:

$$
\left\|\Theta_{k}\left(\phi_{1}\right)-\Theta_{k}\left(\phi_{2}\right)\right\|_{H^{1}(M)} \leq C_{1}\left(2 c_{k}^{\frac{4}{n-2}}\right)\left\|\phi_{1}-\phi_{2}\right\|_{H^{1}(M)}
$$

and

$$
\left\|\Theta_{k}\left(\phi_{1}\right)\right\|_{H^{1}(M)} \leq\left(\frac{1}{2}+o(1)\right) c_{k} .
$$

Up to choosing $k$ large enough, (3.23) and (3.24) show that $\Theta_{k}$ is $\frac{1}{2}$-Lipschitz from $B_{H^{1}(M)}\left(0,2 c_{k}\right)$ into itself. Banach-Picard's fixed-point theorem applies and, with (3.21), provides us with a function $\phi_{k} \in K_{k}^{\perp}$ satisfying (3.7), (3.8), (3.9), and which is the only solution of (3.7) in $K_{k} \cap B_{H^{1}(M)}\left(0, c_{k}\right)$, where $c_{k}$ is as in (3.22).

Let us point out again that the proof of Proposition 3.3 crucially relies on the pointwise estimates available on $v_{k}$, in particular for estimate (3.20).

\section{Pointwise $C^{0}$ estimates on $\phi_{k}$}

Let $D>0$ and $\left(\varepsilon_{k}\right)_{k} \in \mathcal{E}$, defined in (2.26). Assume that $\varepsilon_{k}>>\mu_{k}^{\frac{3}{2}}$ as $k \rightarrow+\infty$, where $\mu_{k}$ is defined in (2.5). Let $\left(t_{k}, \xi_{k}\right)_{k}$ be any sequence in $[1 / D, D] \times M$. For any $v_{k} \in F_{k}=F\left(\left(\varepsilon_{k}\right)_{k}, t_{k}, \xi_{k}\right)$, Proposition 3.3 shows the existence of a function $\phi_{k}=\phi_{k}\left(t_{k}, \xi_{k}, v_{k}\right) \in K_{k, t_{k}, \xi_{k}}^{\perp}$ that solves (3.7) and satisfies (3.8) and (3.9).

As discussed in the Introduction, the proof of Theorem 1.1 goes through the application of a BanachPicard fixed-point theorem to the mapping $v_{k} \mapsto \phi_{k}$. This requires to show in particular that such a mapping leaves $F_{k}$ defined in (2.27) invariant or, in other words, to obtain an explicit pointwise control on $\phi_{k}$. This task is achieved in this section and in the two following ones.

From here until the end of the paper, if $\left(f_{k}\right)_{k}$ denotes some sequence of real numbers or some sequence of functions, the notation $O\left(f_{k}\right)$ will denote a quantity whose absolute value can be bounded by the product of $\left|f_{k}\right|$ and of a constant independent of $k$ and $\eta$ as in (2.11). The notation $o\left(f_{k}\right)$ is defined accordingly. Similarly, we will write " $f_{k} \lesssim g_{k}$ " when there exists a positive constant $C$ independent of $k$ and $\eta$ as in (2.11) such that $f_{k} \leq C g_{k}$ for any $k$.

In this section we obtain a first, rough, asymptotic pointwise control of $\phi_{k}$ :

Proposition 4.1. Let $D>0$ and $\left(\varepsilon_{k}\right)_{k} \in \mathcal{E}$ and assume that $\varepsilon_{k}>>\mu_{k}^{\frac{3}{2}}$ as $k \rightarrow+\infty$, where $\mu_{k}$ is defined in (2.5). Let $\left(t_{k}, \xi_{k}\right)_{k}$ be a sequence of points in $[1 / D, D] \times M$, and let $v_{k} \in F_{k}=F\left(\varepsilon_{k}, t_{k}, \xi_{k}\right)$. There exists a sequence $\left(\nu_{k}\right)_{k}$ of positive numbers that goes to zero as $k \rightarrow+\infty$ such that:

$$
\left|\phi_{k}(x)\right| \leq \nu_{k}\left(u(x)+W_{k, t_{k}, \xi_{k}}(x)\right) \quad \text { for any } x \in M .
$$

In (4.1) we have let $\phi_{k}=\phi_{k}\left(t_{k}, \xi_{k}, v_{k}\right) \in K_{k, t_{k}, \xi_{k}}$ be the solution of (3.7) given by Proposition 3.3.

As a consequence of Proposition 4.1 Lemma 3.1 and Proposition 3.3 one has that for any $\left(t_{k}, \xi_{k}\right)_{k} \in$ $[1 / D, D] \times M$ and for any $v_{k} \in F\left(\varepsilon_{k}, t_{k}, \xi_{k}\right)$, letting

$$
u_{k, t_{k}, \xi_{k}, v_{k}}=u+W_{k, t_{k}, \xi_{k}}+\phi_{k}\left(t_{k}, \xi_{k}, v_{k}\right),
$$

there exist real numbers $\left(\lambda_{k}^{i}\left(t_{k}, \xi_{k}, v_{k}\right)\right)_{0 \leq i \leq n}$ such that $u_{k, t_{k}, \xi_{k}, v_{k}}$ satisfies:

$$
\begin{aligned}
&\left(\triangle_{g}+h\right) u_{k, t_{k}, \xi_{k}, v_{k}}-f u_{k, t_{k}, \xi_{k}, v_{k}}^{2^{*}-1}-\frac{\left|\mathcal{L}_{g} T+\sigma\right|_{g}^{2}+\pi^{2}}{u_{k, t_{k}, \xi_{k}, v_{k}}^{2^{*}}} \\
&-\frac{\left|\mathcal{L}_{g} T_{k}+\sigma\right|_{g}^{2}-\left|\mathcal{L}_{g} T+\sigma\right|_{g}^{2}}{\left(u+W_{k, t_{k}, \xi_{k}}+v_{k}\right)^{2^{*}+1}}=\sum_{i=0}^{n} \lambda_{k}^{i}\left(t_{k}, \xi_{k}, v_{k}\right)\left(\triangle_{g}+h\right) Z_{i, k} .
\end{aligned}
$$


Here again, $T_{k, t_{k}, \xi_{k}}$ is as in (3.3).

Proof. Let $D>0$ and $\left(\varepsilon_{k}\right)_{k} \in \mathcal{E}$ and assume that $\varepsilon_{k}>>\mu_{k}^{\frac{3}{2}}$ as $k \rightarrow+\infty$, where $\mu_{k}$ is defined in (2.5). Let $\left(t_{k}, \xi_{k}\right)_{k}$ be a sequence in $[1 / D, D] \times M$, let $v_{k} \in F_{k}=F\left(\varepsilon_{k}, t_{k}, \xi_{k}\right)$ and let $\phi_{k}=\phi_{k}\left(t_{k}, \xi_{k}, v_{k}\right) \in$ $K_{k, t_{k}, \xi_{k}}^{\perp}$ be the solution of (3.7) given by Proposition 3.3. In particular, for any $k$ large enough, there exist $\left(\lambda_{k}^{i}\right)_{0 \leq i \leq n}=\left(\lambda_{k}^{i}\left(t_{k}, \xi_{k}, v_{k}\right)\right)_{0 \leq i \leq n}$ such that $\phi_{k}$ solves:

$$
\begin{aligned}
\left(\triangle_{g}+h\right)\left(u+W_{k}+\phi_{k}\right) & -f\left(u+W_{k}+\phi_{k}\right)^{2^{*}-1}-\frac{\left|\mathcal{L}_{g} T+\sigma\right|_{g}^{2}+\pi^{2}}{\rho\left(u+W_{k}+\phi_{k}\right)^{2^{*}+1}} \\
& -\frac{\left|\mathcal{L}_{g} T_{k}+\sigma\right|_{g}^{2}-\left|\mathcal{L}_{g} T+\sigma\right|_{g}^{2}}{\left(u+W_{k}+v_{k}\right)^{2 *+1}}=\sum_{i=0}^{n} \lambda_{k}^{i}\left(\triangle_{g}+h\right) Z_{i, k},
\end{aligned}
$$

where the $Z_{i, k}$ are defined in (2.21) and where $T_{k}$ is as in (3.3). As before, in (4.4) and later on we shall omit the dependence in $t_{k}$ and $\xi_{k}$ and let $W_{k}=W_{k, t_{k}, \xi_{k}}$ and so on. A first, obvious remark is that (4.4) and standard elliptic regularity results show that $u+W_{k}+\phi_{k}$ belongs to $C^{1}(M)$, and then so does $\phi_{k}$. The proof of Proposition 4.1 goes through several steps.

Step 1: Estimation of the $\lambda_{k}^{i}$. We show that there holds:

$$
\sum_{i=0}^{n}\left|\lambda_{k}^{i}\right|=O\left(\eta \varepsilon_{k}\right)+O\left(\delta_{k}^{\frac{3}{2}}\right)
$$

For this, we rewrite (4.4) as:

$$
\begin{aligned}
\left(\triangle_{g}+h\right) R_{k}+ & \left(\triangle_{g}+h\right) \phi_{k}-f\left[\left(u+W_{k}+\phi_{k}\right)^{2^{*}-1}-\left(u+W_{k}\right)^{2^{*}-1}\right] \\
& -\left(\left|\mathcal{L}_{g} T+\sigma\right|_{g}^{2}+\pi^{2}\right)\left(\rho\left(u+W_{k}+\phi_{k}\right)^{-2^{*}-1}-\left(u+W_{k}\right)^{-2^{*}-1}\right) \\
& =\sum_{i=0}^{n} \lambda_{k}^{i}\left(\triangle_{g}+h\right) Z_{i, k},
\end{aligned}
$$

where $R_{k}$ is as in (3.13). Let $0 \leq i \leq n$ be fixed. There holds, by (3.9), (3.19), (3.20) and Hölder's inequality that:

$$
\left\langle R_{k}+\phi_{k}, Z_{i, k}\right\rangle_{h}=O\left(\delta_{k}^{\frac{3}{2}}\right)+O\left(\eta \varepsilon_{k}\right)
$$

Since we have:

$$
\left|\left(u+W_{k}+\phi_{k}\right)^{2^{*}-1}-\left(u+W_{k}\right)^{2^{*}-1}\right|=O\left(\left(u+W_{k}\right)^{2^{*}-2}\left|\phi_{k}\right|\right)+O\left(\left|\phi_{k}\right|^{2^{*}-1}\right),
$$

there holds that

$$
\int_{M} f\left[\left(u+W_{k}+\phi_{k}\right)^{2^{*}-1}-\left(u+W_{k}\right)^{2^{*}-1}\right] Z_{i, k} d v_{g}=O\left(\eta \varepsilon_{k}\right) .
$$

The sequence of functions $\left(\left(\left|\mathcal{L}_{g} T+\sigma\right|_{g}^{2}+\pi^{2}\right)\left(\rho\left(u+W_{k}+\phi_{k}\right)^{-2^{*}-1}-\left(u+W_{k}\right)^{-2^{*}-1}\right)\right)_{k}$ is uniformly bounded in $L^{\infty}(M)$, so we have, since $n \geq 6$ :

$$
\int_{M}\left(\left|\mathcal{L}_{g} T+\sigma\right|_{g}^{2}+\pi^{2}\right)\left(\rho\left(u+W_{k}+\phi_{k}\right)^{-2^{*}-1}-\left(u+W_{k}\right)^{-2^{*}-1}\right) Z_{i, k} d v_{g}=o\left(\delta_{k}^{\frac{3}{2}}\right) .
$$

Finally, there holds for any $0 \leq j \leq n$, that:

$$
\left\langle Z_{i, k}, Z_{j, k}\right\rangle_{h}=\delta_{i j}\left\|\nabla V_{i, \xi}\right\|_{L^{2}\left(\mathbb{R}^{n}\right)}^{2}+o(1)
$$

where $V_{i, \xi}$ is as in (2.18). Multiplying (4.6) by $Z_{i, k}$, integrating and using (4.7) - (4.10) yields (4.5). 
Step 2: Local behavior of $\phi_{k}$. In this step we show that:

$$
\delta_{k}^{\frac{n-2}{2}} u_{k}\left(\exp _{\xi_{k}}^{g_{\xi_{k}}}\left(\delta_{k} \cdot\right)\right) \longrightarrow U_{\xi_{0}} \text { in } C_{l o c}^{1}\left(\mathbb{R}^{n}\right),
$$

as $k \rightarrow+\infty$, where $\xi_{0}=\lim _{k \rightarrow+\infty} \xi_{k}, U_{\xi_{0}}$ is defined in (2.20), $\delta_{k}$ in (2.16) and the exponential map for $g_{\xi_{k}}$ is as in (2.1) and (2.2). In order to prove (4.11), we define $\tilde{u}_{k}$ in $B_{0}\left(r_{k} / \delta_{k}\right)$ by:

$$
\tilde{u}_{k}(x)=\delta_{k}^{\frac{n-2}{2}} u_{k}\left(\exp _{\xi_{k}}^{g_{\xi_{k}}}\left(\delta_{k} x\right)\right) .
$$

Remember that $r_{k}$ is a positive radius given by (2.15) and that $W_{k}$ is supported in $B_{\xi_{k}}\left(2 r_{k}\right)$, the ball being taken for the metric $g_{\xi_{k}}$. It is easily seen that for any $x \in B_{0}\left(r_{k} / \delta_{k}\right), \tilde{u}_{k}$ satisfies:

$$
\begin{aligned}
& \triangle_{g_{k}} \tilde{u}_{k}(x)+\delta_{k}^{2} h\left(x_{k}\right) \tilde{u}_{k}(x)=f\left(x_{k}\right) \tilde{u}_{k}(x)^{2^{*}-1}+\delta_{k}^{\frac{n+2}{2}} \frac{\left|\mathcal{L}_{g} T+\sigma\right|_{g}^{2}+\pi^{2}}{\rho\left(u+W_{k}+\phi_{k}\right)^{2^{*}+1}}\left(x_{k}\right) \\
& +\delta_{k}^{\frac{n+2}{2}} \frac{\left|\mathcal{L}_{g} T_{k}+\sigma\right|_{g}^{2}-\left|\mathcal{L}_{g} T+\sigma\right|_{g}^{2}}{\left(u+W_{k}+v_{k}\right)^{2^{*}+1}}\left(x_{k}\right)+\sum_{i=0}^{n} \lambda_{k}^{i} \delta_{k}^{\frac{n+2}{2}}\left(\triangle_{g}+h\right) Z_{i, k}\left(x_{k}\right),
\end{aligned}
$$

where, in (4.13), we have let $x_{k}=\exp _{\xi_{k}}^{g_{\xi_{k}}}\left(\delta_{k} x\right)$ and $g_{k}=\left(\exp _{\xi_{k}}^{g_{\xi_{k}}}\right)^{*} g_{\xi_{k}}\left(\delta_{k} \cdot\right)$. By definition of $\rho$ in (3.2) there holds:

$$
\delta_{k}^{\frac{n+2}{2}} \frac{\left|\mathcal{L}_{g} T+\sigma\right|_{g}^{2}+\pi^{2}}{\rho\left(u+W_{k}+\phi_{k}\right)^{2^{*}+1}}\left(x_{k}\right) \longrightarrow 0 \text { in } C_{l o c}^{0}\left(\mathbb{R}^{n}\right) .
$$

Using (9.11) below, (2.24), (2.15) and the pointwise control on $v_{k}$ given by the definition of $F_{k}$ in (2.27) we also have:

$$
\delta_{k}^{\frac{n+2}{2}} \frac{\left|\mathcal{L}_{g} T_{k}+\sigma\right|_{g}^{2}-\left|\mathcal{L}_{g} T+\sigma\right|_{g}^{2}}{\left(u+W_{k}+v_{k}\right)^{2^{*}+1}}\left(x_{k}\right) \longrightarrow 0 \text { in } C_{l o c}^{0}\left(\mathbb{R}^{n}\right)
$$

The Laplacian of $Z_{i, k}$ is computed using (9.2) below, and with (4.5) we also obtain that:

$$
\sum_{i=0}^{n} \lambda_{k}^{i} \delta_{k}^{\frac{n+2}{2}}\left(\triangle_{g}+h\right) Z_{i, k}\left(x_{k}\right) \longrightarrow 0 \text { in } L_{l o c}^{\infty}\left(\mathbb{R}^{n}\right) .
$$

By definition of $u_{k}$ in (4.2) and $\tilde{u}_{k}$ in (4.12) there holds, for any $x \in \mathbb{R}^{n}$, that:

$$
\lim _{r \rightarrow 0} \limsup _{k \rightarrow+\infty} \int_{B_{x}(r)} \tilde{u}_{k}^{2^{*}} d v_{g_{k}}=0
$$

and therefore an adaptation of Trudinger's standard argument (see for instance Hebey 25, theorem 2.15) along with (4.13), (4.14), (4.15) and (4.16) shows that $\tilde{u}_{k}$ converges strongly in $C_{l o c}^{1}\left(\mathbb{R}^{n}\right)$. By (3.9) and since $\varepsilon_{k} \rightarrow 0$, there holds

$$
\int_{K} \delta_{k}^{n}\left|\phi_{k}\left(\exp _{\xi_{k}}^{g_{\xi_{k}}}\left(\delta_{k} x\right)\right)\right|^{2^{*}} d v_{g_{k}}=\int_{\exp _{\xi_{k}}^{g_{\xi_{k}}\left(\delta_{k} K\right)}}\left|\phi_{k}\right|^{2^{*}} d v_{g}=o(1),
$$

so that using the explicit expression (4.2) of $u_{k}$, we see that (4.11) holds true.

Step 3: A lower-bound on $\phi_{k}$. We now show that for any sequence $\left(x_{k}\right)_{k}$ of points of $M$ there holds:

$$
\phi_{k}\left(x_{k}\right) \geq o\left(u\left(x_{k}\right)\right)+o\left(W_{k}\left(x_{k}\right)\right) .
$$


Let $G$ be the Green's function of the operator $\triangle_{g}+h$ in $M$ and let $\left(x_{k}\right)_{k}$ be a sequence of points in $M$. First, by the definition of $\rho$ in (3.2), since $\varepsilon_{k} \rightarrow 0$, by (3.9) and Fatou's lemma, we have:

$$
\begin{aligned}
& \int_{M} \frac{\left|\mathcal{L}_{g} T+\sigma\right|_{g}^{2}+\pi^{2}}{\rho\left(u+W_{k}+\phi_{k}\right)^{2^{*}+1}}(y) G\left(x_{k}, y\right) d v_{g}(y) \\
& \quad \geq \int_{M} \frac{\left|\mathcal{L}_{g} T+\sigma\right|_{g}^{2}+\pi^{2}}{u^{2^{*}+1}}(y) G\left(x_{k}, y\right) d v_{g}(y)+o(1)
\end{aligned}
$$

as $k \rightarrow+\infty$. Let $\left(A_{k}\right)_{k}$ denote some sequence of real numbers such that $A_{k} \rightarrow+\infty$ as $k \rightarrow \infty$ and such that $A_{k} \delta_{k} \rightarrow 0$ as $k \rightarrow \infty$. By (2.17) and (3.9),,$W_{k}+\phi_{k} \rightarrow 0$ almost everywhere in $M \backslash B_{x_{k}}\left(A_{k} \delta_{k}\right)$ and thus Fatou's lemma gives again that:

$$
\begin{aligned}
\int_{M \backslash B_{x_{k}}\left(A_{k} \delta_{k}\right)} f\left(u+W_{k}+\phi_{k}\right)^{2^{*}-1}(y) G\left(x_{k}, y\right) d v_{g}(y) \\
\geq \int_{M} f u^{2^{*}-1}(y) G\left(x_{k}, y\right) d v_{g}(y)+o(1) .
\end{aligned}
$$

Using (2.17), (3.9), the local convergence given in (4.11) and standard properties of Green's function (see e.g. Robert [43]), Fatou's lemma also shows that:

$$
\int_{B_{x_{k}}\left(A_{k} \delta_{k}\right)} f\left(u+W_{k}+\phi_{k}\right)^{2^{*}-1}(y) G\left(x_{k}, y\right) d v_{g}(y) \geq(1+o(1)) W_{k}\left(x_{k}\right)+o(1)
$$

as $k \rightarrow+\infty$ (see for instance Hebey [25], proposition 6.1). Independently, using the pointwise estimate given by (9.11) below, the definition of $X$ in (2.24), (2.15), the fact that $\varepsilon_{k} \rightarrow 0$ and standard properties of Green's function, one obtains that:

$$
\int_{M} \frac{\left|\mathcal{L}_{g} T_{k}+\sigma\right|_{g}^{2}-\left|\mathcal{L}_{g} T+\sigma\right|_{g}^{2}}{\left(u+W_{k}+v_{k}\right)^{2^{*}+1}}(y) G\left(x_{k}, y\right) d v_{g}(y)=o(1)
$$

as $k \rightarrow+\infty$. Having in mind that $\left|Z_{i, k}\right| \lesssim W_{k}$ for any $0 \leq i \leq n$, it remains to write a Green's representation formula for the operator $\triangle_{g}+h$ in $M$ and to use (4.4), (4.5), (4.18), (4.19), (4.20) and (4.21) to obtain that:

$$
\begin{array}{r}
u_{k}\left(x_{k}\right) \geq \int_{M} f u^{2^{*}-1}(y) G\left(x_{k}, y\right) d v_{g}(y)+\int_{M} \frac{\left|\mathcal{L}_{g} T+\sigma\right|_{g}^{2}+\pi^{2}}{u^{2^{*}+1}}(y) G\left(x_{k}, y\right) d v_{g}(y) \\
+(1+o(1)) W_{k}\left(x_{k}\right)+o(1) .
\end{array}
$$

Since $u$ solves the scalar equation of (2.12), a Green's representation formula for $u$ with (4.2) and (4.22) then concludes the proof of (4.17).

Note that, by the definition of $\rho$ in (3.2), (4.17) shows in particular that $u_{k}$ in (4.2) actually satisfies:

$$
\begin{aligned}
\left(\triangle_{g}+h\right) & u_{k}-f u_{k}^{2^{*}-1}-\frac{\left|\mathcal{L}_{g} T+\sigma\right|_{g}^{2}+\pi^{2}}{u_{k}^{2^{*}+1}} \\
& -\frac{\left|\mathcal{L}_{g} T_{k}+\sigma\right|_{g}^{2}-\left|\mathcal{L}_{g} T+\sigma\right|_{g}^{2}}{\left(u+W_{k}+v_{k}\right)^{2^{*}+1}}=\sum_{i=0}^{n} \lambda_{k}^{i}\left(\triangle_{g}+h\right) Z_{i, k} .
\end{aligned}
$$

Step 4: A global weak estimate on $\phi_{k}$. We prove now that

$$
\theta_{k}(x)^{\frac{n-2}{2}}\left|\phi_{k}(x)\right| \longrightarrow 0 \text { in } L^{\infty}(M)
$$

as $k \rightarrow+\infty$, where we have let, for any $x \in M$ :

$$
\theta_{k}(x)=\delta_{k}+d_{g_{\xi_{k}}}\left(\xi_{k}, x\right)
$$


The proof of (4.24) proceeds by contradiction: assume that there exists a sequence $\left(x_{k}\right)_{k}$ in $M$ such that

$$
\theta_{k}\left(x_{k}\right)^{2}\left|\phi_{k}\left(x_{k}\right)\right|^{2^{*}-2}=\max _{x \in M} \theta_{k}(x)^{2}\left|\phi_{k}(x)\right|^{2^{*}-2} \geq \varepsilon_{0}
$$

for some $\varepsilon_{0}>0$. We start by noticing that there holds:

$$
\theta_{k}\left(x_{k}\right)^{2} W_{k}\left(x_{k}\right)^{2^{*}-2}=o(1)
$$

and

$$
u_{k}\left(x_{k}\right) \rightarrow+\infty
$$

as $k \rightarrow+\infty$, where $x_{k}$ is defined in (4.24). Equations (4.27) and (4.28) follow from a straightforward adaptation of the arguments in Hebey [25] (proposition 7.1) combined with (4.5), (4.11), (4.17), (9.11) and (4.23). We let in what follows

$$
\tilde{\delta}_{k}=u_{k}\left(x_{k}\right)^{-\frac{2}{n-2}} .
$$

Equation (4.28) implies in particular that $\tilde{\delta}_{k} \rightarrow 0$ as $k \rightarrow+\infty$. For any $x \in B_{0}\left(i_{g}(M) / \tilde{\delta}_{k}\right)$, we let:

$$
\tilde{w}_{k}(x)=\tilde{\delta}_{k}^{\frac{n-2}{2}} u_{k}\left(\exp _{x_{k}}^{g_{x_{k}}}\left(\tilde{\delta}_{k} x\right)\right) \text {. }
$$

In case $\delta_{k}=o\left(\tilde{\delta}_{k}\right)$ and $d_{g_{x_{k}}}\left(x_{k}, \xi_{k}\right)=O\left(\tilde{\delta}_{k}\right)$, let $\mathcal{S}=\left\{\tilde{\xi}_{0}\right\}$, where $\tilde{\xi}_{0}=\lim _{k \rightarrow+\infty} \frac{1}{\delta_{k}}\left(\exp _{x_{k}}^{g_{x_{k}}}\right)^{-1}\left(\xi_{k}\right)$. Otherwise, let $\mathcal{S}=\emptyset$. Let $K \subset \subset \mathbb{R}^{n} \backslash \mathcal{S}$ be a compact set. For any $z \in K$, let $z_{k}=\exp _{x_{k}}^{g_{x_{k}}}\left(\tilde{\delta}_{k} x\right)$. There holds in particular that

$$
d_{g_{\xi_{k}}}\left(\xi_{k}, z_{k}\right) \geq C_{0} \tilde{\delta}_{k}
$$

for some positive constant $C_{0}$. Using (4.26), (4.27) and (4.29) one easily obtains that for any $x \in K$,

$$
\left|\tilde{w}_{k}(x)-\tilde{\delta}_{k}^{\frac{n-2}{2}} W_{k}\left(z_{k}\right)\right|^{2^{*}-2}=O(1),
$$

and the constant in the $O(1)$ term obviously depends on $K$. We now claim that

$$
\tilde{\delta}_{k}^{\frac{n-2}{2}} W_{k}\left(z_{k}\right)=o(1) \text {. }
$$

By (2.17) and (4.30), the only case where (4.32) is not clearly satisfied is when $\frac{1}{C} \delta_{k} \leq \tilde{\delta}_{k} \leq C \delta_{k}$ and $d_{g_{\xi_{k}}}\left(\xi_{k}, z_{k}\right) \leq C \delta_{k}$ for some positive constant $C$. In this case there also holds that $d_{g_{\xi_{k}}}\left(\xi_{k}, x_{k}\right)=$ $O\left(\delta_{k}\right)$, hence $\liminf \operatorname{in}_{k \rightarrow+\infty} \tilde{\delta}_{k}^{\frac{n-2}{2}} W_{k}\left(x_{k}\right)>0$ by definition of $W_{k}$, which contradicts (4.27). Hence (4.32) holds true. Coming back to (4.31) with (4.32) we have in particular:

$$
\tilde{w}_{k}(x) \leq C_{K} \text { for all } x \in K .
$$

By construction of $\tilde{w}_{k}$ in (4.29) there holds $\tilde{w}_{k}(0)=1$. As an easy consequence of (4.26) and of the definition of $\mathcal{S}$ above one always has $\left|\tilde{\xi}_{0}\right|>0$ whenever $\tilde{\xi}_{0}$ is finite. Hence (4.32) can be applied to some compact subset $K \ni 0$ and yields

$$
\tilde{\delta}_{k}^{\frac{n-2}{2}} W_{k}\left(x_{k}\right)=o(1)
$$

as $k \rightarrow+\infty$ which, combined with (4.26), also gives:

$$
\theta_{k}\left(x_{k}\right) \geq \frac{1}{C} \tilde{\delta}_{k}
$$


for some positive $C$ independent of $k$, where $\theta_{k}$ is as in (4.25). Let now $0 \in K \subset \subset \mathbb{R}^{n} \backslash \mathcal{S}$. By (4.23) and (4.29), $\tilde{w}_{k}$ satisfies, for any $x \in K$ :

$$
\begin{aligned}
\triangle_{\tilde{g}_{k}} \tilde{w}_{k}(x) & +\tilde{\delta}_{k}^{2} h\left(z_{k}\right) \tilde{w}_{k}(x)=f\left(z_{k}\right) \tilde{w}_{k}(x)^{2^{*}-1}+\tilde{\delta}_{k}^{2 n} \frac{\left(\left|\mathcal{L}_{g} T+\sigma\right|_{g}^{2}+\pi^{2}\right)\left(z_{k}\right)}{\tilde{w}_{k}^{2^{*}+1}(x)} \\
& +\tilde{\delta}_{k}^{\frac{n+2}{2}} \frac{\left|\mathcal{L}_{g} T_{k}+\sigma\right|_{g}^{2}-\left|\mathcal{L}_{g} T+\sigma\right|_{g}^{2}}{\left(u+W_{k}+v_{k}\right)^{2^{*}+1}}\left(z_{k}\right)+\tilde{\delta}_{k}^{\frac{n+2}{2}} \sum_{i=0}^{n} \lambda_{k}^{i}\left(\triangle_{g}+h\right) Z_{i, k}\left(z_{k}\right),
\end{aligned}
$$

where again $z_{k}=\exp _{x_{k}}^{g_{x_{k}}}\left(\tilde{\delta}_{k} x\right)$ and where $\tilde{g}_{k}=\left(\exp _{x_{k}}^{g_{x_{k}}}\right)^{*} g\left(\tilde{\delta}_{k} \cdot\right)$. Using estimate (9.11) below, (2.24) and using the pointwise estimates on $v_{k}$ given by the choice $v_{k} \in F_{k}=F\left(\varepsilon_{k}, t_{k}, \xi_{k}\right)$ it is easily seen that there holds

$$
\tilde{\delta}_{k}^{\frac{n+2}{2}} \frac{\left|\mathcal{L}_{g} T_{k}+\sigma\right|_{g}^{2}-\left|\mathcal{L}_{g} T+\sigma\right|_{g}^{2}}{\left(u+W_{k}+v_{k}\right)^{2^{*}+1}}\left(z_{k}\right)=O\left(\tilde{\delta}_{k}^{\frac{n+2}{2}}\right) \text { for any } x \in K .
$$

Combining (4.32) with the pointwise expression of $\left(\triangle_{h}+h\right) Z_{i, k}$ given by (9.2) below, the inequality $\left|Z_{i, k}\right| \lesssim W_{k}$ for $0 \leq i \leq n$ and with (2.15) yields:

$$
\tilde{\delta}_{k}^{\frac{n+2}{2}} \sum_{i=0}^{n} \lambda_{k}^{i}\left(\triangle_{g}+h\right) Z_{i, k}\left(z_{k}\right)=o(1) \text { uniformly in } K .
$$

In the end, (4.33), (4.37) and (4.38) give with (4.36) that $\tilde{w}_{k}$ satisfies in $K$ :

$$
\triangle_{\tilde{g}_{k}} \tilde{w}_{k}(x)+\tilde{\delta}_{k}^{2} h\left(z_{k}\right) \tilde{w}_{k}(x)=f\left(z_{k}\right) \tilde{w}_{k}(x)^{2^{*}-1}+\frac{o(1)}{\tilde{w}_{k}^{2^{*}+1}},
$$

where the term $o(1)$ is uniform in $K$. With (4.33) and (4.39), the Harnack inequality for the EinsteinLichnerowicz equation stated in Premoselli [39] (Proposition 6.1) then shows that there exists a positive constant $C_{K}$ such that

$$
\frac{1}{C_{K}} \leq \tilde{w}_{k} \leq C_{K} \text { in } K
$$

and thus, by standard elliptic theory, that $\tilde{w}_{k}$ converges in $C_{l o c}^{2}\left(\mathbb{R}^{n} \backslash \mathcal{S}\right)$ towards a positive solution $\tilde{w}_{0}$ of

$$
\triangle_{\xi} \tilde{w}_{0}=f\left(x_{0}\right) \tilde{w}_{0}^{2^{*}-1} \text { in } \mathbb{R}^{n} \backslash \mathcal{S},
$$

where $x_{0}=\lim _{k \rightarrow+\infty} x_{k}$. In particular, (4.40) shows that

$$
\int_{K} \tilde{w}_{0}^{2^{*}} d y>0 \text { for any compact set } K \subset \subset \mathbb{R}^{n} \backslash \mathcal{S} \text {. }
$$

But independently, by (4.29), (4.2), (4.32) and (3.9), one has that $\tilde{w}_{k} \rightarrow 0$ in $L^{2^{*}}(K)$ as $k \rightarrow+\infty$, which is a contradiction with (4.41). This concludes the proof of (4.24).

In the following we let, for any $\delta>0$ :

$$
\nu_{k}(\delta)=\sup _{M \backslash B_{\xi_{k}}(\delta)} u_{k} .
$$

Step 5: A first set of strong pointwise estimates. We show that for any $\varepsilon>0$, there exist $R_{\varepsilon}>0, \delta_{\varepsilon}>0$ and $C_{\varepsilon}>0$ such that:

$$
u_{k}(x) \leq C_{\varepsilon}\left(\delta_{k}^{\frac{n-2}{2}(1-2 \varepsilon)} d_{g_{\xi_{k}}}\left(\xi_{k}, x\right)^{(2-n)(1-\varepsilon)}+\nu_{k}\left(\delta_{\varepsilon}\right) d_{g_{\xi_{k}}}\left(\xi_{k}, x\right)^{(2-n) \varepsilon}\right),
$$

for any $k$ and for any $x \in M \backslash B_{\xi_{k}}\left(R_{\varepsilon} \delta_{k}\right)$. For $\varepsilon>0$, we define the following function in $M$ :

$$
\Psi_{k, \varepsilon}(x)=\delta_{k}^{\frac{n-2}{2}(1-2 \varepsilon)} G\left(\xi_{k}, x\right)^{1-\varepsilon}+\nu_{k}\left(\delta_{\varepsilon}\right) G\left(\xi_{k}, x\right)^{\varepsilon},
$$


where $G$ is the Green's function of $\triangle_{g}+h$ in $M$. We let $R>0$ and let $\left(x_{k}\right)_{k}$ be defined by:

$$
\frac{u_{k}}{\Psi_{k, \varepsilon}}\left(x_{k}\right)=\sup _{M \backslash B_{\xi_{k}}\left(R \delta_{k}\right)} \frac{u_{k}}{\Psi_{k, \varepsilon}} \text {. }
$$

We now claim that, up to choosing $R$ large enough and $\delta$ small enough, there holds, for $k>>1$, that:

$$
x_{k} \in \partial B_{\xi_{k}}\left(R \delta_{k}\right) \text { or } d_{g_{\xi_{k}}}\left(\xi_{k}, x_{k}\right) \geq \delta .
$$

The proof of (4.46) proceeds by contradiction: if we assume that (4.46) does not hold, then (4.45) gives that:

$$
d_{g_{\xi_{k}}}\left(\xi_{k}, x_{k}\right)^{2} \frac{\triangle_{g} \Psi_{k, \varepsilon}}{\Psi_{k, \varepsilon}}\left(x_{k}\right) \leq d_{g_{\xi_{k}}}\left(\xi_{k}, x_{k}\right)^{2} \frac{\triangle_{g} u_{k}}{u_{k}}\left(x_{k}\right) .
$$

Straightforward computations show that

$$
d_{g_{\xi_{k}}}\left(\xi_{k}, x_{k}\right)^{2} \frac{\triangle_{g} \Psi_{k, \varepsilon}}{\Psi_{k, \varepsilon}}\left(x_{k}\right) \geq-(1-\varepsilon)(1-C \varepsilon) d_{g_{\xi_{k}}}\left(\xi_{k}, x_{k}\right)^{2}+C \varepsilon(1-\varepsilon)
$$

for some positive constant $C$ independent of $k$. Independently, using (4.23) we have:

$$
\begin{aligned}
& d_{g_{\xi_{k}}}\left(\xi_{k}, x_{k}\right)^{2} \frac{\triangle_{g} u_{k}}{u_{k}}\left(x_{k}\right)=-d_{g_{\xi_{k}}}\left(\xi_{k}, x_{k}\right)^{2} h\left(x_{k}\right)+d_{g_{\xi_{k}}}\left(\xi_{k}, x_{k}\right)^{2} f\left(x_{k}\right) u_{k}^{2^{*}-2}\left(x_{k}\right) \\
& +d_{g_{\xi_{k}}}\left(\xi_{k}, x_{k}\right)^{2} \frac{\left|\mathcal{L}_{g} T+\sigma\right|_{g}^{2}+\pi^{2}}{u_{k}^{2^{*}+2}}\left(x_{k}\right)+d_{g_{\xi_{k}}}\left(\xi_{k}, x_{k}\right)^{2} \frac{\left|\mathcal{L}_{g} T_{k}+\sigma\right|_{g}^{2}-\left|\mathcal{L}_{g} T+\sigma\right|_{g}^{2}}{u_{k}\left(u+W_{k}+v_{k}\right)^{2^{*}+1}}\left(x_{k}\right) \\
& +d_{g_{\xi_{k}}}\left(\xi_{k}, x_{k}\right)^{2} \frac{1}{u_{k}} \sum_{i=0}^{n} \lambda_{k}^{i}\left(\triangle_{g}+h\right) Z_{i, k}\left(x_{k}\right) .
\end{aligned}
$$

By (4.2), (4.24) and since we assumed that (4.46) does not hold, we have that

$$
d_{g_{\xi_{k}}}\left(\xi_{k}, x_{k}\right)^{2} f\left(x_{k}\right) u_{k}^{2^{*}-2}\left(x_{k}\right) \leq o(1)+\|u\|_{L^{\infty}(M)}^{2^{*}-2} \delta^{2}+C\left(1+\frac{f\left(\xi_{k}\right)}{n(n-2)} R^{2}\right)^{-2}
$$

for some positive constant $C$ independent of $k$. Similarly, using in addition (4.17):

$$
d_{g_{\xi_{k}}}\left(\xi_{k}, x_{k}\right)^{2} \frac{\left|\mathcal{L}_{g} T+\sigma\right|_{g}^{2}+\pi^{2}}{u_{k}^{2^{*}+2}}\left(x_{k}\right) \leq C^{\prime} \delta^{2}
$$

for some positive $C^{\prime}$ independent of $k$. Here, $(u, T)$ are always as in (2.12). Using the pointwise estimates on $v_{k}$ given by (2.27), (2.24), (4.17), (2.15) and (9.11) below there holds:

$$
\begin{aligned}
d_{g_{\xi_{k}}}\left(\xi_{k}, x_{k}\right)^{2} & \frac{\left|\mathcal{L}_{g} T_{k}+\sigma\right|_{g}^{2}-\left|\mathcal{L}_{g} T+\sigma\right|_{g}^{2}}{u_{k}\left(u+W_{k}+v_{k}\right)^{2^{*}+1}} \\
\leq & \left\{\begin{array}{l}
O\left(\delta_{k}\right) \text { if } d_{g_{\xi_{k}}}\left(\xi_{k}, x_{k}\right) \leq \sqrt{\delta_{k}} \\
C\left(n, g, u_{0}\right) \delta^{2}+o(1) \text { if } d_{g_{\xi_{k}}}\left(\xi_{k}, x_{k}\right) \geq \sqrt{\delta_{k}} .
\end{array}\right.
\end{aligned}
$$

Finally, since $\left|Z_{i, k}\right| \lesssim W_{k}$, by (9.2) and (2.15) one gets, mimicking the proof of (4.50), that:

$$
\begin{aligned}
& d_{g_{\xi_{k}}}\left(\xi_{k}, x_{k}\right)^{2} \frac{1}{u_{k}} \sum_{i=0}^{n} \lambda_{k}^{i}\left(\triangle_{g}+h\right) Z_{i, k}\left(x_{k}\right) \\
& \quad \leq C\left(n, g, u_{0}, h\right) \delta^{2}+C\left(1+\frac{f\left(\xi_{k}\right)}{n(n-2)} R^{2}\right)^{-2}+o(1) .
\end{aligned}
$$

For a fixed value of $\varepsilon$, (4.47), (4.50), (4.51), (4.52) and (4.53) give a contradiction with (4.48) up to choosing $R$ large enough and $\delta$ small enough. This shows that (4.46) holds. Then (4.43) follows from (4.44) and the local convergence given by (4.11). 
Step 6: End of the proof of (4.1). We show that there exists a sequence $\nu_{k}$ of positive real numbers converging to 0 as $k \rightarrow+\infty$ such that for any $x \in M$ there holds:

$$
\left|\phi_{k}(x)\right| \leq \nu_{k}\left(W_{k}(x)+u(x)\right) .
$$

Let $\left(x_{k}\right)_{k}$ be a sequence of points in $M$. We prove in what follows that there holds:

$$
\left|\phi_{k}\left(x_{k}\right)\right|=o\left(W_{k}\left(x_{k}\right)\right)+o(1) \text {. }
$$

Since $u>0$ in $M$, (4.54) follows from (4.55). Assume first that $d_{g_{\xi_{k}}}\left(\xi_{k}, x_{k}\right)=O\left(\delta_{k}\right)$. Then (4.55) follows from (4.11). Assume then that $d_{g_{\xi_{k}}}\left(\xi_{k}, x_{k}\right) \not \rightarrow 0$ as $k \rightarrow+\infty$. Then (4.55) follows from (4.24). We may therefore assume that

$$
\delta_{k}<<d_{g_{\xi_{k}}}\left(\xi_{k}, x_{k}\right)<<1 .
$$

Let $G$ denote again the Green's function of $\triangle_{g}+h$. By (4.5) and since $\left|Z_{i, k}\left(x_{k}\right)\right| \lesssim W_{k}\left(x_{k}\right)$ for $0 \leq i \leq n$ we easily get that

$$
\int_{M} G\left(x_{k}, y\right) \sum_{i=0}^{n} \lambda_{k}^{i}\left(\triangle_{g}+h\right) Z_{i, k}(y) d v_{g}(y)=\sum_{i=0}^{n} \lambda_{k}^{i} Z_{i, k}\left(x_{k}\right)=o\left(W_{k}\left(x_{k}\right)\right) .
$$

Independently, since $\left(\left(\left|\mathcal{L}_{g} T+\sigma\right|_{g}^{2}+\pi^{2}\right) u_{k}^{-2^{*}-1}\right)_{k}$ is uniformly bounded in $L^{\infty}(M)$ by (4.17), we have that:

$$
\int_{M} G\left(x_{k}, y\right) \frac{\left|\mathcal{L}_{g} T+\sigma\right|_{g}^{2}+\pi^{2}}{u_{k}^{2^{*}+1}}(y) d v_{g}(y)=\int_{M} G\left(x_{k}, y\right) \frac{\left|\mathcal{L}_{g} T+\sigma\right|_{g}^{2}+\pi^{2}}{u^{2^{*}+1}}(y) d v_{g}(y)+o(1)
$$

as $k \rightarrow+\infty$. Now for some fixed $0<\varepsilon<\frac{2}{n+2}$ we write that

$$
\begin{aligned}
& \int_{M} G\left(x_{k}, y\right) f(y) u_{k}^{2^{*}-1}(y) d v_{g}(y) \\
& =\int_{B_{\xi_{k}}\left(R_{\varepsilon} \delta_{k}\right)} G\left(x_{k}, y\right) f(y) u_{k}^{2^{*}-1}(y) d v_{g}(y)+\int_{M \backslash B_{\xi_{k}}\left(R_{\varepsilon} \delta_{k}\right)} G\left(x_{k}, y\right) f(y) u_{k}^{2^{*}-1}(y) d v_{g}(y) .
\end{aligned}
$$

On the one side, (4.11) along with (4.56) shows that

$$
\int_{B_{\xi_{k}}\left(R_{\varepsilon} \delta_{k}\right)} G\left(x_{k}, y\right) f(y) u_{k}^{2^{*}-1}(y) d v_{g}(y)=O\left(W_{k}\left(x_{k}\right)\right)+o(1) .
$$

On the other side, using (4.43) we obtain that:

$$
\int_{M \backslash B_{\xi_{k}}\left(R_{\varepsilon} \delta_{k}\right)} G\left(x_{k}, y\right) f(y) u_{k}^{2^{*}-1}(y) d v_{g}(y)=O\left(W_{k}\left(x_{k}\right)\right)+O(1)
$$

as $k \rightarrow+\infty$. Gathering (4.21), (4.57), (4.58), (4.59) and (4.60) and writing a representation formula for $u_{k}$ gives, with (4.23), that :

$$
\left|\phi_{k}\left(x_{k}\right)\right| \leq C\left(W_{k}\left(x_{k}\right)+u\left(x_{k}\right)\right),
$$

for some positive constant $C$ that neither depends on $k$ nor on $\eta$ in (2.11). In particular, $C$ in (4.61) does not depend on the choice of $\left(t_{k}, \xi_{k}\right)_{k}$ and $\left(v_{k}\right)_{k}$. It remains to improve (4.61) into (4.55). Using the expression of the conformal laplacian of $W_{k}$ given by (9.1) below, and since $f \in C^{1}(M)$, the following representation formula holds true for $W_{k}$ :

$$
W_{k}\left(x_{k}\right)=\int_{M} G\left(x_{k}, y\right) f\left(\xi_{k}\right) W_{k}^{2^{*}-1}(y) d v_{g}(y)+o\left(W_{k}\left(x_{k}\right)\right)+o(1),
$$

where to obtain (4.62) we used that there holds, by (4.56) and Giraud's lemma (see 25], lemma 7.5):

$$
\int_{M} G\left(x_{k}, y\right) W_{k}(y) d v_{g}(y)=O\left(\delta_{k}^{\frac{n-2}{2}} \theta_{k}\left(x_{k}\right)^{4-n}\right)=O\left(\delta_{k} W_{k}\left(x_{k}\right)\right)+O\left(\delta_{k}\right),
$$


where $\theta_{k}$ is defined in (4.25). We now write a representation formula for $u_{k}-W_{k}-u$, where $u_{k}$ is defined in (4.2). Since $u$ solves (2.12), by (4.23), (4.57), (4.58), (4.21) and (4.62) there holds:

$$
\phi_{k}\left(x_{k}\right)=\int_{M} G\left(x_{k}, y\right) f(y)\left(u_{k}^{2^{*}-1}-W_{k}^{2^{*}-1}-u^{2^{*}-1}\right)(y) d v_{g}(y)+o\left(W_{k}\left(x_{k}\right)\right)+o(1) .
$$

By (4.11) there exists a sequence $A_{k} \rightarrow+\infty$ such that

$$
\left\|\delta_{k}^{\frac{n-2}{2}} u_{k}\left(\exp _{\xi_{k}}^{g_{\xi_{k}}}\left(\delta_{k} \cdot\right)\right)-U_{\xi_{0}}\right\|_{C^{0}\left(B_{0}\left(A_{k}\right)\right)} \rightarrow 0
$$

as $k \rightarrow+\infty$, where $U_{\xi_{0}}$ is defined in (2.20). We can always choose such a sequence $\left(A_{k}\right)_{k}$ to have $A_{k} \delta_{k}=o\left(\sqrt{\delta_{k}}\right)$, so that there holds, by the dominated convergence theorem:

$$
\int_{B_{\xi_{k}}\left(A_{k} \delta_{k}\right)} G\left(x_{k}, y\right) f(y)\left(u_{k}^{2^{*}-1}-W_{k}^{2^{*}-1}-u^{2^{*}-1}\right)(y) d v_{g}(y)=o\left(W_{k}\left(x_{k}\right)\right)+o(1) .
$$

By (2.17) and (4.61) there holds that

$$
\left|u_{k}^{2^{*}-1}-W_{k}^{2^{*}-1}-u^{2^{*}-1}\right| \lesssim W_{k}+\left|\phi_{k}\right| \text { in } M \backslash B_{\xi_{k}}\left(\sqrt{\delta_{k}}\right) .
$$

Since by (4.61) $\phi_{k}$ is uniformly bounded in $M \backslash B_{\xi_{k}}\left(\sqrt{\delta_{k}}\right)$ we obtain with (3.9), (4.63) and since $\varepsilon_{k}=o(1)$ that

$$
\int_{M \backslash B_{\xi_{k}}\left(\sqrt{\delta_{k}}\right)} G\left(x_{k}, y\right) f(y)\left(u_{k}^{2^{*}-1}-W_{k}^{2^{*}-1}-u^{2^{*}-1}\right)(y) d v_{g}(y)=o(1)+o\left(W_{k}\left(x_{k}\right)\right) .
$$

By (4.61) we can write that there holds in $B_{\xi_{k}}\left(\sqrt{\delta_{k}}\right)$ :

$$
\left|u_{k}^{2^{*}-1}-W_{k}^{2^{*}-1}-u^{2^{*}-1}\right| \lesssim W_{k}^{2^{*}-1} .
$$

Since $A_{k} \rightarrow+\infty$ as $k \rightarrow+\infty$ straightforward computations therefore show that:

$$
\int_{B_{\xi_{k}}\left(\sqrt{\delta_{k}}\right) \backslash B_{\xi_{k}}\left(A_{k} \delta_{k}\right)} G\left(x_{k}, y\right) f(y)\left(u_{k}^{2^{*}-1}-W_{k}^{2^{*}-1}-u^{2^{*}-1}\right)(y) d v_{g}(y)=o\left(W_{k}\left(x_{k}\right)\right)+o(1) .
$$

Equation (4.55) then follows from (4.64), (4.65), (4.66) and (4.67).

As a consequence of Proposition 4.1 we also obtain pointwise bounds on the gradient of $\phi_{k}$. More precisely there holds:

$$
\left|\nabla \phi_{k}(x)\right| \lesssim\left(1+\delta_{k}^{\frac{n-2}{2}} \theta_{k}(x)^{1-n}\right)
$$

where $\theta_{k}$ is defined in (4.25). Indeed, using Proposition 4.1, (2.24) and (9.11), equation (4.23) can be written as:

$$
\triangle_{g} \phi_{k}+h \phi_{k}=O\left(W_{k}^{2^{*}-1}\right)+O(1)
$$

so that writing again a representation formula for $\phi_{k}$ and differentiating yields easily (4.68).

\section{Second-Order estimates On $\phi_{k}$.}

Let $D>0$ and $\left(\varepsilon_{k}\right)_{k} \in \mathcal{E}$. Assume that $\varepsilon_{k}>>\mu_{k}^{\frac{3}{2}}$ as $k \rightarrow+\infty$, where $\mu_{k}$ is defined in (2.5). Let $\left(t_{k}, \xi_{k}\right)_{k} \in[1 / D, D] \times M$, for any $k$ let $v_{k} \in F_{k}=F\left(\left(\varepsilon_{k}\right)_{k}, t_{k}, \xi_{k}\right)$, and let $\phi_{k}=\phi_{k}\left(t_{k}, \xi_{k}, v_{k}\right)$ be given by Proposition 3.3

The asymptotic pointwise estimates on $\phi_{k}$ obtained in Proposition 4.1 are not accurate enough to even say that $\phi_{k}$ belongs to $F\left(\varepsilon_{k}, t, \xi\right)$. In view of the final fixed-point argument we need to control $\nu_{k}$ introduced in (4.1) only in terms of $\varepsilon_{k}$ and uniformly in the choice of $\left(t_{k}, \xi_{k}, v_{k}\right)_{k}$. This task is achieved in this section and in the following one. 
In this Section we perform a second-order pointwise expansion of $u_{k}$ defined in (4.2) and obtain finer pointwise estimates on $\phi_{k}$.

We keep using the notations $f_{k}=O\left(g_{k}\right), f_{k}=o\left(g_{k}\right)$ and $f_{k} \lesssim g_{k}$ introduced in Section 4 For the sake of clarity, we will also use the following notational shorthand: if $f \in L^{\infty}(M),\|f\|_{L^{\infty}\left(2 r_{k}\right)}$ will be used to denote the quantity $\|f\|_{L^{\infty}\left(B_{\xi_{k}}\left(2 r_{k}\right)\right)}$. Also, the notation $\mathbb{1}_{\text {nlc } f}$ will be used to denote a term which only appears when the manifold $M$ is non-locally conformally flat.

We first obtain refined estimates on $\phi_{k}$ when the bubble $W_{k}$ is the dominant term in the $C^{0}$ decomposition of $u_{k}$ :

Proposition 5.1. Let $D>0,\left(\varepsilon_{k}\right)_{k} \in \mathcal{E}$ and assume that $\varepsilon_{k}>>\mu_{k}^{\frac{3}{2}}$ as $k \rightarrow+\infty$, where $\mu_{k}$ is defined in (2.5). Let $\left(t_{k}, \xi_{k}\right)_{k}$ be a sequence in $[1 / D, D] \times M$, let $v_{k} \in F_{k}=F\left(\varepsilon_{k}, t_{k}, \xi_{k}\right)$ and let $\phi_{k}=\phi_{k}\left(t_{k}, \xi_{k}, v_{k}\right)$ be given by Proposition 3.3. Let $\left(x_{k}\right)_{k}$ be any sequence of points in $B_{\xi_{k}}\left(2 \sqrt{\delta_{k}}\right)$. There holds:

- If $n \geq 7$ :

$$
\begin{aligned}
\theta_{k}\left(x_{k}\right)\left|\nabla \phi_{k}\left(x_{k}\right)\right| & +\left|\phi_{k}\left(x_{k}\right)\right| \lesssim\left\|\phi_{k}\right\|_{L^{\infty}\left(\Omega_{k}\right)}+\sqrt{\delta_{k}}\left\|\nabla \phi_{k}\right\|_{L^{\infty}\left(\Omega_{k}\right)}+\delta_{k} \\
& +\left[\delta_{k}^{\frac{n}{2}}+\delta_{k}\|\nabla f\|_{L^{\infty}\left(2 r_{k}\right)}+\left\|h-c_{n} S_{g}\right\|_{L^{\infty}\left(2 r_{k}\right)} \delta_{k}^{2}\left|\ln \left(\frac{\theta_{k}\left(x_{k}\right)}{\delta_{k}}\right)\right|\right. \\
& \left.+\left\|h-c_{n} S_{g}\right\|_{L^{\infty}\left(2 r_{k}\right)} \theta_{k}\left(x_{k}\right)^{2}+\theta_{k}\left(x_{k}\right)^{4} \mathbb{1}_{n l c f}\right] W_{k}\left(x_{k}\right)+\left(\frac{\delta_{k}}{\theta_{k}\left(x_{k}\right)}\right)^{2} .
\end{aligned}
$$

- If $n=6$ :

$$
\begin{aligned}
& \theta_{k}\left(x_{k}\right)\left|\nabla \phi_{k}\left(x_{k}\right)\right|+\left|\phi_{k}\left(x_{k}\right)\right| \lesssim\left\|\phi_{k}\right\|_{L^{\infty}\left(\Omega_{k}\right)}+\sqrt{\delta_{k}}\left\|\nabla \phi_{k}\right\|_{L^{\infty}\left(\Omega_{k}\right)}+\delta_{k} \\
& +\left[\delta_{k}^{3}+\delta_{k}\|\nabla f\|_{L^{\infty}\left(2 r_{k}\right)}+\left\|h-\frac{1}{5} S_{g}-2 f u\right\|_{L^{\infty}\left(2 r_{k}\right)}\left(\delta_{k}^{2}\left|\ln \left(\frac{\theta_{k}\left(x_{k}\right)}{\delta_{k}}\right)\right|+\theta_{k}\left(x_{k}\right)^{2}\right)\right] W_{k}\left(x_{k}\right) .
\end{aligned}
$$

In (5.1) and (5.2) we have let:

$$
\Omega_{k}=B_{\xi_{k}}\left(2 r_{k}\right) \backslash B_{\xi_{k}}\left(\sqrt{\delta_{k}}\right)
$$

and $\theta_{k}\left(x_{k}\right)$ is as in (4.25).

Proof. Let $D>0$ and $\left(\varepsilon_{k}\right)_{k} \in \mathcal{E}$ and assume that $\varepsilon_{k}>>\mu_{k}^{\frac{3}{2}}$ as $k \rightarrow+\infty$, where $\mu_{k}$ is defined in (2.5). Let $\left(t_{k}, \xi_{k}\right)_{k}$ be a sequence in $[1 / D, D] \times M$, let $v_{k} \in F_{k}=F\left(\varepsilon_{k}, t_{k}, \xi_{k}\right)$ and let $\phi_{k}=\phi_{k}\left(t_{k}, \xi_{k}, v_{k}\right)$ be given by Proposition 3.3. Proposition 4.1 applies so that $u_{k}$ given by (4.2) solves (4.3). As before, we shall omit the dependence in $t_{k}$ and $\xi_{k}$ and let $W_{k}=W_{k, t_{k}, \xi_{k}}$ and so on. We first obtain pointwise estimates on $\phi_{k}$ that we later improve into gradient estimates.

We first assume that $n \geq 7$. From (4.3), using (2.12) and (9.1) below, $\phi_{k}$ is easily seen to satisfy:

$$
\begin{aligned}
\left(\triangle_{g}+h\right)\left(\phi_{k}-\sum_{i=0}^{n} \lambda_{k}^{i} Z_{i, k}\right) & =f\left(u_{k}^{2^{*}-1}-W_{k}^{2^{*}-1}-u^{2^{*}-1}\right) \\
& +\left(f-f\left(\xi_{k}\right)\right) W_{k}^{2^{*}-1}-\left(h-c_{n} S_{g}\right) W_{k} \\
& -c_{n} S_{g_{\xi_{k}}} \Lambda_{g_{\xi_{k}}}^{2^{*}} W_{k}+O\left(\delta_{k}^{\frac{n-2}{2}} \mathbb{1}_{d_{k} \leq 2 r_{k}}\right)+O\left(\delta_{k}^{\frac{n-2}{2}} r_{k}^{-n^{n}} \mathbb{1}_{r_{k} \leq d_{k} \leq 2 r_{k}}\right) \\
& +\left(\left|\mathcal{L}_{g} T+\sigma\right|_{g}^{2}+\pi^{2}\right)\left(u_{k}^{-2^{*}-1}-u^{-2^{*}-1}\right) \\
& +\frac{\left|\mathcal{L}_{g} T_{k}+\sigma\right|_{g}^{2}-\left|\mathcal{L}_{g} T+\sigma\right|_{g}^{2}}{\left(u+W_{k}+v_{k}\right)^{2^{*}+1}} .
\end{aligned}
$$


Remember that by (2.15) there holds that $r_{k}>>\sqrt{\delta_{k}}$ for $t \in[1 / D, D]$, where $\delta_{k}$ is given by (2.16). In (5.4) we have let, for any $x \in M$ :

$$
d_{k}(x)=d_{\xi_{\xi_{k}}}\left(\xi_{k}, x\right)
$$

Also, in (5.4), the notation $\delta_{k}^{\frac{n-2}{2}} r_{k}^{-n} \mathbb{1}_{r_{k} \leq d_{k} \leq 2 r_{k}}$ is used to denote a smooth function, supported in $B_{\xi_{k}}\left(2 r_{k}\right) \backslash B_{\xi_{k}}\left(r_{k}\right)$, and whose $C^{0}$ norm is bounded by $\delta_{k}^{\frac{n-2}{2}} r_{k}^{-n}$. Let $\left(x_{k}\right)_{k}$ be a sequence of points in $B_{\xi_{k}}\left(2 \sqrt{\delta_{k}}\right)$. If $x_{k} \in \Omega_{k}$ as in (5.3) we clearly have:

$$
\left|\phi_{k}\left(x_{k}\right)\right| \leq\left\|\phi_{k}\right\|_{L^{\infty}\left(\Omega_{k}\right)} .
$$

Assume now that $x_{k} \in B_{\xi_{k}}\left(\sqrt{\delta_{k}}\right)$. As before, we let $G$ denote the Green's function of $\triangle_{g}+h$ in $M$. A representation formula for $\phi_{k}-\sum_{i=0}^{n} \lambda_{k}^{i} Z_{i, k}$ in $B_{\xi_{k}}\left(2 \sqrt{\delta_{k}}\right)$ gives, using (2.15) and (5.4), that:

$$
\begin{aligned}
\phi_{k}\left(x_{k}\right) & =\sum_{i=0}^{n} \lambda_{k}^{i} Z_{i, k}\left(x_{k}\right)+O\left(\delta_{k}^{\frac{n-2}{2}} r_{k}^{2-n}\right)+O\left(\left\|\phi_{k}\right\|_{L^{\infty}\left(\Omega_{k}\right)}\right) \\
& +O\left(\sqrt{\delta_{k}}\left\|\nabla \phi_{k}\right\|_{L^{\infty}\left(\Omega_{k}\right)}\right)+O\left(\sum_{i=0}^{n}\left|\lambda_{k}^{i}\right|\right) \\
& +I_{1}+I_{2}+I_{3}+I_{4}+I_{5}+I_{6}
\end{aligned}
$$

where we have let:

$$
\begin{aligned}
& I_{1}=\int_{B_{\xi_{k}}\left(2 \sqrt{\delta_{k}}\right)} f(y)\left(u_{k}^{2^{*}-1}-W_{k}^{2^{*}-1}-u^{2^{*}-1}\right)(y) G\left(x_{k}, y\right) d v_{g}(y), \\
& I_{2}=\int_{B_{\xi_{k}}\left(2 \sqrt{\delta_{k}}\right)}\left(f(y)-f\left(\xi_{k}\right)\right) W_{k}^{2^{*}-1}(y) G\left(x_{k}, y\right) d v_{g}(y), \\
& I_{3}=-\int_{B_{\xi_{k}}\left(2 \sqrt{\delta_{k}}\right)}\left(h-c_{n} S_{g}\right)(y) W_{k}(y) G\left(x_{k}, y\right) d v_{g}(y), \\
& I_{4}=-\int_{B_{\xi_{k}}\left(2 \sqrt{\delta_{k}}\right)} c_{n} S_{\xi_{\xi_{k}}}(y) \Lambda_{g_{\xi_{k}}}^{2^{*}-2}(y) W_{k}(y) G\left(x_{k}, y\right) d v_{g}(y), \\
& I_{5}=\int_{B_{\xi_{k}}\left(2 \sqrt{\delta_{k}}\right)}\left(\left|\mathcal{L}_{g} T+\sigma\right|_{g}^{2}+\pi^{2}\right)\left(u_{k}^{-2^{*}-1}-u^{-2^{*}-1}\right)(y) G\left(x_{k}, y\right) d v_{g}(y), \text { and } \\
& I_{6}=\int_{B_{\xi_{k}}\left(2 \sqrt{\delta_{k}}\right)} \frac{\left|\mathcal{L}_{g} T_{k}+\sigma\right|_{g}^{2}-\left|\mathcal{L}_{g} T+\sigma\right|_{g}^{2}}{\left(u+W_{k}+v_{k}\right)^{2^{*}+1}}(y) G\left(x_{k}, y\right) d v_{g}(y) .
\end{aligned}
$$

The definition of $W_{k}$ in (2.17) yields:

$$
I_{2} \lesssim \delta_{k}\|\nabla f\|_{L^{\infty}\left(2 r_{k}\right)} W_{k}\left(x_{k}\right)
$$

while an application of Giraud's lemma (see [25], lemma 7.5) shows that

$$
I_{3} \lesssim\left\|h-c_{n} S_{g}\right\|_{L^{\infty}\left(2 r_{k}\right)} \theta_{k}\left(x_{k}\right)^{2} W_{k}\left(x_{k}\right)
$$

and, using (2.4), that:

$$
I_{4}=\left\{\begin{array}{lr}
0 & \text { if }(M, g) \text { is locally conformally flat } \\
O\left(\theta_{k}\left(x_{k}\right)^{4} W_{k}\left(x_{k}\right)\right) & \text { if } n \geq 7 \text { and }(M, g) \text { is not l.c.f. } \\
O\left(\delta_{k}^{2}\left|\ln \left(\theta_{k}\left(x_{k}\right)\right)\right|\right) & \text { if } n=6 \text { and }(M, g) \text { is not l.c.f }
\end{array}\right.
$$

where $\theta_{k}(x)$ is as in (4.25). Since $u_{k}$ is uniformly bounded from below by (4.17) we also have:

$$
I_{5} \lesssim \delta_{k}
$$


With (9.11) below and since $v_{k} \in F_{k}$ (defined in (2.27) $)$, we obtain that:

$$
I_{6} \lesssim \delta_{k} .
$$

Note that (5.9), (5.11), (5.12) and (5.13) actually hold even if $n=6$. By (4.1) there holds, in $B_{\xi_{k}}\left(\sqrt{\delta_{k}}\right)$ :

$$
\left|u_{k}^{2^{*}-1}-W_{k}^{2^{*}-1}-u^{2^{*}-1}\right| \lesssim W_{k}^{2^{*}-2}\left|\phi_{k}\right|+W_{k}^{2^{*}-2},
$$

so that we have

$$
I_{1} \lesssim\left(\frac{\delta_{k}}{\theta_{k}\left(x_{k}\right)}\right)^{2}\left(\left\|\phi_{k}\right\|_{L^{\infty}\left(B_{\xi_{k}}\left(2 \sqrt{\delta_{k}}\right)\right)}+1\right) .
$$

Gathering the estimates (5.9) to (5.15) in (5.7) and using (2.15) gives:

$$
\begin{aligned}
& \left|\phi_{k}\left(x_{k}\right)-\sum_{i=0}^{n} \lambda_{k}^{i} Z_{i, k}\left(x_{k}\right)\right| \lesssim\left\|\phi_{k}\right\|_{L^{\infty}\left(\Omega_{k}\right)}+\sqrt{\delta_{k}}\left\|\nabla \phi_{k}\right\|_{L^{\infty}\left(\Omega_{k}\right)}+\sum_{i=0}^{n}\left|\lambda_{k}^{i}\right| \\
& \quad+\delta_{k}+\left(\frac{\delta_{k}}{\theta_{k}\left(x_{k}\right)}\right)^{2}\left(1+\left\|\phi_{k}\right\|_{L^{\infty}\left(B_{\xi_{k}}\left(2 \sqrt{\delta_{k}}\right)\right)}\right) \\
& \quad+\left[\delta_{k}\|\nabla f\|_{L^{\infty}\left(2 r_{k}\right)}+\left\|h-c_{n} S_{g}\right\|_{L^{\infty}\left(2 r_{k}\right)} \theta_{k}\left(x_{k}\right)^{2}+\theta_{k}\left(x_{k}\right)^{4} \mathbb{1}_{n l c f}\right] W_{k}\left(x_{k}\right) .
\end{aligned}
$$

In (5.16), the notation $\mathbb{1}_{n l c f}$ is used to denote a term which vanishes when $(M, g)$ is conformally flat. Equation (5.16) holds for any sequence $x_{k} \in B_{\xi_{k}}\left(\sqrt{\delta_{k}}\right)$. Applying it to a well-chosen set of $(n+1)$ points lying in $B_{\xi_{k}}\left(\delta_{k}\right)$ one obtains that:

$$
\begin{aligned}
\sum_{i=0}^{n}\left|\lambda_{k}^{i}\right| & \lesssim \delta_{k}^{\frac{n-2}{2}}\left(\left\|\phi_{k}\right\|_{L^{\infty}\left(\Omega_{k}\right)}+\sqrt{\delta_{k}}\left\|\nabla \phi_{k}\right\|_{L^{\infty}\left(\Omega_{k}\right)}\right) \\
& +\delta_{k}^{\frac{n-2}{2}}+\delta_{k}\|\nabla f\|_{L^{\infty}\left(2 r_{k}\right)}+\left\|h-c_{n} S_{g}\right\|_{L^{\infty}\left(2 r_{k}\right)} \delta_{k}^{2} \\
& +\delta_{k}^{\frac{n-2}{2}}\left\|\phi_{k}\right\|_{L^{\infty}\left(B_{\xi_{k}}\left(2 \sqrt{\delta_{k}}\right)\right)}+\delta_{k}^{4} \mathbb{1}_{n l c f} .
\end{aligned}
$$

Plugging (5.17) into (5.16), using that $\left|Z_{i, k}\right| \lesssim W_{k}$ and using (5.6) gives the improved estimate:

$$
\begin{aligned}
\left|\phi_{k}\left(x_{k}\right)\right| & \lesssim\left\|\phi_{k}\right\|_{L^{\infty}\left(\Omega_{k}\right)}+\sqrt{\delta_{k}}\left\|\nabla \phi_{k}\right\|_{L^{\infty}\left(\Omega_{k}\right)} \\
& +\delta_{k}+\left(1+\left\|\phi_{k}\right\|_{L^{\infty}\left(B_{\xi_{k}}\left(2 \sqrt{\delta_{k}}\right)\right)}\right)\left(\frac{\delta_{k}}{\theta_{k}\left(x_{k}\right)}\right)^{2} \\
& +\left[\delta_{k}\|\nabla f\|_{L^{\infty}\left(2 r_{k}\right)}+\left\|h-c_{n} S_{g}\right\|_{L^{\infty}\left(2 r_{k}\right)} \theta_{k}\left(x_{k}\right)^{2}+\theta_{k}\left(x_{k}\right)^{4} \mathbb{1}_{n l c f}\right] W_{k}\left(x_{k}\right),
\end{aligned}
$$

which now holds for any sequence $x_{k} \in B_{\xi_{k}}\left(2 \sqrt{\delta_{k}}\right)$. We now claim that the following holds:

Claim 5.2. There holds

$$
\left\|\phi_{k}\right\|_{L^{\infty}\left(B_{\xi_{k}}\left(2 \sqrt{\delta_{k}}\right)\right)} \lesssim \max \left(1, M_{k}\right),
$$

where we have let

$$
\begin{aligned}
M_{k} & =\left\|\phi_{k}\right\|_{L^{\infty}\left(\Omega_{k}\right)}+\sqrt{\delta_{k}}\left\|\nabla \phi_{k}\right\|_{L^{\infty}\left(\Omega_{k}\right)}+\delta_{k} \\
& +\delta_{k}^{2-\frac{n}{2}}\|\nabla f\|_{L^{\infty}\left(2 r_{k}\right)}+\delta_{k}^{3-\frac{n}{2}}\left\|h-c_{n} S_{g}\right\|_{L^{\infty}\left(2 r_{k}\right)}+\delta_{k}^{5-\frac{n}{2}} \mathbb{1}_{n l c f},
\end{aligned}
$$

and where $\Omega_{k}$ is as in (5.3). 
Proof of Claim 5.2. Let $\left(x_{k}\right)_{k}$ be a sequence of points of $B_{\xi_{k}}\left(2 \sqrt{\delta_{k}}\right)$ satisfying $\left|\phi_{k}\left(x_{k}\right)\right|=\max _{B_{\xi_{k}}\left(2 \sqrt{\delta_{k}}\right)}\left|\phi_{k}\right|$. Claim 5.2 is trivially satisfied if $x_{k} \in \Omega_{k}$, so we assume in the following that $x_{k} \in B_{\xi_{k}}\left(\sqrt{\delta_{k}}\right)$. We proceed by contradiction and assume that there holds:

$$
\left|\phi_{k}\left(x_{k}\right)\right|=\left\|\phi_{k}\right\|_{L^{\infty}\left(B_{\xi_{k}}\left(2 \sqrt{\delta_{k}}\right)\right)}>>\max \left(1, M_{k}\right)
$$

as $k \rightarrow+\infty$, where $M_{k}$ is defined in (5.20). Let $\left(y_{k}\right)_{k}$ be any other sequence of points in $B_{\xi_{k}}\left(\sqrt{\delta_{k}}\right)$. Assumption (5.21) implies in particular that $\left\|\phi_{k}\right\|_{L^{\infty}\left(B_{\xi_{k}}\left(2 \sqrt{\delta_{k}}\right)\right)}>>1$, so that applying (5.18) at $y_{k}$ yields, with (5.21):

$$
\left|\phi_{k}\left(y_{k}\right)\right| \lesssim\left(\left(\frac{\delta_{k}}{\theta_{k}\left(y_{k}\right)}\right)^{2}+o(1)\right)\left\|\phi_{k}\right\|_{L^{\infty}\left(B_{\xi_{k}}\left(2 \sqrt{\delta_{k}}\right)\right)} .
$$

Using (5.22), we now compute again $I_{1}$ in (5.8) and obtain, since $\delta_{k} \leq \theta_{k}\left(y_{k}\right)$ :

$$
I_{1} \lesssim\left(\left(\frac{\delta_{k}}{\theta_{k}\left(y_{k}\right)}\right)^{\frac{7}{2}}+o(1)\right)\left\|\phi_{k}\right\|_{L^{\infty}\left(B_{\xi_{k}}\left(2 \sqrt{\delta_{k}}\right)\right)}+\left(\frac{\delta_{k}}{\theta_{k}\left(y_{k}\right)}\right)^{2},
$$

which in turn, with (5.7) and (5.21) implies that there holds:

$$
\left|\phi_{k}\left(y_{k}\right)\right| \lesssim\left(\left(\frac{\delta_{k}}{\theta_{k}\left(y_{k}\right)}\right)^{\frac{7}{2}}+o(1)\right)\left\|\phi_{k}\right\|_{L^{\infty}\left(B_{\xi_{k}}\left(2 \sqrt{\delta_{k}}\right)\right)} .
$$

Replugging the latter estimate in the computation of $I_{1}$ improves again, and after a finite number of iterations one obtains:

$$
\left|\phi_{k}\left(y_{k}\right)\right| \lesssim\left(\left(\frac{\delta_{k}}{\theta_{k}\left(y_{k}\right)}\right)^{n-2}+o(1)\right)\left\|\phi_{k}\right\|_{L^{\infty}\left(B_{\xi_{k}}\left(2 \sqrt{\delta_{k}}\right)\right)} \text { for any } y_{k} \in B_{\xi_{k}}\left(\sqrt{\delta_{k}}\right) .
$$

In particular, (5.23) applied to the sequence $x_{k}$ given by (5.21) yields:

$$
\theta_{k}\left(x_{k}\right) \lesssim \delta_{k}
$$

where $\theta_{k}$ is as in (4.25). We define now, for $y \in B_{0}\left(2 \delta_{k}^{-\frac{1}{2}}\right)$ :

$$
\tilde{\phi}_{k}(x)=\left\|\phi_{k}\right\|_{L^{\infty}\left(B_{\xi_{k}}\left(2 \sqrt{\delta_{k}}\right)\right)}^{-1} \phi_{k}\left(\exp _{\xi_{k}}^{g_{\xi_{k}}}\left(\delta_{k} y\right)\right),
$$

and let $\tilde{x}_{k}=\frac{1}{\delta_{k}}\left(\exp _{\xi_{k}}^{g_{\xi_{k}}}\right)^{-1}\left(x_{k}\right)$. Hence, $\tilde{x}_{k} \rightarrow \tilde{x}_{0} \in \mathbb{R}^{n}$ as $k \rightarrow+\infty$. We also have $\left\|\tilde{\phi}_{k}\right\|_{L^{\infty}\left(2 \delta_{k}^{-\frac{1}{2}}\right)} \leq 1$, and using (5.4), (4.1), (5.21) and standard elliptic theory, we obtain that $\tilde{\phi}_{k}$ converges in $C_{l o c}^{1}\left(\mathbb{R}^{n}\right)$, up to a subsequence, to some function $\tilde{\phi}_{0}$ satisfying $\left|\tilde{\phi}_{0}\left(\tilde{x}_{0}\right)\right|=1$ and

$$
\triangle_{\xi} \tilde{\phi}_{0}-\left(2^{*}-1\right) f\left(\xi_{0}\right) U_{\xi_{0}}^{2^{*}-2} \tilde{\phi}_{0}=\sum_{i=0}^{n} \tilde{\lambda}_{i} \triangle_{\xi} V_{i, \xi_{0}}
$$

where $\xi_{0}=\lim _{k \rightarrow+\infty} \xi_{k}, U_{\xi_{0}}$ is defined in (2.20), $V_{i, \xi_{0}}$ is as in (2.18) and where we have let, up to a subsequence:

$$
\tilde{\lambda}_{i}=\lim _{k \rightarrow+\infty} \frac{\lambda_{k}^{i}}{\delta_{k}^{\frac{n-2}{2}}\left\|\phi_{k}\right\|_{L^{\infty}\left(B_{\xi_{k}}\left(2 \sqrt{\delta_{k}}\right)\right)}} .
$$

Note that this limit exists, up to a subsequence, by (5.17) and (5.21). Also, in (5.24), $\xi$ stands for the Euclidean metric in $\mathbb{R}^{n}$. Passing (5.23) to the limit also gives that:

$$
\left|\tilde{\phi}_{0}(y)\right| \lesssim(1+|y|)^{2-n} \text { for any } y \in \mathbb{R}^{n} \text {. }
$$


With the latter estimate we can integrate (5.24) against $V_{i, \xi_{0}}$ for any $0 \leq i \leq n$. Since $V_{i, \xi_{0}}$ solves (2.19) there holds $\tilde{\lambda}_{i}=0$ and thus $\tilde{\phi}_{0}$ satisfies:

$$
\triangle_{\xi} \tilde{\phi}_{0}=\left(2^{*}-1\right) f\left(\xi_{0}\right) U_{\xi_{0}}^{2^{*}-2} \tilde{\phi}_{0} \cdot,
$$

Again with (5.25) this shows that $\tilde{\phi}_{0} \in H^{1}(M)$ and then the Bianchi-Egnell [5] classification result applies and gives that

$$
\tilde{\phi}_{0} \in \operatorname{Span}\left\{V_{i, \xi_{0}}, 0 \leq i \leq n\right\} .
$$

To conclude the proof of Claim $\left[5.2\right.$ we now show that $\tilde{\phi}_{0} \in \operatorname{Span}\left\{V_{i, \xi}, 0 \leq i \leq n\right\}^{\perp}$. With (5.27) this will show that $\tilde{\phi}_{0} \equiv 0$, thus contradicting the fact that $\left|\tilde{\phi}_{0}\left(\tilde{x}_{0}\right)\right|=1$. By (3.8), $\phi_{k} \in K_{k}^{\perp}$, where $K_{k}=K_{k, t_{k}, \xi_{k}}$ is defined in (2.22). Hence for any $0 \leq i \leq n$ there holds:

$$
\int_{M}\left(\left\langle\nabla Z_{i, k}, \nabla \phi_{k}\right\rangle_{g}+h Z_{i, k} \phi_{k}\right) d v_{g}=0 .
$$

Let $R>0$ and $0 \leq i \leq n$. Integrating by parts the latter equation gives

$$
\begin{aligned}
& \int_{B_{\xi_{k}}\left(R \delta_{k}\right)}\left\langle\nabla Z_{i, k}, \nabla \phi_{k}\right\rangle_{g}+h Z_{i, k} \phi_{k} d v_{g}=-\int_{\partial B_{\xi_{k}}\left(R \delta_{k}\right)} \phi_{k} \partial_{\nu} Z_{i, k} d \sigma_{g} \\
& -\int_{M \backslash B_{\xi_{k}}\left(R \delta_{k}\right)}\left(h-c_{n} S_{g}\right) Z_{i, k} \phi_{k} d v_{g}-\int_{M \backslash B_{\xi_{k}}\left(R \delta_{k}\right)}\left(\triangle_{g}+c_{n} S_{g}\right) Z_{i, k} \phi_{k} d v_{g} .
\end{aligned}
$$

Using (3.9) and Hölder's inequality we get, with (5.21), that:

$$
\begin{aligned}
\left|\int_{M \backslash B_{\xi_{k}}\left(R \delta_{k}\right)}\left(h-c_{n} S_{g}\right) Z_{i, k} \phi_{k} d v_{g}\right| & \lesssim\left\|h-c_{n} S_{g}\right\|_{L^{\infty}\left(2 r_{k}\right)} \eta \delta_{k}^{2} \varepsilon_{k} \\
& =o\left(\delta_{k}^{\frac{n-2}{2}}\left\|\phi_{k}\right\|_{L^{\infty}\left(B_{\xi_{k}}\left(2 \sqrt{\delta_{k}}\right)\right)}\right) .
\end{aligned}
$$

Using (15.23) we have:

$$
\left|\int_{\partial B_{\xi_{k}}\left(R \delta_{k}\right)} \phi_{k} \partial_{\nu} Z_{i, k} d \sigma_{g}\right| \lesssim\left(\frac{1}{(1+R)^{n-2}}+o(1)\right) \delta_{k}^{\frac{n-2}{2}}\left\|\phi_{k}\right\|_{L^{\infty}\left(B_{\xi_{k}}\left(2 \sqrt{\delta_{k}}\right)\right)} .
$$

We now compute the third integral in (5.28) by using (9.2) below. By Proposition 4.1 and (2.15) we have

$$
\begin{array}{r}
\int_{M \backslash B_{\xi_{k}}\left(R \delta_{k}\right)} \delta_{k}^{\frac{n}{2}} r_{k}^{-n-1} \mathbb{1}_{r_{k} \leq d_{k} \leq 2 r_{k}}\left|\phi_{k}\right| d v_{g}+\int_{M \backslash B_{\xi_{k}}\left(R \delta_{k}\right)} \delta_{k}^{\frac{n-2}{2}} r_{k}^{-n} \mathbb{1}_{r_{k} \leq d_{k} \leq 2 r_{k}}\left|\phi_{k}\right| d v_{g} \\
+\int_{M \backslash B_{\xi_{k}}\left(R \delta_{k}\right)} \delta_{k}^{\frac{n-2}{2}} \phi_{k} d v_{g}=o\left(\delta_{k}^{\frac{n-2}{2}}\right)=o\left(\delta_{k}^{\frac{n-2}{2}}\left\|\phi_{k}\right\|_{L^{\infty}\left(B_{\xi_{k}}\left(2 \sqrt{\delta_{k}}\right)\right)}\right),
\end{array}
$$

where the last equality is due to (5.21). By (2.4) there holds

$$
\left|S_{g_{\xi_{k}}}\right|(x) \lesssim d_{k}(x)^{2} \text { in } B_{\xi_{k}}\left(2 r_{k}\right)
$$

where $d_{k}$ is the Riemannian distance defined in (5.5), so that by (5.21) and (5.23) we have:

$$
\begin{aligned}
\int_{M \backslash B_{\xi_{k}}\left(R \delta_{k}\right)} c_{n} S_{g_{\xi_{k}}} \Lambda_{\xi_{k}}^{2^{*}-2} Z_{i, k} \phi_{k} d v_{g} & =\int_{B_{\xi_{k}}\left(2 r_{k}\right) \backslash B_{\xi_{k}}\left(\sqrt{\delta_{k}}\right)} c_{n} S_{g_{\xi_{k}}} \Lambda_{\xi_{k}}^{2^{*}-2} Z_{i, k} \phi_{k} d v_{g} \\
& +\int_{B_{\xi_{k}}\left(\sqrt{\delta_{k}}\right) \backslash B_{\xi_{k}}\left(R \delta_{k}\right)} c_{n} S_{g_{\xi_{k}}} \Lambda_{\xi_{k}}^{2^{*}-2} Z_{i, k} \phi_{k} d v_{g} \\
& =o\left(\delta_{k}^{\frac{n-2}{2}}\left\|\phi_{k}\right\|_{L^{\infty}\left(B_{\xi_{k}}\left(2 \sqrt{\delta_{k}}\right)\right)}\right)
\end{aligned}
$$


where the last equality is again given by (5.21). In case where $(M, g)$ is not locally conformally flat we have, using (5.21), that:

$$
\begin{aligned}
\left|\int_{M \backslash B_{\xi_{k}}\left(\sqrt{\delta_{k}}\right)} \delta_{k}^{\frac{n}{2}} \theta_{k}(\cdot)^{2-n} \mathbb{1}_{d_{k} \leq 2 r_{k}}\right| \phi_{k}\left|d v_{g}\right| & \lesssim \delta_{k}^{\frac{n}{2}}\left\|\phi_{k}\right\|_{L^{\infty}\left(\Omega_{k}\right)} \\
& =o\left(\delta_{k}^{\frac{n-2}{2}}\left\|\phi_{k}\right\|_{L^{\infty}\left(B_{\xi_{k}}\left(2 \sqrt{\delta_{k}}\right)\right)}\right),
\end{aligned}
$$

and using (5.23) we also obtain that:

$$
\left|\int_{B_{\xi_{k}}\left(\sqrt{\delta_{k}}\right) \backslash B_{\xi_{k}}\left(R \delta_{k}\right)} \delta_{k}^{\frac{n}{2}} \theta_{k}(\cdot)^{2-n} \mathbb{1}_{d_{k} \leq 2 r_{k}}\right| \phi_{k}\left|d v_{g}\right|=o\left(\delta_{k}^{\frac{n-2}{2}}\left\|\phi_{k}\right\|_{L^{\infty}\left(B_{\xi_{k}}\left(2 \sqrt{\delta_{k}}\right)\right)}\right) .
$$

Finally, by (5.21) we can write that:

$$
\begin{aligned}
\left|\int_{M \backslash B_{\xi_{k}}\left(\sqrt{\delta_{k}}\right)} f\left(\xi_{k}\right) W_{k}^{2^{*}-2} Z_{i, k} \phi_{k} d v_{g}\right| & \lesssim \delta_{k}^{\frac{n}{2}}\left\|\phi_{k}\right\|_{L^{\infty}\left(\Omega_{k}\right)}, \\
& =o\left(\delta_{k}^{\frac{n-2}{2}}\left\|\phi_{k}\right\|_{L^{\infty}\left(B_{\xi_{k}}\left(2 \sqrt{\delta_{k}}\right)\right)}\right)
\end{aligned}
$$

where $\Omega_{k}$ is as in (5.3), and using (5.23) one gets that

$$
\begin{aligned}
& \left|\int_{B_{\xi_{k}}\left(\sqrt{\delta_{k}}\right) \backslash B_{\xi_{k}}\left(R \delta_{k}\right)} f\left(\xi_{k}\right) W_{k}^{2^{*}-2} Z_{i, k} \phi_{k} d v_{g}\right| \\
& \quad \lesssim\left(\frac{1}{(1+R)^{n-2}}+o(1)\right) \delta_{k}^{\frac{n-2}{2}}\left\|\phi_{k}\right\|_{L^{\infty}\left(B_{\xi_{k}}\left(2 \sqrt{\delta_{k}}\right)\right)} .
\end{aligned}
$$

Combining (5.31), (5.32), (5.33), (5.34), (5.35) and (5.36) in (9.2) we obtain that:

$$
\left|\int_{M \backslash B_{\xi_{k}}\left(R \delta_{k}\right)}\left(\triangle_{g}+c_{n} S_{g}\right) Z_{i, k} \phi_{k} d v_{g}\right| \lesssim\left(\frac{1}{(1+R)^{n-2}}+o(1)\right) \delta_{k}^{\frac{n-2}{2}}\left\|\phi_{k}\right\|_{L^{\infty}\left(B_{\xi_{k}}\left(2 \sqrt{\delta_{k}}\right)\right)} .
$$

We now divide (5.28) by $\delta_{k}^{\frac{n-2}{2}}\left\|\phi_{k}\right\|_{L^{\infty}\left(B_{\xi_{k}}\left(2 \sqrt{\delta_{k}}\right)\right)}$. We first use (5.29), (5.30), (5.37), the $C^{1}$ convergence of $\tilde{\phi}_{k}$ towards $\tilde{\phi}_{0}$ and the expression of $Z_{i, k}$ in (2.21) to pass to the limit as $k \rightarrow+\infty$. We then use (5.25) to pass to the limit as $R \rightarrow+\infty$, to obtain that:

$$
\int_{\mathbb{R}^{n}}\left\langle\nabla \tilde{\phi}_{0}, \nabla V_{i, \xi_{0}}\right\rangle_{\text {eucl }} d x=0 \text { for all } 0 \leq i \leq n,
$$

where $\xi_{0}=\lim _{k \rightarrow+\infty} \xi_{k}$. We thus have in the end that:

$$
\tilde{\phi}_{0} \in \operatorname{Span}\left\{V_{i, \xi_{0}}, 0 \leq i \leq n\right\}^{\perp} .
$$

With (5.27), this implies that $\tilde{\phi}_{0} \equiv 0$ which contradicts the fact that $\left|\tilde{\phi}_{0}\left(\tilde{x}_{0}\right)\right|=1$, and therefore concludes the proof of Claim 5.2 .

Hence, equation (5.19) holds true. Assume first that there holds $M_{k} \lesssim 1$, where $M_{k}$ is defined in (5.20). Then (5.18) shows that, for any sequence $x_{k} \in B_{\xi_{k}}\left(2 \sqrt{\delta_{k}}\right)$, there holds:

$$
\begin{aligned}
\left|\phi_{k}\left(x_{k}\right)\right| & \lesssim\left\|\phi_{k}\right\|_{L^{\infty}\left(\Omega_{k}\right)}+\sqrt{\delta_{k}}\left\|\nabla \phi_{k}\right\|_{L^{\infty}\left(\Omega_{k}\right)}+\delta_{k}+\left(\frac{\delta_{k}}{\theta_{k}\left(x_{k}\right)}\right)^{2} \\
& +\left[\delta_{k}\|\nabla f\|_{L^{\infty}\left(2 r_{k}\right)}+\left\|h-c_{n} S_{g}\right\|_{L^{\infty}\left(2 r_{k}\right)} \theta_{k}\left(x_{k}\right)^{2}+\theta_{k}\left(x_{k}\right)^{4} \mathbb{1}_{n l c f}\right] W_{k}\left(x_{k}\right) .
\end{aligned}
$$


Assume now that $M_{k}>>1$. Then, plugging (5.20) in (5.18) yields:

$$
\begin{aligned}
\left|\phi_{k}\left(x_{k}\right)\right| & \lesssim\left\|\phi_{k}\right\|_{L^{\infty}\left(\Omega_{k}\right)}+\sqrt{\delta_{k}}\left\|\nabla \phi_{k}\right\|_{L^{\infty}\left(\Omega_{k}\right)}+\delta_{k}+\left(\frac{\delta_{k}}{\theta_{k}\left(x_{k}\right)}\right)^{2} \\
+ & {\left[\delta_{k}\|\nabla f\|_{L^{\infty}\left(2 r_{k}\right)}+\left\|h-c_{n} S_{g}\right\|_{L^{\infty}\left(2 r_{k}\right)} \theta_{k}\left(x_{k}\right)^{2}+\theta_{k}\left(x_{k}\right)^{4} \mathbb{1}_{n l c f}\right] W_{k}\left(x_{k}\right) } \\
+ & {\left[\delta_{k}^{3}+\delta_{k}^{4-\frac{n}{2}}\|\nabla f\|_{L^{\infty}\left(2 r_{k}\right)}+\delta_{k}^{5-\frac{n}{2}}\left\|h-c_{n} S_{g}\right\|_{L^{\infty}\left(2 r_{k}\right)}\right.} \\
& \left.+\delta_{k}^{7-\frac{n}{2}} \mathbb{1}_{n l c f}\right] \theta_{k}\left(x_{k}\right)^{-2}
\end{aligned}
$$

We proceed again as we did to obtain (5.23): we use (5.39) to obtain a better estimate of $I_{1}$ in (5.8), which in turn gives with (5.7) an improved pointwise estimate of $\left|\phi_{k}\right|$. After a finite number of iterations one obtains:

$$
\begin{aligned}
& \left|\phi_{k}\left(x_{k}\right)\right| \lesssim\left\|\phi_{k}\right\|_{L^{\infty}\left(\Omega_{k}\right)}+\sqrt{\delta_{k}}\left\|\nabla \phi_{k}\right\|_{L^{\infty}\left(\Omega_{k}\right)}+\delta_{k} \\
& +\left[\delta_{k}^{\frac{n}{2}}+\delta_{k}\|\nabla f\|_{L^{\infty}\left(2 r_{k}\right)}+\left\|h-c_{n} S_{g}\right\|_{L^{\infty}\left(2 r_{k}\right)} \delta_{k}^{2}\left|\ln \left(\frac{\theta_{k}\left(x_{k}\right)}{\delta_{k}}\right)\right|\right. \\
& \left.+\left\|h-c_{n} S_{g}\right\|_{L^{\infty}\left(2 r_{k}\right)} \theta_{k}\left(x_{k}\right)^{2}+\theta_{k}\left(x_{k}\right)^{4} \mathbb{1}_{n l c f}\right] W_{k}\left(x_{k}\right)+\left(\frac{\delta_{k}}{\theta_{k}\left(x_{k}\right)}\right)^{2} .
\end{aligned}
$$

We now assume that $n=6$. The proof closely follows the $n \geq 7$ case so we only highlight the main differences. From (4.3), using (2.12), Proposition 4.1 and (9.1), it is easily seen that $\phi_{k}$ satisfies:

$$
\begin{aligned}
\left(\triangle_{g}+h\right)\left(\phi_{k}-\sum_{i=0}^{6} \lambda_{k}^{i} Z_{i, k}\right) & =(2+o(1)) f\left(u+W_{k}\right) \phi_{k} \\
& +\left(f-f\left(\xi_{k}\right)\right) W_{k}^{2}-\left(h-\frac{1}{5} S_{g}-2 f u\right) W_{k} \\
& -\frac{1}{5} S_{g_{\xi_{k}}} \Lambda_{g_{\xi_{k}}} W_{k}+O\left(\delta_{k}^{2} \mathbb{1}_{d_{k} \leq 2 r_{k}}\right)+O\left(\delta_{k}^{2} r_{k}^{-6} \mathbb{1}_{r_{k} \leq d_{k} \leq 2 r_{k}}\right) \\
& +\left(\left|\mathcal{L}_{g} T+\sigma\right|_{g}^{2}+\pi^{2}\right)\left(u_{k}^{-4}-u^{-4}\right) \\
& +\frac{\left|\mathcal{L}_{g} T_{k}+\sigma\right|_{g}^{2}-\left|\mathcal{L}_{g} T+\sigma\right|_{g}^{2}}{\left(u+W_{k}+v_{k}\right)^{4}}
\end{aligned}
$$

Let $\left(x_{k}\right)_{k}$ be a sequence of points in $B_{\xi_{k}}\left(2 \sqrt{\delta_{k}}\right)$. If $x_{k} \in \Omega_{k}$ we have as before:

$$
\left|\phi_{k}\left(x_{k}\right)\right| \leq\left\|\phi_{k}\right\|_{L^{\infty}\left(\Omega_{k}\right)} .
$$

Assume now that $x_{k} \in B_{\xi_{k}}\left(\sqrt{\delta_{k}}\right)$. Mimicking (5.10), there holds

$$
\int_{B_{\xi_{k}}\left(2 \sqrt{\delta_{k}}\right)}\left(h-\frac{1}{5} S_{g}-2 f u\right) W_{k}(y) G\left(x_{k}, y\right) d v_{g}(y) \lesssim\left\|h-\frac{1}{5} S_{g}-2 f u\right\|_{L^{\infty}\left(2 r_{k}\right)}\left(\frac{\delta_{k}}{\theta_{k}\left(x_{k}\right)}\right)^{2},
$$


so that using (5.9) - (5.13), a representation formula for $\phi_{k}-\sum_{i=0}^{6} \lambda_{k}^{i} Z_{i, k}$ in $B_{\xi_{k}}\left(2 \sqrt{\delta_{k}}\right)$ gives, with (5.41), that:

$$
\begin{aligned}
& \left|\phi_{k}-\sum_{i=0}^{6} \lambda_{k}^{i} Z_{i, k}\right|\left(x_{k}\right) \lesssim\left\|\phi_{k}\right\|_{L^{\infty}\left(\Omega_{k}\right)}+\sqrt{\delta_{k}}\left\|\nabla \phi_{k}\right\|_{L^{\infty}\left(\Omega_{k}\right)}+\sum_{i=0}^{6}\left|\lambda_{k}^{i}\right|+\delta_{k} \\
& +\delta_{k}\|\nabla f\|_{L^{\infty}\left(2 r_{k}\right)} W_{k}\left(x_{k}\right)+\left(\left\|h-\frac{1}{5} S_{g}-2 f u\right\|_{L^{\infty}\left(2 r_{k}\right)}+\left\|\phi_{k}\right\|_{L^{\infty}\left(B_{\xi_{k}}\left(2 \sqrt{\delta_{k}}\right)\right)}\right)\left(\frac{\delta_{k}}{\theta_{k}\left(x_{k}\right)}\right)^{2} .
\end{aligned}
$$

As before, the $\left|\lambda_{k}^{i}\right|$ are estimated by

$$
\begin{aligned}
\sum_{i=0}^{6}\left|\lambda_{k}^{i}\right| & \lesssim \delta_{k}^{2}\left(\left\|\phi_{k}\right\|_{L^{\infty}\left(\Omega_{k}\right)}+\sqrt{\delta_{k}}\left\|\nabla \phi_{k}\right\|_{L^{\infty}\left(\Omega_{k}\right)}\right)+\delta_{k}^{3} \\
& +\delta_{k}\|\nabla f\|_{L^{\infty}\left(2 r_{k}\right)}+\left\|h-\frac{1}{5} S_{g}-2 f u\right\|_{L^{\infty}\left(2 r_{k}\right)} \delta_{k}^{2}+\delta_{k}^{2}\left\|\phi_{k}\right\|_{L^{\infty}\left(B_{\xi_{k}}\left(2 \sqrt{\delta_{k}}\right)\right)}
\end{aligned}
$$

so that plugging (5.44) in (5.43) and using (5.42) gives, for any sequence of points $x_{k} \in B_{\xi_{k}}\left(2 \sqrt{\delta_{k}}\right)$ :

$$
\begin{aligned}
\left|\phi_{k}\left(x_{k}\right)\right| & \lesssim\left\|\phi_{k}\right\|_{L^{\infty}\left(\Omega_{k}\right)}+\sqrt{\delta_{k}}\left\|\nabla \phi_{k}\right\|_{L^{\infty}\left(\Omega_{k}\right)}+\delta_{k}\|\nabla f\|_{L^{\infty}\left(2 r_{k}\right)} W_{k}\left(x_{k}\right)+\delta_{k} \\
& +\left(\left\|h-\frac{1}{5} S_{g}-2 f u\right\|_{L^{\infty}\left(2 r_{k}\right)}+\left\|\phi_{k}\right\|_{L^{\infty}\left(B_{\xi_{k}}\left(2 \sqrt{\delta_{k}}\right)\right)}\right)\left(\frac{\delta_{k}}{\theta_{k}\left(x_{k}\right)}\right)^{2} .
\end{aligned}
$$

We now claim that there holds:

$$
\left\|\phi_{k}\right\|_{L^{\infty}\left(B_{\xi_{k}}\left(2 \sqrt{\delta_{k}}\right)\right)} \lesssim N_{k}
$$

where we have let:

$$
N_{k}=\left\|\phi_{k}\right\|_{L^{\infty}\left(\Omega_{k}\right)}+\sqrt{\delta_{k}}\left\|\nabla \phi_{k}\right\|_{L^{\infty}\left(\Omega_{k}\right)}+\delta_{k}+\delta_{k}^{-1}\|\nabla f\|_{L^{\infty}\left(2 r_{k}\right)}+\left\|h-\frac{1}{5} S_{g}-2 f u\right\|_{L^{\infty}\left(2 r_{k}\right)},
$$

and where $\Omega_{k}$ is as in (5.3). To prove (5.46), let $\left(x_{k}\right)_{k}$ be a sequence of points in $B_{\xi_{k}}\left(2 \sqrt{\delta_{k}}\right)$ satisfying $\left|\phi_{k}\left(x_{k}\right)\right|=\max _{B_{\xi_{k}}\left(2 \sqrt{\delta_{k}}\right)}\left|\phi_{k}\right|$. Estimate (5.46) is trivially satisfied if $x_{k} \in \Omega_{k}$, so we assume in the following that $x_{k} \in B_{\xi_{k}}\left(\sqrt{\delta_{k}}\right)$. We proceed by contradiction and assume that there holds:

$$
\left|\phi_{k}\left(x_{k}\right)\right|=\left\|\phi_{k}\right\|_{L^{\infty}\left(B_{\xi_{k}}\left(2 \sqrt{\delta_{k}}\right)\right)}>>N_{k}
$$

as $k \rightarrow+\infty$, where $N_{k}$ is defined in (5.47). Let $\left(y_{k}\right)_{k}$ be any other sequence of points in $B_{\xi_{k}}\left(\sqrt{\delta_{k}}\right)$. Proceeding as in (5.22) (5.23) we obtain that there holds:

$$
\left|\phi_{k}\left(y_{k}\right)\right| \lesssim\left(\left(\frac{\delta_{k}}{\theta_{k}\left(y_{k}\right)}\right)^{4}+o(1)\right)\left\|\phi_{k}\right\|_{L^{\infty}\left(B_{\xi_{k}}\left(2 \sqrt{\delta_{k}}\right)\right)},
$$

so that (5.49) applied to the sequence $x_{k}$ given by (5.48) yields:

$$
\theta_{k}\left(x_{k}\right) \lesssim \delta_{k}
$$

where $\theta_{k}$ is as in (4.25). Define now, for $y \in B_{0}\left(2 \delta_{k}^{-\frac{1}{2}}\right)$ :

$$
\tilde{\phi}_{k}(x)=\left\|\phi_{k}\right\|_{L^{\infty}\left(B_{\xi_{k}}\left(2 \sqrt{\delta_{k}}\right)\right)}^{-1} \phi_{k}\left(\exp _{\xi_{k}}^{g_{\xi_{k}}}\left(\delta_{k} y\right)\right),
$$

and let $\tilde{x}_{k}=\frac{1}{\delta_{k}}\left(\exp _{\xi_{k}}^{g_{\xi_{k}}}\right)^{-1}\left(x_{k}\right)$. Here again, $\tilde{x}_{k} \rightarrow \tilde{x}_{0} \in \mathbb{R}^{n}$ as $k \rightarrow+\infty$. The arguments that led to (5.27) adapt therefore with no modification and we obtain in the end that $\tilde{\phi}_{k}$ converges in $C_{l o c}^{1}\left(\mathbb{R}^{n}\right)$, up to a subsequence, to some function $\tilde{\phi}_{0}$ satisfying $\left|\tilde{\phi}_{0}\left(\tilde{x}_{0}\right)\right|=1$ and

$$
\tilde{\phi}_{0} \in \operatorname{Span}\left\{V_{i, \xi_{0}}, 0 \leq i \leq 6\right\} \text {. }
$$


To conclude the proof of (5.46) we now show that $\tilde{\phi}_{0} \in \operatorname{Span}\left\{V_{i, \xi}, 0 \leq i \leq 6\right\}^{\perp}$. By (3.8), $\phi_{k} \in K_{k}^{\perp}$, where $K_{k}=K_{k, t_{k}, \xi_{k}}$ is defined in (2.22). Hence for any $0 \leq i \leq 6$ and for any $R>0$ fixed, there holds:

$$
\begin{aligned}
& \int_{B_{\xi_{k}}\left(R \delta_{k}\right)}\left\langle\nabla Z_{i, k}, \nabla \phi_{k}\right\rangle_{g}+h Z_{i, k} \phi_{k} d v_{g}=-\int_{\partial B_{\xi_{k}}\left(R \delta_{k}\right)} \phi_{k} \partial_{\nu} Z_{i, k} d \sigma_{g} \\
& -\int_{M \backslash B_{\xi_{k}}\left(R \delta_{k}\right)}\left(h-\frac{1}{5} S_{g}\right) Z_{i, k} \phi_{k} d v_{g}-\int_{M \backslash B_{\xi_{k}}\left(R \delta_{k}\right)}\left(\triangle_{g}+\frac{1}{5} S_{g}\right) Z_{i, k} \phi_{k} d v_{g} .
\end{aligned}
$$

By (5.48) and (2.21) one has that:

$$
\int_{M \backslash B_{\xi_{k}}\left(\sqrt{\delta_{k}}\right)}\left(h-\frac{1}{5} S_{g}\right) Z_{i, k} \phi_{k} d v_{g}=o\left(\delta_{k}^{2}\left\|\phi_{k}\right\|_{L^{\infty}\left(B_{\xi_{k}}\left(2 \sqrt{\delta_{k}}\right)\right)}\right),
$$

while using (5.49) there holds that:

$$
\int_{B_{\xi_{k}}\left(\sqrt{\delta_{k}}\right) \backslash B_{\xi_{k}}\left(R \delta_{k}\right)}\left(h-c_{n} S_{g}\right) Z_{i, k} \phi_{k} d v_{g}=o\left(\delta_{k}^{2}\left\|\phi_{k}\right\|_{L^{\infty}\left(B_{\xi_{k}}\left(2 \sqrt{\delta_{k}}\right)\right)}\right),
$$

that

$$
\left|\int_{\partial B_{\xi_{k}}\left(R \delta_{k}\right)} \phi_{k} \partial_{\nu} Z_{i, k} d \sigma_{g}\right| \lesssim\left(\frac{1}{(1+R)^{4}}+o(1)\right) \delta_{k}^{2}\left\|\phi_{k}\right\|_{L^{\infty}\left(B_{\xi_{k}}\left(2 \sqrt{\delta_{k}}\right)\right)},
$$

and, with (2.15) and (5.48), that

$$
\begin{aligned}
\int_{M \backslash B_{\xi_{k}}\left(R \delta_{k}\right)} \delta_{k}^{3} r_{k}^{-7} \mathbb{1}_{r_{k} \leq} & d_{k} \leq 2 r_{k}\left|\phi_{k}\right| d v_{g}+\int_{M \backslash B_{\xi_{k}}\left(R \delta_{k}\right)} \delta_{k}^{2} r_{k}^{-6} \mathbb{1}_{r_{k} \leq d_{k} \leq 2 r_{k}}\left|\phi_{k}\right| d v_{g} \\
& +\int_{M \backslash B_{\xi_{k}}\left(R \delta_{k}\right)} \delta_{k}^{2} \phi_{k} d v_{g}=o\left(\delta_{k}^{2}\left\|\phi_{k}\right\|_{L^{\infty}\left(B_{\xi_{k}}\left(2 \sqrt{\delta_{k}}\right)\right)}\right) .
\end{aligned}
$$

Using (2.4), (5.48) and (5.49) we get also that:

$$
\begin{aligned}
\int_{M \backslash B_{\xi_{k}}\left(R \delta_{k}\right)} \frac{1}{5} & S_{g_{\xi_{k}}} \Lambda_{g_{\xi_{k}}} Z_{i, k} \phi_{k} d v_{g} \\
& =\int_{M \backslash B_{\xi_{k}}\left(\sqrt{\delta_{k}}\right)} \frac{1}{5} S_{g_{\xi_{k}}} \Lambda_{g_{\xi_{k}}} Z_{i, k} \phi_{k} d v_{g}+\int_{B_{\xi_{k}}\left(\sqrt{\delta_{k}}\right) \backslash B_{\xi_{k}}\left(R \delta_{k}\right)} \frac{1}{5} S_{g_{\xi_{k}}} \Lambda_{g_{\xi_{k}}} Z_{i, k} \phi_{k} d v_{g} \\
& =o\left(\delta_{k}^{2}\left\|\phi_{k}\right\|_{L^{\infty}\left(B_{\xi_{k}}\left(2 \sqrt{\delta_{k}}\right)\right)}\right) .
\end{aligned}
$$

Similarly, we get that

that

$$
\int_{M \backslash B_{\xi_{k}}\left(R \delta_{k}\right)} \delta_{k}^{3} \theta_{k}(\cdot)^{-4} \mathbb{1}_{d_{k} \leq 2 r_{k}}\left|\phi_{k}\right| d v_{g}=o\left(\delta_{k}^{2}\left\|\phi_{k}\right\|_{L^{\infty}\left(B_{\xi_{k}}\left(2 \sqrt{\delta_{k}}\right)\right)}\right)
$$

and, using (5.49), that

$$
\begin{aligned}
\left|\int_{M \backslash B_{\xi_{k}}\left(\sqrt{\delta_{k}}\right)} f\left(\xi_{k}\right) W_{k} Z_{i, k} \phi_{k} d v_{g}\right| & \lesssim \delta_{k}^{3}\left\|\phi_{k}\right\|_{L^{\infty}\left(\Omega_{k}\right)}, \\
& =o\left(\delta_{k}^{2}\left\|\phi_{k}\right\|_{L^{\infty}\left(B_{\xi_{k}}\left(2 \sqrt{\delta_{k}}\right)\right)}\right),
\end{aligned}
$$

$$
\left|\int_{B_{\xi_{k}}\left(\sqrt{\delta_{k}}\right) \backslash B_{\xi_{k}}\left(R \delta_{k}\right)} f\left(\xi_{k}\right) W_{k} Z_{i, k} \phi_{k} d v_{g}\right| \lesssim\left(\frac{1}{(1+R)^{4}}+o(1)\right) \delta_{k}^{2}\left\|\phi_{k}\right\|_{L^{\infty}\left(B_{\xi_{k}}\left(2 \sqrt{\delta_{k}}\right)\right)},
$$

so that passing to the limit as $k \rightarrow+\infty$ and then as $R \rightarrow+\infty$ gives, with (5.51):

$$
\tilde{\phi}_{0} \in \operatorname{Span}\left\{V_{i, \xi_{0}}, 0 \leq i \leq 6\right\}^{\perp},
$$


which is a contradiction with (5.50) since $\left|\tilde{\phi}_{0}\left(\tilde{x}_{0}\right)\right|=1$. Therefore (5.46) is proven, and an iteration argument as for the $n \geq 7$ case gives:

$$
\begin{aligned}
& \left|\phi_{k}\left(x_{k}\right)\right| \lesssim\left\|\phi_{k}\right\|_{L^{\infty}\left(\Omega_{k}\right)}+\sqrt{\delta_{k}}\left\|\nabla \phi_{k}\right\|_{L^{\infty}\left(\Omega_{k}\right)}+\delta_{k}+\left[\delta_{k}^{3}+\delta_{k}\|\nabla f\|_{L^{\infty}\left(2 r_{k}\right)}\right. \\
& \left.+\left\|h-\frac{1}{5} S_{g}-2 f u\right\|_{L^{\infty}\left(2 r_{k}\right)} \delta_{k}^{2}\left|\ln \left(\frac{\theta_{k}\left(x_{k}\right)}{\delta_{k}}\right)\right|+\left\|h-\frac{1}{5} S_{g}-2 f u\right\|_{L^{\infty}\left(2 r_{k}\right)} \theta_{k}\left(x_{k}\right)^{2}\right] W_{k}\left(x_{k}\right) .
\end{aligned}
$$

To conclude the proof of Proposition 5.1 it remains to improve (5.40) and (5.52) into the final estimates (5.1) and (5.2). We only prove the $n \geq 7$ case, as the six-dimensional case follows from similar arguments. Let $\left(x_{k}\right)_{k}$ be any sequence of points in $B_{\xi_{k}}\left(2 \sqrt{\delta_{k}}\right)$. We write a Green representation formula and differentiate it at $x_{k}$. As in (5.9) (5.13) one obtains that:

$$
\begin{aligned}
& \left|\nabla\left(\psi_{k}-\sum_{j=0}^{n} \lambda_{k}^{i} Z_{i, k}\right)\left(x_{k}\right)\right| \lesssim \frac{1}{\sqrt{\delta_{k}}}\left\|\phi_{k}\right\|_{L^{\infty}\left(\Omega_{k}\right)}+\left\|\nabla \phi_{k}\right\|_{L^{\infty}\left(\Omega_{k}\right)}+\sqrt{\delta_{k}} \\
& +\left[\delta_{k}^{\frac{n}{2}}+\delta_{k}\|\nabla f\|_{L^{\infty}\left(2 r_{k}\right)}+\left\|h-c_{n} S_{g}\right\|_{L^{\infty}\left(2 r_{k}\right)} \theta_{k}\left(x_{k}\right)^{2}+\theta_{k}\left(x_{k}\right)^{4} \mathbb{1}_{n l c f}\right] \delta_{k}^{\frac{n-2}{2}} \theta_{k}\left(x_{k}\right)^{1-n} \\
& +\int_{B_{\xi_{k}}\left(2 \sqrt{\delta_{k}}\right)} f\left|u_{k}^{2^{*}-1}-W_{k}^{2^{*}-1}-u^{2^{*}-1}\right|(y) \nabla_{x} G\left(x_{k}, y\right) d v_{g}(y) .
\end{aligned}
$$

The last term is then estimated with (5.40) to obtain (5.1).

Note that using Claim 5.2 and (5.46) in (5.17) and (5.44) yields:

- If $n \geq 7$ :

$$
\begin{aligned}
\sum_{i=0}^{n}\left|\lambda_{k}^{i}\right| & \lesssim \delta_{k}^{\frac{n-2}{2}}\left(\left\|\phi_{k}\right\|_{L^{\infty}\left(\Omega_{k}\right)}+\sqrt{\delta_{k}}\left\|\nabla \phi_{k}\right\|_{L^{\infty}\left(\Omega_{k}\right)}\right)+\delta_{k}^{\frac{n-2}{2}} \\
& +\delta_{k}\|\nabla f\|_{L^{\infty}\left(2 r_{k}\right)}+\left\|h-c_{n} S_{g}\right\|_{L^{\infty}\left(2 r_{k}\right)} \delta_{k}^{2}+\delta_{k}^{4} \mathbb{1}_{n l c f} .
\end{aligned}
$$

- If $n=6$ :

$$
\begin{aligned}
\sum_{i=0}^{6}\left|\lambda_{k}^{i}\right| & \lesssim \delta_{k}^{2}\left(\left\|\phi_{k}\right\|_{L^{\infty}\left(\Omega_{k}\right)}+\sqrt{\delta_{k}}\left\|\nabla \phi_{k}\right\|_{L^{\infty}\left(\Omega_{k}\right)}\right)+\delta_{k}^{3} \\
& +\delta_{k}\|\nabla f\|_{L^{\infty}\left(2 r_{k}\right)}+\left\|h-\frac{1}{5} S_{g}-2 f u\right\|_{L^{\infty}\left(2 r_{k}\right)} \delta_{k}^{2}
\end{aligned}
$$

As a consequence of these refined local estimates we are now in position to obtain global pointwise estimates on $\phi_{k}$ in the whole manifold $M$ :

Proposition 5.3. Let $D>0$ and $\left(\varepsilon_{k}\right)_{k} \in \mathcal{E}$ and assume that $\varepsilon_{k}>>\mu_{k}^{\frac{3}{2}}$ as $k \rightarrow+\infty$, where $\mu_{k}$ is defined in (2.5). Let $\left(t_{k}, \xi_{k}\right)_{k}$ be a sequence in $[1 / D, D] \times M$, let $v_{k} \in F_{k}=F\left(\varepsilon_{k}, t_{k}, \xi_{k}\right)$ and let $\phi_{k}=\phi_{k}\left(t_{k}, \xi_{k}, v_{k}\right)$ be given by Proposition 3.3. Let $\left(x_{k}\right)_{k}$ be any sequence of points in $M$. There holds then:

$$
\left|\phi_{k}\left(x_{k}\right)\right| \leq C\left(\delta_{k}+\eta \varepsilon_{k}\right)\left(u\left(x_{k}\right)+W_{k}\left(x_{k}\right)\right),
$$

where $\eta$ is as in (2.11), for some positive constant $C$ independent of $\eta$ and $k$. As a consequence, we have the following gradient estimate:

$$
\left|\nabla \phi_{k}\left(x_{k}\right)\right| \leq C\left(\delta_{k}+\eta \varepsilon_{k}\right)\left(1+\delta_{k}^{\frac{n-2}{2}} \theta_{k}\left(x_{k}\right)^{1-n}\right) .
$$


Note that the constant $C$ appearing in the statement of Proposition 5.3 a priori depends on $g, u_{0}$ (as in (2.8) $), h, f, \sigma, \pi$, but does not depend on the choice of the sequences $\left(\varepsilon_{k}\right)_{k},\left(t_{k}\right)_{k},\left(\xi_{k}\right)_{k}$ and $\left(v_{k}\right)_{k}$.

Proof. Let $D>0$ and $\left(\varepsilon_{k}\right)_{k} \in \mathcal{E}$ and assume that $\varepsilon_{k}>>\mu_{k}^{\frac{3}{2}}$ as $k \rightarrow+\infty$, where $\mu_{k}$ is defined in (2.5). Let $\left(t_{k}, \xi_{k}\right)_{k}$ be a sequence in $[1 / D, D] \times M$, let $v_{k} \in F_{k}=F\left(\varepsilon_{k}, t_{k}, \xi_{k}\right)$ and let $\phi_{k}=\phi_{k}\left(t_{k}, \xi_{k}, v_{k}\right)$ be given by Proposition 3.3 . Define:

$$
\nu_{k}=\left\|\frac{\phi_{k}}{u+W_{k}}\right\|_{L^{\infty}(M)} .
$$

Proposition 4.1 shows that $\nu_{k}=o(1)$ as $k \rightarrow+\infty$. We let in the following, for any $v \in H^{1}(M)$ :

$$
L_{u}(v)=\triangle_{g} v+\left[h-\left(2^{*}-1\right) f u^{2^{*}-2}+\left(2^{*}+1\right) \frac{\left|\mathcal{L}_{g} T+\sigma\right|_{g}^{2}+\pi^{2}}{u^{2^{*}+2}}\right] v .
$$

$L_{u}$ is the linearized operator of the scalar equation of (2.12) at $u$.

As before, assume first that $n \geq 7$. It is easily seen from (5.4) that $\phi_{k}$ satisfies:

$$
\begin{aligned}
L_{u} \phi_{k} & =\sum_{i=0}^{n} \lambda_{k}^{i}\left(\triangle_{g}+h\right) Z_{i, k}+f\left(u_{k}^{2^{*}-1}-W_{k}^{2^{*}-1}-\left(2^{*}-1\right) u^{2^{*}-2} \phi_{k}-u^{2^{*}-1}\right) \\
& +\left(f-f\left(\xi_{k}\right)\right) W_{k}^{2^{*}-1}-\left(h-c_{n} S_{g}\right) W_{k} \\
& -c_{n} S_{g_{\xi_{k}}} \Lambda_{g_{\xi_{k}}}^{2^{*}-2} W_{k}+O\left(\delta_{k}^{\frac{n-2}{2}} \mathbb{1}_{d k \leq 2 r_{k}}\right)+O\left(\delta_{k}^{\frac{n-2}{2}} r_{k}^{-n} \mathbb{1}_{r_{k} \leq d_{k} \leq 2 r_{k}}\right) \\
& +\left(\left|\mathcal{L}_{g} T+\sigma\right|_{g}^{2}+\pi^{2}\right)\left(u_{k}^{-2^{*}-1}-u^{-2^{*}-1}+\left(2^{*}+1\right) u^{-2^{*}-2} \phi_{k}\right) \\
& +\frac{\left|\mathcal{L}_{g} T_{k}+\sigma\right|_{g}^{2}-\left|\mathcal{L}_{g} T+\sigma\right|_{g}^{2}}{\left(u+W_{k}+v_{k}\right)^{2^{*}+1}} .
\end{aligned}
$$

Let $\left(x_{k}\right)_{k}$ be any sequence of points in $M$. Let $G_{u}$ be the Green's function of the operator $L_{u}$ defined in (5.58). By (2.13), the operator $L_{u}$ is coercive, and therefore its Green function satisfies (see for instance Robert [43]):

$$
\frac{1}{C} d_{g_{\xi_{k}}}(x, y)^{2-n} \leq G_{u}(x, y) \leq C d_{g_{\xi_{k}}}(x, y)^{2-n},
$$

for some positive constant $C$. Note that since we assumed $|\sigma|_{g}+\pi>0$ somewhere in $M$, the constant $C$ in (5.60) does not depend on $\eta$ in (2.11), provided $\eta$ is small enough. First, by (5.54), by Proposition 4.1 and since $\left|Z_{i, k}\right| \lesssim W_{k}$ for all $0 \leq i \leq n$, we have:

$$
\begin{aligned}
\int_{M} G_{u}\left(x_{k}, y\right) & \sum_{i=0}^{n} \lambda_{k}^{i}\left(\triangle_{g}+h\right) Z_{i, k}(y) d v_{g}(y) \\
& \lesssim\left[\delta_{k}^{\frac{n-2}{2}}+\delta_{k}\|\nabla f\|_{L^{\infty}\left(2 r_{k}\right)}+\left\|h-c_{n} S_{g}\right\|_{L^{\infty}\left(2 r_{k}\right)} \delta_{k}^{2}+\delta_{k}^{4} \mathbb{1}_{n l c f}\right] \delta_{k}^{\frac{n-2}{2}} \theta_{k}\left(x_{k}\right)^{2-n} .
\end{aligned}
$$

Independently, straightforward computations using (5.60) show that there holds:

$$
\begin{aligned}
& \int_{M} G_{u}\left(x_{k}, y\right)\left[\left(f(y)-f\left(\xi_{k}\right)\right) W_{k}^{2^{*}-1}(y)+\left(h-c_{n} S_{g}\right) W_{k}(y)+c_{n} S_{g_{\xi_{k}}}(y) W_{k}(y)\right] d v_{g}(y) \\
& \quad \lesssim\left(\delta_{k}\|\nabla f\|_{L^{\infty}\left(2 r_{k}\right)}+\left\|h-c_{n} S_{g}\right\|_{L^{\infty}\left(2 r_{k}\right)} \theta_{k}\left(x_{k}\right)^{2}\right) \delta_{k}^{\frac{n-2}{2}} \theta_{k}\left(x_{k}\right)^{2-n}+\delta_{k}^{\frac{n-2}{2}} \theta_{k}\left(x_{k}\right)^{6-n} \mathbb{1}_{n l c f} .
\end{aligned}
$$

Similarly, there holds:

$$
\int_{M} G_{u}\left(x_{k}, y\right)\left[\delta_{k}^{\frac{n-2}{2}}+\delta_{k}^{\frac{n-2}{2}} r_{k}^{-n} \mathbb{1}_{r_{k} \leq d_{k} \leq 2 r_{k}}\right] d v_{g}(y)=O\left(\delta_{k}^{\frac{n-2}{2}} r_{k}^{2-n}\right),
$$


where $d_{k}$ is as in (5.5). Now, with (4.2), straightforward computations using Proposition 4.1 yield that:

$$
\begin{aligned}
& \int_{B_{\xi_{k}}\left(\sqrt{\delta_{k}}\right)} G_{u}\left(x_{k}, y\right)\left(\left|\mathcal{L}_{g} T+\sigma\right|_{g}^{2}+\pi^{2}\right) \\
& \times\left(u_{k}^{-2^{*}-1}-u^{-2^{*}-1}+\left(2^{*}+1\right) u^{-2^{*}-2} \phi_{k}\right)(y) d v_{g}(y) \lesssim \delta_{k}^{\frac{n}{2}} \theta_{k}\left(x_{k}\right)^{2-n}+\delta_{k}^{\frac{n-2}{2}} \theta_{k}\left(x_{k}\right)^{4-n},
\end{aligned}
$$

while using (4.2), Proposition 4.1 and the definition of $\nu_{k}$ in (5.57) there holds:

$$
\begin{aligned}
\int_{M \backslash B_{\xi_{k}}\left(\sqrt{\delta_{k}}\right)} & G_{u}\left(x_{k}, y\right)\left(\left|\mathcal{L}_{g} T+\sigma\right|_{g}^{2}+\pi^{2}\right) \\
& \times\left(u_{k}^{-2^{*}-1}-u^{-2^{*}-1}+\left(2^{*}+1\right) u^{-2^{*}-2} \phi_{k}\right)(y) d v_{g}(y) \\
& \lesssim \delta_{k}^{\frac{n-2}{2}} \theta_{k}\left(x_{k}\right)^{4-n}+\nu_{k}^{2} .
\end{aligned}
$$

Similarly, using (4.2) and the definition of $\nu_{k}$ in (5.57) one obtains that:

$$
\begin{gathered}
\int_{M \backslash B_{\xi_{k}}\left(\sqrt{\delta_{k}}\right)} G_{u}\left(x_{k}, y\right) f\left(u_{k}^{2^{*}-1}-W_{k}^{2^{*}-1}-\left(2^{*}-1\right) u^{2^{*}-2} \phi_{k}-u^{2^{*}-1}\right)(y) d v_{g}(y) \\
\lesssim \delta_{k}^{\frac{n-2}{2}} \theta_{k}\left(x_{k}\right)^{4-n}+\nu_{k}^{2} .
\end{gathered}
$$

Using Proposition 5.1 and estimating $\left\|\phi_{k}\right\|_{L^{\infty}\left(\Omega_{k}\right)}+\sqrt{\delta_{k}}\left\|\nabla \phi_{k}\right\|_{L^{\infty}\left(\Omega_{k}\right)}$ with Proposition 4.1 gives that:

$$
\begin{aligned}
\int_{B_{\xi_{k}}\left(\sqrt{\delta_{k}}\right)} & G_{u}\left(x_{k}, y\right) f\left(u_{k}^{2^{*}-1}-W_{k}^{2^{*}-1}-\left(2^{*}-1\right) u^{2^{*}-2} \phi_{k}-u^{2^{*}-1}\right)(y) d v_{g}(y) \\
& \lesssim\left(\frac{\delta_{k}}{\theta_{k}\left(x_{k}\right)}\right)^{2}+\left[\delta_{k}^{\frac{n}{2}}+\delta_{k}\|\nabla f\|_{L^{\infty}\left(2 r_{k}\right)}+\left\|h-c_{n} S_{g}\right\|_{L^{\infty}\left(2 r_{k}\right)} \delta_{k}^{2}\left|\ln \left(\delta_{k}\right)\right|\right. \\
& \left.+\delta_{k}^{2} \theta_{k}\left(x_{k}\right) \mathbb{1}_{n l c f}\right] \delta_{k}^{\frac{n-2}{2}} \theta_{k}\left(x_{k}\right)^{2-n} .
\end{aligned}
$$

Finally, using again (9.11) below and since $v_{k} \in F_{k}$, there holds that:

$$
\int_{B_{\xi_{k}}\left(\sqrt{\delta_{k}}\right)} G_{u}\left(x_{k}, y\right) \frac{\left|\mathcal{L}_{g} T_{k}+\sigma\right|_{g}^{2}-\left|\mathcal{L}_{g} T+\sigma\right|_{g}^{2}}{\left(u+W_{k}+v_{k}\right)^{2+}}(y) d v_{g}(y) \lesssim \delta_{k}^{\frac{n}{2}} \theta_{k}\left(x_{k}\right)^{2-n}+\delta_{k} \varepsilon_{k}
$$

and that

$$
\int_{M \backslash B_{\xi_{k}}\left(\sqrt{\delta_{k}}\right)} G_{u}\left(x_{k}, y\right) \frac{\left|\mathcal{L}_{g} T_{k}+\sigma\right|_{g}^{2}-\left|\mathcal{L}_{g} T+\sigma\right|_{g}^{2}}{\left(u+W_{k}+v_{k}\right)^{2^{*}+1}}(y) d v_{g}(y) \lesssim \delta_{k}^{\frac{n}{2}} \theta_{k}\left(x_{k}\right)^{2-n}+\delta_{k}^{\frac{n-2}{2}} \theta_{k}\left(x_{k}\right)^{4-n}+\eta \varepsilon_{k},
$$

where $\eta$ is as in (2.11). Writing a Green's representation formula for (5.59) together with (5.61) (5.69), with Proposition 4.1 and with (2.15) gives then:

$$
\left|\phi_{k}\left(x_{k}\right)\right| \lesssim \eta \varepsilon_{k}+\nu_{k}^{2}+\delta_{k}\left(u\left(x_{k}\right)+W_{k}\left(x_{k}\right)\right)
$$

where $\nu_{k}$ is as in (5.57). Also, to obtain (5.70) we used that there holds, for any $x \in M$ :

$$
\left(\frac{\delta_{k}}{\theta_{k}\left(x_{k}\right)}\right)^{2}+\delta_{k}^{\frac{n-2}{2}} \theta_{k}(x)^{4-n}+\delta_{k}^{\frac{n}{2}} \theta_{k}(x)^{2-n} \leq C \delta_{k}\left(u(x)+W_{k}(x)\right),
$$


for some positive constant $C$ independent of $\eta$ and $k$. Coming back to the definition of $\nu_{k}$ in (5.57), it remains to apply (5.70) at the sequence $\left(x_{k}\right)_{k}$ of points of $M$ where $\nu_{k}$ is attained. Since, by Proposition 4.1 there holds that $\nu_{k} \rightarrow 0$ as $k \rightarrow+\infty$, we obtain in the end that

$$
\nu_{k} \lesssim \delta_{k}+\eta \varepsilon_{k},
$$

which concludes the proof of (5.56) when $n \geq 7$.

Assume now that $n=6$. Rewrite (5.4) as:

$$
\begin{aligned}
L_{u} \phi_{k} & =\sum_{i=0}^{6} \lambda_{k}^{i}\left(\triangle_{g}+h\right) Z_{i, k}+2 f W_{k} \phi_{k}+f \phi_{k}^{2}+\left(f-f\left(\xi_{k}\right)\right) W_{k}^{2} \\
& -\left(h-\frac{1}{5} S_{g}-2 f u\right) W_{k}-\frac{1}{5} S_{g_{\xi_{k}}} \Lambda_{\xi_{k}} W_{k}+O\left(\delta_{k}^{2} \mathbb{1}_{d k \leq 2 r_{k}}\right)+O\left(\delta_{k}^{2} r_{k}^{-6} \mathbb{1}_{r_{k} \leq d_{k} \leq 2 r_{k}}\right) \\
& +\left(\left|\mathcal{L}_{g} T+\sigma\right|_{g}^{2}+\pi^{2}\right)\left(u_{k}^{-4}-u^{-4}+4 u^{-5} \phi_{k}\right) \\
& +\frac{\left|\mathcal{L}_{g} T_{k}+\sigma\right|_{g}^{2}-\left|\mathcal{L}_{g} T+\sigma\right|_{g}^{2}}{\left(u+W_{k}+v_{k}\right)^{4}} .
\end{aligned}
$$

Let $\left(x_{k}\right)_{k}$ be any sequence of points in $M$. As before, a Green's representation formula for (5.72) using Propositions 4.1 and 5.1 and (2.15) gives now the following estimate:

$$
\begin{aligned}
\left|\phi_{k}\left(x_{k}\right)\right| & \lesssim\left(\frac{\delta_{k}}{\theta_{k}\left(x_{k}\right)}\right)^{2}+\delta_{k}+\varepsilon_{k}+\nu_{k}^{2}+\left(\delta_{k}+\delta_{k}^{2}\left|\ln \delta_{k}\right|\left\|h-\frac{1}{5} S_{g}-2 f u\right\|_{L^{\infty}\left(2 r_{k}\right)}+\nu_{k}^{2}\right) \delta_{k}^{2} \theta_{k}\left(x_{k}\right)^{-4} \\
& \lesssim\left(\delta_{k}+\eta \varepsilon_{k}+\nu_{k}^{2}\right)\left(u+W_{k}\right)\left(x_{k}\right) .
\end{aligned}
$$

Applying (5.73) at the sequence of points where $\nu_{k}$ as in (5.57) is attained concludes the proof of (5.56) for the 6-dimensional case.

The gradient estimates in Proposition 5.3 are obtained from (5.56) by a representation formula argument as before.

Proposition 5.1 provides more information than needed to just prove Proposition 5.3. The precision of estimates (5.1) and (5.2) will turn out to be crucial in section 7 to obtain precise asymptotic expansions of the $\lambda_{k}^{i}$.

It is worth noting that Propositions 5.1 and 5.3 do depend on the choice of $X$ given by (2.24) (mostly to obtain pointwise estimates on the source term) but do not use the specific form of the other coefficients, e.g. of $h$ and $f$ defined in (2.7), (2.10) and (2.23). In particular, Propositions 5.1 and 5.3 still hold true for arbitrary choices of $h, \pi \in C^{0}(M), f \in C^{1}(M), \sigma \in C^{0}(M)$ and $Y \in C^{0}(M)$.

\section{Global fixed-point argument and Resolution of the Reduced problem}

Proposition 5.3 shows that we have pointwise estimates on the remainder $\phi_{k}$ defined by Proposition 3.3 which only depend on the data $\mu_{k}$ and $\varepsilon_{k}$, and not on the chosen sequences $\left(t_{k}, \xi_{k}\right)_{k}$ or $\left(v_{k}\right)_{k}$.

In this section we crucially use this result to show that Banach-Picard's fixed-point theorem applies to the mapping $v_{k} \mapsto \phi_{k}$ and yields a solution to the reduced problem for system (1.1). The main result of this section is the following:

Proposition 6.1. Let $D>0$. Assume that $\eta$ and $\alpha$ defined in (2.11) and (2.25) are small enough. There exists $k_{0} \in \mathbb{N}$ such that for any sequence $\left(t_{k}, \xi_{k}\right)_{k} \in[1 / D, D] \times M$ and for any $k \geq k_{0}$, there 
exists a function $\phi_{k}=\phi_{k}\left(t_{k}, \xi_{k}\right) \in K_{k, t_{k}, \xi_{k}}^{\perp}$ that satisfies the following system of equations:

$$
\left\{\begin{array}{l}
\Pi_{K_{k, t_{k}, \xi_{k}}^{\perp}}\left[u_{k}-\left(\triangle_{g}+h\right)^{-1}\left(f u_{k}^{2^{*}-1}+\frac{\left|\mathcal{L}_{g} T_{k}+\sigma\right|_{g}^{2}+\pi^{2}}{u_{k}^{2^{*}+1}}\right)\right]=0, \\
\vec{\triangle}_{g} T_{k}=u_{k}^{2^{*}} X+Y
\end{array}\right.
$$

where, as in (4.2), we have let $u_{k}=u+W_{k, t_{k}, \xi_{k}}+\phi_{k}\left(t_{k}, \xi_{k}\right)$. In addition, there exists a positive constant $C$, independent of $\left(t_{k}, \xi_{k}\right)_{k}$ such that there holds:

$$
\left\|\phi_{k}\left(t_{k}, \xi_{k}\right)\right\|_{H^{1}(M)} \leq C \delta_{k} \text { and }\left|\phi_{k}\left(t_{k}, \xi_{k}\right)\right| \leq C \delta_{k}\left(u+W_{k, t_{k}, \xi_{k}}\right) \text { in } M,
$$

and such that $\phi_{k}\left(t_{k}, \xi_{k}\right)$ is the unique solution of (6.1) in $K_{k, t_{k}, \xi_{k}}^{\perp}$ satisfying in addition (6.2). Also, for any $k$, the mapping $(t, \xi) \mapsto \phi_{k}(t, \xi) \in C^{1}(M)$ is continuous.

In Proposition 6.1, $K_{k, t_{k}, \xi_{k}}^{\perp}$ is again as in (2.22). The smallness assumption on $\eta$ and $\alpha$ is made clear in the course of the proof.

Proof. We let $C_{0}=C_{0}\left(n, g, u_{0}, h, f, \sigma, \pi, D\right)$ denote the smallest of the two positive constants appearing in equations (5.56) and (3.9), and we let:

$$
\varepsilon_{k}=4 C_{0} \delta_{k}
$$

Assume that $\eta$ in (2.11) is chosen so that there holds:

$$
C_{0} \eta \leq \frac{1}{4}
$$

Let $\left(t_{k}, \xi_{k}\right)_{k}$ be a sequence in $[1 / D, D] \times M$ and let $F_{k}=F\left(\varepsilon_{k}, t_{k}, \xi_{k}\right)$, where $F\left(\varepsilon_{k}, t_{k}, \xi_{k}\right)$ is defined in (2.27) and $\varepsilon_{k}$ is as in (6.3). For any $k$, we define the following mapping:

$$
\Psi_{k}:\left\{\begin{array}{l}
F\left(\varepsilon_{k}, t_{k}, \xi_{k}\right) \rightarrow F\left(\varepsilon_{k}, t_{k}, \xi_{k}\right) \cap\left\{\varphi \in K_{k, t_{k}, \xi_{k}}^{\perp},\|\varphi\|_{H^{1}(M)} \leq \frac{\varepsilon_{k}}{4}\right\} \\
v \longmapsto \phi_{k}=\phi_{k}\left(t_{k}, \xi_{k}, v\right)
\end{array}\right.
$$

where $\phi_{k}\left(t_{k}, \xi_{k}, v\right)$ is given by Proposition 3.3 . For any $k$, we endow the set $F_{k}$ with the norm

$$
\|v\|_{F_{k}}=\left\|\frac{v}{u+W_{k, t_{k}, \xi_{k}}}\right\|_{C^{0}(M)} .
$$

That the mapping $\Psi_{k}$ in (6.5) is well-defined for $k \geq k_{0}$ is a consequence of (6.3), (6.4), Proposition 5.3 and (3.9), which also show that the value of such a $k_{0}$ is independent of the choice of the sequence $\left(t_{k}, \xi_{k}\right)_{k}$. We now show that for $k$ sufficiently large the mapping $\Psi_{k}$ is a contraction for the norm (6.6).

Let, for any $k, v_{k}^{1}, v_{k}^{2} \in F_{k}$ and denote by $\phi_{k}^{1}$ and $\phi_{k}^{2}$ the associated images by $\Psi_{k}$. Using coherent notations, for $i=1,2$, we will let $u_{k}^{i}=u+W_{k, t_{k}, \xi_{k}}+\phi_{k}^{i}$ and $T_{k}^{i}$ will denote the unique solution of $\vec{\triangle}_{g} T_{k}^{i}=u_{k}^{i} 2^{*} X+Y$ in $M$. As before, we shall omit the dependence in $t_{k}$ and $\xi_{k}$ in the computations. 
By Proposition 3.3. for any $k$ there exist $\left(\lambda_{k, j}^{1}\right)_{0 \leq j \leq n}$ and $\left(\lambda_{k, j}^{2}\right)_{0 \leq j \leq n}$ such that $\phi_{k}^{1}-\phi_{k}^{2}$ satisfies:

$$
\begin{aligned}
\left(\triangle_{g}+h\right) & \left(\phi_{k}^{1}-\phi_{k}^{2}\right)=f\left[\left(u+W_{k}+\phi_{k}^{1}\right)^{2^{*}-1}-\left(u+W_{k}+\phi_{k}^{2}\right)^{2^{*}-1}\right] \\
& +\left(\pi^{2}+\left|\sigma+\mathcal{L}_{g} T\right|_{g}^{2}\right)\left[\left(u+W_{k}+\phi_{k}^{1}\right)^{-2^{*}-1}-\left(u+W_{k}+\phi_{k}^{2}\right)^{-2^{*}-1}\right] \\
& +\left(\left|\sigma+\mathcal{L}_{g} T_{k}^{1}\right|_{g}^{2}-\left|\sigma+\mathcal{L}_{g} T\right|_{g}^{2}\right)\left[\left(u+W_{k}+v_{k}^{1}\right)^{-2^{*}-1}-\left(u+W_{k}+v_{k}^{2}\right)^{-2^{*}-1}\right] \\
& +\left(u+W_{k}+v_{k}^{2}\right)^{-2^{*}-1}\left(\left|\sigma+\mathcal{L}_{g} T_{k}^{1}\right|_{g}^{2}-\left|\sigma+\mathcal{L}_{g} T_{k}^{2}\right|_{g}^{2}\right) \\
& +\sum_{j=0}^{n}\left(\lambda_{k, j}^{1}-\lambda_{k, j}^{2}\right)\left(\triangle_{g}+h\right) Z_{j, k} .
\end{aligned}
$$

We first estimate the $H^{1}$-norm of $\phi_{k}^{1}-\phi_{k}^{2}$. Since $v_{k}^{i} \in F_{k}$ there holds, with (2.11):

$$
\left|\vec{\triangle}_{g}\left(T_{k}^{1}-T_{k}^{2}\right)\right| \lesssim\left(u+W_{k}\right)^{2^{*}} \eta\left\|v_{k}^{1}-v_{k}^{2}\right\|_{F_{k}},
$$

so that mimicking the proof of Proposition 9.2 yields, with (2.24): for any $x \in M$,

$$
\left|\mathcal{L}_{g}\left(T_{k}^{1}-T_{k}^{2}\right)\right|(x) \lesssim \eta\left(\delta_{k}^{\frac{n-1}{2}} \theta_{k}(x)^{1-n}+1\right)\left\|v_{k}^{1}-v_{k}^{2}\right\|_{F_{k}} .
$$

We now apply $\left(\triangle_{g}+h\right)^{-1}$ to (6.7) and project on $K_{k}^{\perp}=K_{k, t_{k}, \xi_{k}}^{\perp}$. Using (9.18), Proposition 3.5 and the techniques developed in the proof of Proposition 3.3 we then get that:

$$
\left\|\phi_{k}^{1}-\phi_{k}^{2}\right\|_{H^{1}(M)} \lesssim \eta\left\|v_{k}^{1}-v_{k}^{2}\right\|_{F_{k}} .
$$

Let now $0 \leq j \leq n$ and integrate (6.7) against $Z_{j, k}$. With (6.9) one obtains:

$$
\sum_{j=0}^{n}\left|\lambda_{k, j}^{1}-\lambda_{k, j}^{2}\right| \lesssim \eta\left\|v_{k}^{1}-v_{k}^{2}\right\|_{F_{k}}
$$

We now show that, up to suitably choosing $\eta$ and $\alpha$ as in (2.11) and (2.25), for $k$ large enough there always holds:

$$
\left\|\phi_{k}^{1}-\phi_{k}^{2}\right\|_{F_{k}} \leq \frac{1}{2}\left\|v_{k}^{1}-v_{k}^{2}\right\|_{F_{k}} .
$$

For this, we let $\Phi_{k}=\phi_{k}^{1}-\phi_{k}^{2}$ and let $x_{k}$ be the point where the $F_{k}$-norm of $\Phi_{k}$ is attained:

$$
\left|\frac{\Phi_{k}}{u+W_{k}}\left(x_{k}\right)\right|=\sup _{x \in M}\left|\frac{\Phi_{k}}{u+W_{k}}(x)\right|=\left\|\Phi_{k}\right\|_{F_{k}} .
$$

We distinguish between two cases. We first assume that $d_{g_{\xi_{k}}}\left(\xi_{k}, x_{k}\right)=O\left(\delta_{k}\right)$. We then let, for any $x \in B_{0}\left(i_{g}(M) / \delta_{k}\right)$ :

$$
\tilde{\Phi}_{k}(x)=\frac{\delta_{k}^{\frac{n-2}{2}} \Phi_{k}}{\left\|\Phi_{k}\right\|_{F_{k}}+\left\|v_{k}^{1}-v_{k}^{2}\right\|_{F_{k}}}\left(\exp _{\xi_{k}}^{g_{\xi_{k}}}\left(\delta_{k} x\right)\right) .
$$

We also let $\tilde{x}_{k}=\frac{1}{\delta_{k}} \exp _{\xi_{k}}^{g_{\xi_{k}}-1}\left(x_{k}\right)$. There holds then: $\tilde{x}_{k} \rightarrow \tilde{x}_{0} \in \mathbb{R}^{n}$ as $k \rightarrow+\infty$ with $\left|\tilde{x}_{0}\right|=R_{0}$ for some $R_{0} \geq 0$. It is easily seen that $\left\|\tilde{\Phi}_{k}\right\|_{L^{\infty}} \leq 1$ and by (6.7), using Proposition 5.3 and standard elliptic theory, we get that the sequence $\tilde{\Phi}_{k}$ converges in $C_{l o c}^{1}\left(\mathbb{R}^{n}\right)$ to some function $\tilde{\Phi}_{0}$ which is a solution of:

$$
\triangle_{\xi} \tilde{\Phi}_{0}=\left(2^{*}-1\right) f\left(\xi_{0}\right) U_{\xi_{0}}^{2^{*}-2}+\sum_{j=0}^{n} \tilde{\lambda}_{0, j} \triangle_{\xi} V_{j, \xi_{0}},
$$


and which satisfies, for any $x \in \mathbb{R}^{n}$ :

$$
\left|\tilde{\Phi}_{0}(x)\right| \leq\left|\tilde{\Phi}_{0}\left(\tilde{x}_{0}\right)\right|=\left(\lim _{k \rightarrow+\infty} \frac{\left\|\Phi_{k}\right\|_{F_{k}}}{\left\|\Phi_{k}\right\|_{F_{k}}+\left\|v_{k}^{1}-v_{k}^{2}\right\|_{F_{k}}}\right)\left(1+\frac{f\left(\xi_{0}\right)}{n(n-2)} R_{0}^{2}\right)^{1-\frac{n}{2}} .
$$

In (6.14) $U_{\xi_{0}}$ and the $V_{j, \xi_{0}}$ are defined in (2.20) and (2.18), and we have let

$$
\tilde{\lambda}_{0, j}=\lim _{k \rightarrow+\infty} \frac{\lambda_{k, j}^{1}-\lambda_{k, j}^{2}}{\left\|\Phi_{k}\right\|_{F_{k}}+\left\|v_{k}^{1}-v_{k}^{2}\right\|_{F_{k}}} .
$$

This limit exists, up to a subsequence, by (6.10). A first thing to notice is that $\tilde{\Phi}_{0} \in L^{2^{*}}\left(\mathbb{R}^{n}\right)$. This is a consequence of (6.9), of the scaling invariance of the $L^{2^{*}}$ norm and of the definition of $\tilde{\Phi}_{k}$ in (6.13). Therefore, we can integrate (6.14) against $V_{j, \xi_{0}}$ for all $0 \leq j \leq n$ and (2.19) shows that there holds $\tilde{\lambda}_{0, j}=0$ for all $0 \leq j \leq n$. Since $\tilde{\Phi}_{0} \in L^{2^{*}}\left(\mathbb{R}^{n}\right)$, there holds then $\tilde{\Phi}_{0} \in H^{1}\left(\mathbb{R}^{n}\right)$ and the Bianchi-Egnell [5] classification result applies and shows that

$$
\tilde{\Phi}_{0} \in \operatorname{Span}\left\{V_{j, \xi_{0}}, 0 \leq j \leq n\right\} .
$$

Similarly to what we did in the proof of Claim 5.2, we will now show that $\tilde{\Phi}_{0} \in \operatorname{Span}\left\{V_{j, \xi_{0}}, 0 \leq j \leq\right.$ $n\}^{\perp}$. Since $\Phi_{k} \in K_{k}^{\perp}$ we can write, for any $R>0$ and $0 \leq j \leq n$, that

$$
\begin{aligned}
& \int_{B_{\xi_{k}}\left(R \delta_{k}\right)}\left\langle\nabla Z_{j, k}, \nabla \Phi_{k}\right\rangle_{g}+h Z_{j, k} \Phi_{k} d v_{g}=-\int_{\partial B_{\xi_{k}}\left(R \delta_{k}\right)} \Phi_{k} \partial_{\nu} Z_{j, k} d \sigma_{g} \\
& -\int_{M \backslash B_{\xi_{k}}\left(R \delta_{k}\right)}\left(h-c_{n} S_{g}\right) Z_{j, k} \Phi_{k} d v_{g}-\int_{M \backslash B_{\xi_{k}}\left(R \delta_{k}\right)}\left(\triangle_{g}+c_{n} S_{g}\right) Z_{j, k} \Phi_{k} d v_{g} .
\end{aligned}
$$

Mimicking the computations that led to (5.29) - (5.37) and using (9.2) and the definition of the $\|\cdot\|_{F_{k}}$-norm in (6.6) one obtains that, for any $0 \leq j \leq n$ :

$$
\left|\int_{B_{\xi_{k}}\left(R \delta_{k}\right)}\left\langle\nabla Z_{j, k}, \nabla \Phi_{k}\right\rangle_{g}+h Z_{j, k} \Phi_{k} d v_{g}\right| \lesssim\left(o(1)+R^{-2}\right)\left\|\Phi_{k}\right\|_{F_{k}} .
$$

Dividing both sides of (6.18) by $\left\|\Phi_{k}\right\|_{F_{k}}+\left\|v_{k}^{1}-v_{k}^{2}\right\|_{F_{k}}$, using the definition of $\tilde{\Phi}_{k}$ in (6.13) and the convergence of $\tilde{\Phi}_{k}$ to $\tilde{\Phi}_{0}$, letting $k \rightarrow+\infty$ and then $R \rightarrow+\infty$, we then obtain that $\tilde{\Phi}_{0} \in$ $\operatorname{Span}\left\{V_{j, \xi_{0}}, 0 \leq j \leq n\right\}^{\perp}$. With (6.16), this gives that $\tilde{\Phi}_{0} \equiv 0$. Using (6.15) this gives in turn that

$$
\left\|\phi_{k}^{1}-\phi_{k}^{2}\right\|_{F_{k}}=o\left(\left\|v_{k}^{1}-v_{k}^{2}\right\|_{F_{k}}\right)
$$

which proves (6.11) in this case.

We now assume that there holds, up to a subsequence, that

$$
\frac{d_{g_{\xi_{k}}}\left(\xi_{k}, x_{k}\right)}{\delta_{k}} \rightarrow+\infty
$$


as $k \rightarrow+\infty$, where $x_{k}$ is given by (6.12). Recall the definition of the operator $L_{u}$ introduced in (5.58). Then, using (5.56) and (6.3), we rewrite (6.7) as:

$$
\begin{aligned}
L_{u}\left(\Phi_{k}\right) & =O\left(\min \left(W_{k}^{2^{*}-2}, W_{k}\right)\left|\Phi_{k}\right|\right)+o\left(\left|\Phi_{k}\right|\right) \\
& +\left(\left|\sigma+\mathcal{L}_{g} T_{k}^{1}\right|_{g}^{2}-\left|\sigma+\mathcal{L}_{g} T\right|_{g}^{2}\right)\left[\left(u+W_{k}+v_{k}^{1}\right)^{-2^{*}-1}-\left(u+W_{k}+v_{k}^{2}\right)^{-2^{*}-1}\right] \\
& +\left(u+W_{k}+v_{k}^{2}\right)^{-2^{*}-1}\left(\left|\sigma+\mathcal{L}_{g} T_{k}^{1}\right|_{g}^{2}-\left|\sigma+\mathcal{L}_{g} T_{k}^{2}\right|_{g}^{2}\right) \\
& +\sum_{j=0}^{n}\left(\lambda_{k, j}^{1}-\lambda_{k, j}^{2}\right)\left(\triangle_{g}+h\right) Z_{j, k} .
\end{aligned}
$$

By (2.13), the Green's function $G_{u}$ of $L_{u}$ satisfies the pointwise bounds (5.60). We now write a Green representation formula for $L_{u}$ at $x_{k}$. Using (6.20), (6.8), (6.10), (9.11) below together with (2.24) and (5.60) we get that there holds:

$$
\begin{aligned}
\left|\Phi_{k}\left(x_{k}\right)\right| & \leq o\left(\left\|\Phi_{k}\right\|_{F_{k}}\left(u+W_{k}\right)\left(x_{k}\right)\right)+D_{0}(\alpha+\eta)\left\|v_{k}^{1}-v_{k}^{2}\right\|_{F_{k}} \\
& +\int_{M} G_{u}\left(x_{k}, y\right) \min \left(W_{k}^{2^{*}-2}(y), W_{k}(y)\right)\left|\Phi_{k}\right|(y) d v_{g}(y)
\end{aligned}
$$

for some positive $D_{0}$ that does not depend on $k$, and where $\eta$ and $\alpha$ are as in (2.11) and (2.25). Let $R>0$ be fixed. We have, using (6.19), that:

$$
\begin{aligned}
\mid \int_{M \backslash B_{\xi_{k}}\left(R \delta_{k}\right)} & G_{u}\left(x_{k}, y\right) \min \left(W_{k}^{2^{*}-2}(y), W_{k}(y)\right)\left|\Phi_{k}\right|(y) d v_{g}(y) \mid \\
& \lesssim \int_{M \backslash B_{\xi_{k}}\left(\sqrt{\delta_{k}}\right)} G_{u}\left(x_{k}, y\right) W_{k}(y)\left\|\Phi_{k}\right\|_{F_{k}} d v_{g}(y) \\
& +\int_{B_{\xi_{k}}\left(\sqrt{\delta_{k}}\right) \backslash B_{\xi_{k}}\left(R \delta_{k}\right)} G_{u}\left(x_{k}, y\right) W_{k}^{2^{*}-1}(y)\left\|\Phi_{k}\right\|_{F_{k}} d v_{g}(y) \\
& \leq D_{1}\left(\frac{1}{R^{2}}+\delta_{k}\right)\left(u+W_{k}\right)\left(x_{k}\right)\left\|\Phi_{k}\right\|_{F_{k}},
\end{aligned}
$$

for some positive constant $D_{1}$ which does not depend on $k$ or on $R$. Independently, if we let $p>\frac{n}{4}$ be fixed, two Hölder inequalities together with (6.19) and (6.9) show that

$$
\int_{B_{\xi_{k}}\left(R \delta_{k}\right)} G_{u}\left(x_{k}, y\right) W_{k}^{2^{*}-2}\left|\Phi_{k}(y)\right| d v_{g}(y) \lesssim D_{p} R^{\frac{n+2}{2}-\frac{n}{p}} \eta\left\|v_{k}^{1}-v_{k}^{2}\right\|_{F_{k}} \delta_{k}^{\frac{n-2}{2}} d_{g_{\xi_{k}}}\left(\xi_{k}, x_{k}\right)^{2-n}
$$

for some positive constant $D_{p}$ which depends on $p$ but not on $k$ or $R$. Choose now $R_{0}>0$ such that $D_{1} R_{0}^{-2}=\frac{1}{8}$, where $D_{1}$ is given by (6.22). Assume then that $\alpha$ and $\eta$ are small enough to have $D_{p} R_{0} \frac{n+2}{2}-\frac{n}{p} \eta \leq \frac{1}{8}$ and $D_{0}(\alpha+\eta) \leq \frac{1}{8}$, where $D_{0}$ and $D_{p}$ are given by (6.21) and (6.23). Then, plugging (6.22) and (6.23) in (6.21) and using the definition (6.12) of $x_{k}$ gives:

$$
\left\|\phi_{k}^{1}-\phi_{k}^{2}\right\|_{F_{k}} \leq \frac{1}{2}\left\|v_{k}^{1}-v_{k}^{2}\right\|_{F_{k}}
$$

thus concluding the proof of (6.11). The fact that (6.11) holds for large $k$ independent on the choice of $\left(t_{k}, \xi_{k}\right)_{k}$ follows by a standard contradiction argument, up to passing to a subsequence.

Now, the uniqueness property that defines $\phi\left(t_{k}, \xi_{k}, v_{k}\right)$ (and is stated in Proposition 3.3) shows that, for any $k$ large enough, a function $\varphi \in F\left(\varepsilon_{k}, t_{k}, \xi_{k}\right) \cap\left\{\varphi \in K_{k, t_{k}, \xi_{k}}^{\perp},\|\varphi\|_{H^{1}(M)} \leq \frac{\varepsilon_{k}}{4}\right\}$ solves (6.1) 
if and only if it is a fixed-point of $\Psi_{k}$ defined in (6.5). Using (6.11), Banach-Picard's fixed-point theorem asserts, for any $k$, the existence of such a fixed-point $\phi_{k}\left(t_{k}, \xi_{k}\right)$ as well as its uniqueness in $F\left(C \delta_{k}, t_{k}, \xi_{k}\right) \cap K_{k, t_{k}, \xi_{k}}^{\perp} \cap B_{H^{1}(M)}\left(0, C \delta_{k}\right)$. The estimates in (6.2) follow then from (3.9), (5.3) and (6.3). Finally, the continuity of the mapping $(t, \xi) \in(0,+\infty) \times M \mapsto \phi_{k}(t, \xi) \in C^{1}(M)$ follows from direct arguments, using (4.5), standard elliptic theory and the uniqueness property of $\phi_{k}(t, \xi)$ in $F\left(C \delta_{k}, t_{k}, \xi_{k}\right) \cap K_{k, t_{k}, \xi_{k}}^{\perp} \cap B_{H^{1}(M)}\left(0, C \delta_{k}\right)$, thus concluding the proof of Proposition 6.1 .

One might surprised by the use of Banach-Picard's fixed-point theorem in the proof of Proposition 6.1 In view of Proposition 5.3. an application of Schauder's fixed-point theorem would seem preferable to construct a solution of (6.1) - and the proof would indeed be simpler. The reason for using Banach-Picard's theorem is that Schauder's theorem does not provide a preferred solution of (6.1), and in particular yields no uniqueness property on the remainder $\phi_{k}(t, \xi)$ thus constructed. A striking consequence is that if $\phi_{k}(t, \xi)$ is not uniquely determined in some way, one is not able to prove its continuity in the choice of $(t, \xi) \in(0,+\infty) \times M$.

This remark is best understood anticipating a little on Sections 7 and 8 . If we were to construct a remainder $\phi_{k}(t, \xi)$ by Schauder's fixed-point theorem it would still satisfy (6.2) and the (analogue of the) estimates of Proposition [5.1 so that the expansions we perform in Section 7 would still hold true. In particular, one would obtain an exact analogue of (8.2), but where the error terms $R_{k}^{i}(t, p)$, $0 \leq i \leq n$, would only be bounded functions whose uniform bound goes to zero as $k \rightarrow+\infty$. Since $(t, \xi) \mapsto \phi_{k}(t, \xi)$ is not continuous, these $R_{k}^{i}(t, p)$ would be non-continuous too, and the usual final annihilating arguments would fail.

The pointwise estimates obtained in Proposition 5.3 still hold true for the function $\phi_{k}(t, \xi)$ given by Proposition 6.1 when substituting $\varepsilon_{k}$ by $\mu_{k}$. Estimate (9.11) also remains true for the field of 1-forms $T_{k, t, \xi}$ associated to $u+W_{k, t, \xi}+\phi_{k}(t, \xi)$ by (3.3). Proposition 5.1 gives the following control:

Proposition 6.2. Let $D>0,\left(t_{k}, \xi_{k}\right)_{k}$ be a sequence in $[1 / D, D] \times M$, and let $\phi_{k}=\phi_{k}\left(t_{k}, \xi_{k}\right)$ denote the function given by Proposition 6.1. Let $\left(x_{k}\right)_{k}$ be any sequence of points in $B_{\xi_{k}}\left(2 \sqrt{\delta_{k}}\right)$. Then there holds:

- If $n \geq 7$ :

$$
\begin{aligned}
\left|\phi_{k}\left(x_{k}\right)\right| & \lesssim \delta_{k}+\delta_{k}\|\nabla f\|_{L^{\infty}\left(2 r_{k}\right)}+\delta_{k}\left\|h-c_{n} S_{g}\right\|_{L^{\infty}\left(2 r_{k}\right)}+\delta_{k} \\
& +\left(\frac{\delta_{k}}{\theta_{k}\left(x_{k}\right)}\right)^{2}+\left[\delta_{k}^{\frac{n}{2}}+\delta_{k}\|\nabla f\|_{L^{\infty}\left(2 r_{k}\right)}\right. \\
& \left.+\left\|h-c_{n} S_{g}\right\|_{L^{\infty}\left(2 r_{k}\right)}\left(\theta_{k}\left(x_{k}\right)^{2}+\delta_{k}^{2} \ln \left(\frac{\theta_{k}\left(x_{k}\right)}{\delta_{k}}\right)\right)+\theta_{k}\left(x_{k}\right)^{4} \mathbb{1}_{n l c f}\right] W_{k}\left(x_{k}\right) .
\end{aligned}
$$

- If $n=6$ :

$$
\begin{aligned}
\left|\phi_{k}\left(x_{k}\right)\right| & \lesssim \delta_{k}+\delta_{k}\|\nabla f\|_{L^{\infty}\left(2 r_{k}\right)}+\delta_{k}\left\|h-\frac{1}{5} S_{g}-2 f u\right\|_{L^{\infty}\left(2 r_{k}\right)} \\
& +\left[\delta_{k}^{3}+\delta_{k}\|\nabla f\|_{L^{\infty}\left(2 r_{k}\right)}+\left\|h-\frac{1}{5} S_{g}-2 f u\right\|_{L^{\infty}\left(2 r_{k}\right)}\left(\theta_{k}\left(x_{k}\right)^{2}+\delta_{k}^{2} \ln \left(\frac{\theta_{k}\left(x_{k}\right)}{\delta_{k}}\right)\right)\right] W_{k}\left(x_{k}\right) .
\end{aligned}
$$

Proof. Proposition 5.3 shows that there holds:

$$
\left\|\phi_{k}\right\|_{L^{\infty}\left(B_{\xi_{k}}\left(2 r_{k}\right) \backslash B_{\xi_{k}}\left(\sqrt{\delta_{k}}\right)\right)}+\sqrt{\delta_{k}}\left\|\nabla \phi_{k}\right\|_{L^{\infty}\left(B_{\xi_{k}}\left(2 r_{k}\right) \backslash B_{\xi_{k}}\left(\sqrt{\delta_{k}}\right)\right)} \lesssim \delta_{k},
$$

and the result then follows from an application of Proposition 5.1 . 
To be able to perform the asymptotic expansion along $K_{k, t, \xi}^{\perp}$ in the next section, we will need more precise estimates than (5.56) on the behaviour of $\phi_{k}(t, \xi)$ at distances from $\xi_{k}$ which are large compared to $\sqrt{\mu_{k}}$. We quantify more precisely the fall-off of $\phi_{k}(t, \xi)$ far away from the center of the bubbles in the next Proposition:

Proposition 6.3. Let $D>0,\left(t_{k}, \xi_{k}\right)_{k}$ be a sequence in $[1 / D, D] \times M$, and let $\phi_{k}=\phi_{k}\left(t_{k}, \xi_{k}\right)$ denote the function given by Proposition [6.1. Let $\left(R_{k}\right)_{k}, R_{k} \geq 1$, denote a sequence of positive numbers. There holds:

$$
\left\|\phi_{k}\right\|_{L^{\infty}\left(M \backslash B_{\xi_{k}}\left(R_{k} \sqrt{\delta_{k}}\right)\right)} \lesssim \frac{\delta_{k}}{R_{k}^{2}}+R_{k}^{2} \delta_{k}^{2}+\delta_{k}^{\frac{n-2}{2}} r_{k}^{-n} .
$$

Proof. Let $\left(t_{k}, \xi_{k}\right)_{k}$ be a sequence in $[1 / D, D] \times M$ and let $\left(R_{k}\right)_{k}, R_{k} \geq 1$, denote a sequence of positive numbers. Let $\left(x_{k}\right)_{k}$ be a sequence of points in $M$ satisfying $d_{g_{\xi_{k}}}\left(\xi_{k}, x_{k}\right) \geq R_{k} \sqrt{\delta_{k}}$. Let again $G_{u}$ denote the Green's function of the operator $L_{u}$ defined in (5.58). We use (5.68) and (9.18) below to write that there holds, for some positive constant $C$ that neither depends on $k$ nor on $\eta$ as in (2.11), that:

$$
\begin{array}{r}
\int_{M} G_{u}\left(x_{k}, y\right) \frac{\left|\mathcal{L}_{g} T_{k}+\sigma\right|_{g}^{2}-\left|\mathcal{L}_{g} T+\sigma\right|_{g}^{2}}{\left(u+W_{k}+v_{k}\right)^{2^{*}+1}}(y) d v_{g}(y) \leq C\left(\delta_{k}^{\frac{n}{2}} \theta_{k}\left(x_{k}\right)^{2-n}\right. \\
\left.+\eta\left\|\phi_{k}\right\|_{L^{\infty}\left(M \backslash B_{\xi_{k}}\left(R_{k} \sqrt{\delta_{k}}\right)\right)}+\delta_{k}^{2}+\eta R_{k}^{2} \delta_{k}^{2}+\delta_{k}^{\frac{n-1}{2}} \theta_{k}\left(x_{k}\right)^{3-n}\right) .
\end{array}
$$

Here, $T_{k}=T_{k, t_{k}, \xi_{k}}$ is the solution of the 1 -form equation in (6.1). Assume now that $\eta$ in (2.11) is small enough so as to have

$$
C \eta \leq \frac{1}{2}
$$

where $C$ is the constant appearing in (6.25). Estimate (6.24) then follows from (6.25) by choosing the sequence $\left(x_{k}\right)_{k}$ to be such that

$$
\left|\phi_{k}\left(x_{k}\right)\right|=\left\|\phi_{k}\right\|_{L^{\infty}\left(M \backslash B_{\xi_{k}}\left(R_{k} \sqrt{\delta_{k}}\right)\right)}
$$

and by writing a representation formula for $L_{u}$ at $x_{k}$. The representation formula is written for (5.59) and the terms appearing in it are estimated by using (5.61)-(5.67) and (6.2).

The considerations in the remark following Proposition 5.3 apply here too: Propositions 6.1, 6.2 and 6.3 still hold true for arbitrary choices of $h, \pi \in C^{0}(M), f \in C^{1}(M), \sigma \in C^{0}(M)$ and $Y \in C^{0}(M)$ once $X$ is given by (2.24).

\section{Expansion of the KeRnel COEFFicients}

Let $D>0$. We let, for any $p \in \overline{B_{0}(1)}$ :

$$
y_{k}=\exp _{\xi_{k}}^{g_{\xi_{k}}}\left(\beta_{k} p\right),
$$

where $\beta_{k}$ is defined in (2.6) and $\left(\xi_{k}\right)_{k}$ is the sequence chosen in Section 2. Throughout this section the functions $W_{k, t, y_{k}}$ and $Z_{i, k, t, y_{k}}$ defined in (2.17) and (2.21) will be denoted by $W_{k, t, p}$ and $Z_{i, k, t, p}$, with $\delta_{k}(t)$ again given by (2.16). For any $(t, p) \in[1 / D, D] \times \overline{B_{0}(1)}$ we will denote by $\phi_{k}(t, p)$ the function $\phi_{k}\left(t, y_{k}\right)$ given by Proposition 6.1 when $y_{k}$ is given by (7.1), and as before we let $u_{k, t, p}=$ $u+W_{k, t, p}+\phi_{k}(t, p)$. Proposition 6.1 shows that there exist real numbers $\left(\lambda_{k}^{i}(t, p)\right)_{0 \leq i \leq n}$ such that $u_{k, t, p}$ satisfies:

$$
\left\{\begin{array}{l}
\left(\triangle_{g}+h\right) u_{k, t, p}=f u_{k, t, p}^{2^{*}-1}+\frac{\left|\mathcal{L}_{g} T_{k, t, p}+\sigma\right|_{g}^{2}+\pi^{2}}{u_{k, t, p}^{2^{*}+1}}+\sum_{i=0}^{n} \lambda_{k}^{i}(t, p)\left(\triangle_{g}+h\right) Z_{i, k, t, p}, \\
\vec{\triangle}_{g} T_{k, t, p}=u_{k, t, p}^{2^{*}} X+Y .
\end{array}\right.
$$


Since $\phi_{k}(t, p), W_{k, t, p}$ and $Z_{i, k, t, p}$ are continuous in the choice of $(t, p)$, then so are the $\left(\lambda_{k}^{i}(t, p)\right)_{0 \leq i \leq n}$.

In this section we conclude the proof of Theorem 1.1 by showing that for any $k$ there exists $\left(t_{k}, p_{k}\right)$ such that $\lambda_{k}^{i}\left(t_{k}, p_{k}\right)=0$ for any $0 \leq i \leq n$. By (7.2), the function $u_{k, t_{k}, p_{k}}$ will therefore provide the desired solution of (1.1) and this will conclude the proof of Theorem 1.1. We do this by performing an asymptotic expansion of the $\left(\lambda_{k}^{i}(t, p)\right)_{0 \leq i \leq n}$ as $k \rightarrow+\infty$ towards some limiting function that possesses zeroes. As before, we distinguish between the $n=6$ and $n \geq 7$ cases. All the expansions obtained in this section will be uniform in the choice of $(t, p) \in[1 / D, D] \times \overline{B_{0}(1)}$. In this section we will often apply previously obtained results, such as Proposition [6.1. They will always be applied for the sequence $\left(t, y_{k}\right)$ where $y_{k}$ is given by (7.1). As mentioned in the Introduction, the expansions in this section do not rely on the assumption $r_{k} \rightarrow 0$.

7.1. The $n \geq 7$ case. We start by re-writing the scalar equation in (7.2) as:

$$
\begin{aligned}
& \sum_{i=0}^{n} \lambda_{k}^{i}(t, p)\left(\triangle_{g}+h\right) Z_{i, k, t, p}= \\
& \left(\triangle_{g}+h\right) W_{k, t, p}-f\left(y_{k}\right) W_{k, t, p}^{2^{*}-1}+\left(f\left(y_{k}\right)-f\right) W_{k, t, p}^{2^{*}-1} \\
& -f\left[\left(u+W_{k, t, p}+\phi_{k}(t, p)\right)^{2^{*}-1}-\left(u+W_{k, t, p}\right)^{2^{*}-1}-\left(2^{*}-1\right)\left(u+W_{k, t, p}\right)^{2^{*}-2} \phi_{k}(t, p)\right] \\
& -f\left[\left(u+W_{k, t, p}\right)^{2^{*}-1}-u^{2^{*}-1}-W_{k, t, p}^{2^{*}-1}\right] \\
& +\left(\triangle_{g}+h\right) \phi_{k}(t, p)-\left(2^{*}-1\right) f\left(y_{k}\right) W_{k, t, p}^{2^{*}-2} \phi_{k}(t, p) \\
& -\left(2^{*}-1\right) f\left[\left(u+W_{k, t, p}\right)^{2^{*}-2}-W_{k, t, p}^{2^{*}-2}\right] \phi_{k}(t, p) \\
& +\left(2^{*}-1\right)\left(f\left(y_{k}\right)-f\right) W_{k, t, p}^{2^{*}-2} \phi_{k}(t, p) \\
& +\frac{\left|\mathcal{L}_{g} T+\sigma\right|_{g}^{2}+\pi^{2}}{u^{2^{*}+1}}-\frac{\left|\mathcal{L}_{g} T_{k, t, p}+\sigma\right|_{g}^{2}+\pi^{2}}{\left(u+W_{k, t, p}+\phi_{k}(t, p)\right)^{2^{*}+1}} .
\end{aligned}
$$


We integrate each side of equation (7.3) against $Z_{i, k, t, p}$, for a given $0 \leq i \leq n$. For any $0 \leq i \leq n$ we let:

$$
\begin{aligned}
& I_{1, i}= \int_{M}\left(\left(\triangle_{g}+h\right) W_{k, t, p}-f\left(y_{k}\right) W_{k, t, p}^{2^{*}-1}+\left(f\left(y_{k}\right)-f\right) W_{k, t, p}^{2^{*}-1}\right) Z_{i, k, t, p} d v_{g}, \\
& I_{2, i}=-\int_{M} f\left[\left(u+W_{k, t, p}+\phi_{k}(t, p)\right)^{2^{*}-1}-\left(u+W_{k, t, p}\right)^{2^{*}-1}\right. \\
&\left.-\left(2^{*}-1\right)\left(u+W_{k, t, p}\right)^{2^{*}-2} \phi_{k}(t, p)\right] Z_{i, k, t, p} d v_{g}, \\
& I_{3, i}=-\int_{M} f\left[\left(u+W_{k, t, p}\right)^{2^{*}-1}-u^{2^{*}-1}-W_{k, t, p}^{2^{*}-1}\right] Z_{i, k, t, p} d v_{g}, \\
& I_{4, i}=\int_{M}\left[\left(\triangle_{g}+h\right) \phi_{k}(t, \xi)-\left(2^{*}-1\right) f\left(\xi_{k}\right) W_{k, t, \xi}^{2^{*}-2} \phi_{k}(t, \xi)\right] Z_{i, k, t, \xi} d v_{g} \\
& I_{5, i}=-\left(2^{*}-1\right) \int_{M} f\left[\left(u+W_{k, t, p}\right)^{2^{*}-2}-W_{k, t, p}^{2^{*}-2}\right] \phi_{k}(t, p) Z_{i, k, t, p} d v_{g}, \\
& I_{6, i}=\left(2^{*}-1\right) \int_{M}\left(f\left(y_{k}\right)-f\right) W_{k, t, p}^{2^{*}-2} \phi_{k}(t, p) Z_{i, k, t, p} d v_{g}, \\
& I_{7, i}=\int_{M}\left(\frac{\left|\mathcal{L}_{g} T+\sigma\right|_{g}^{2}+\pi^{2}}{u^{2^{*}+1}}-\frac{\left|\mathcal{L}_{g} T_{k, t, p}+\sigma\right|_{g}^{2}+\pi^{2}}{\left(u+W_{k, t, p}+\phi_{k}(t, p)\right)^{2^{*}+1}}\right) Z_{i, k, t, p} d v_{g} .
\end{aligned}
$$

We will need precise asymptotic expansions of the $\lambda_{k}^{i}(t, p)$ and so will always distinguish in the following between the $i=0$ and $1 \leq i \leq n$ cases, to take into account the different decay of the $Z_{i, k, t, p}$. Our aim is to show that the dominant contributions in the expansion of the $\lambda_{k}^{i}(t, p)$ come from the integrals $I_{1, i}$ and $I_{3, i}$ in (7.4).

The integrals in (7.4) are computed in a series of Claims. We start with the computations of $I_{1, i}$ :

Claim 7.1. There holds:

$$
\begin{aligned}
I_{1,0}= & \frac{8(n-1)}{(n-2)(n-4)} K_{n}^{-n} f\left(\xi_{0}\right)^{-\frac{n}{2}} \tau_{k} \mu_{k}^{2} H(p) t^{2} \\
& -\frac{1}{3} \frac{n(n-2)}{(n-4)(n-6)} f\left(\xi_{0}\right)^{-1-\frac{n}{2}} K_{n}^{-n}\left|W_{g}\left(\xi_{0}\right)\right|_{g}^{2} \mu_{k}^{4} t^{4}+o\left(\tau_{k} \delta_{k}^{2}\right)+o\left(\delta_{k}^{4} \mathbb{1}_{n l c f}\right)+o\left(\delta_{k}^{\frac{n-2}{2}}\right),
\end{aligned}
$$

and, for any $1 \leq i \leq n$ :

$$
\begin{aligned}
I_{1, i}= & \frac{2 n(n-1)}{n-4} K_{n}^{-n} f\left(\xi_{0}\right)^{-\frac{n}{2}} \frac{\tau_{k}}{\beta_{k}} \mu_{k}^{3} \nabla_{i} H(p) t^{3} \\
& -f\left(\xi_{0}\right)^{-1-\frac{n}{2}} K_{n}^{-n} \frac{n^{2}(n-2)^{2}}{24(n-4)(n-6)} \nabla_{i}\left(|W(\cdot)|_{g}^{2}\right)\left(\xi_{0}\right) \mu_{k}^{5} t^{5}+o\left(\delta_{k}^{5} \mathbb{1}_{n l c f}\right)+o\left(\delta_{k}^{\frac{n}{2}}\right) .
\end{aligned}
$$

Here $\xi_{0}=\lim _{k \rightarrow+\infty} \xi_{k}$ and $\tau_{k}$ and $\mu_{k}$ are as in (2.5).

In (7.5) and (7.6) $W_{g}\left(\xi_{0}\right)$ denotes the Weyl tensor at $\xi_{0}$ (which vanishes if $(M, g)$ is locally conformally flat) and $K_{n}^{-n}$ is the $H^{1}$-energy of the standard bubble defined by:

$$
K_{n}=\sqrt{\frac{4}{n(n-2) \omega_{n}^{\frac{2}{n}}}},
$$

where $\omega_{n}$ is the volume of the standard unit $n$-sphere. 
Proof. Using the definition of conformal normal coordinates as in (2.1) and (2.3), and using (2.17) and (2.21), there holds, for any fixed $t>0$ and $\xi \in M$, that:

$$
\begin{aligned}
\frac{\partial}{\partial t} W_{k, \delta, \xi} & =\frac{n-2}{2 t} Z_{0, k, t, \xi}, \\
\frac{\partial}{\partial \xi_{i}} W_{k, t, \xi} & =\frac{f(\xi)}{n \delta_{k}(t)} Z_{i, k, t, \xi}+O\left(\delta_{k}^{\frac{n-2}{2}} r_{k}^{1-n} \mathbb{1}_{r_{k} \leq d_{g_{\xi}} \leq 2 r_{k}}\right)+O\left(|\nabla f(\xi)|_{g} W_{k, t, \xi}\right)+O\left(d_{g_{\xi}}(\xi, \cdot) W_{k, t, \xi}\right) .
\end{aligned}
$$

These expressions are for instance obtained from the estimations in Appendix A in Esposito-PistoiaVétois [23] and those in Section 4 of Robert-Vétois [45. Estimates (7.5) and (7.6) follow then from straightforward computations using (2.7), (2.23) and (2.15).

Claim 7.2. There holds:

$$
\begin{aligned}
& I_{2,0}=o\left(\delta_{k}^{\frac{n-2}{2}}\right) \text { if }(M, g) \text { is l.c.f. or if } n \leq 10, \\
& I_{2,0}=o\left(\delta_{k}^{4}\right) \text { if }(M, g) \text { is not l.c.f. and } n \geq 11,
\end{aligned}
$$

and, for $1 \leq i \leq n$,

$$
I_{2, i}=o\left(\delta_{k}^{\frac{n}{2}}\right)+o\left(\delta_{k}^{5} \mathbb{1}_{n l c f}\right) .
$$

Proof. First, we have that:

$$
\begin{array}{r}
f\left[\left(u+W_{k, t, p}+\phi_{k}(t, p)\right)^{2^{*}-1}-\left(u+W_{k, t, p}\right)^{2^{*}-1}-\left(2^{*}-1\right)\left(u+W_{k, t, p}\right)^{2^{*}-2} \phi_{k}(t, p)\right] \\
\lesssim\left(u+W_{k, t, p}\right)^{2^{*}-3}\left|\phi_{k}(t, p)\right|^{2} .
\end{array}
$$

Using (6.2) and since $n \geq 7$ and $\left|Z_{0, k, t, p}\right| \lesssim W_{k, t, p}$, we write that:

$$
\begin{aligned}
\int_{M \backslash B_{y_{k}}\left(\sqrt{\delta_{k}}\right)}\left(u+W_{k, t, p}\right)^{2^{*}-3}\left|\phi_{k}(t, p)\right|^{2}\left|Z_{0, k, t, p}\right| d v_{g} \lesssim & \delta_{k}^{2} \int_{B_{y_{k}}\left(2 r_{k}\right) \backslash B_{y_{k}}\left(\sqrt{\delta_{k}}\right)}\left|Z_{0, k, t, p}\right| d v_{g} \\
& +\delta_{k}^{2} \int_{B_{y_{k}}\left(2 r_{k}\right) \backslash B_{y_{k}}\left(\sqrt{\delta_{k}}\right)} W_{k, t, p}^{2^{*}} d v_{g}
\end{aligned}
$$

which gives then

$$
\int_{M \backslash B_{y_{k}}\left(\sqrt{\delta_{k}}\right)}\left(u+W_{k, t, p}\right)^{2^{*}-3}\left|\phi_{k}(t, p)\right|^{2}\left|Z_{0, k, t, p}\right| d v_{g}=o\left(\delta_{k}^{\frac{n}{2}}\right) .
$$

We now use Proposition 6.2 and (2.23) to write that there holds:

$$
\int_{B_{y_{k}}\left(\sqrt{\delta_{k}}\right)} W_{k, t, p}^{2^{*}-3}\left|\phi_{k}(t, p)\right|^{2}\left|Z_{0, k, t, p}\right| d v_{g} \lesssim o\left(\delta_{k}^{\frac{n}{2}}\right)+o\left(\delta_{k}^{4} \mathbb{1}_{n l c f}\right)+\delta_{k}^{4}\left\|h-c_{n} S_{g}\right\|_{L^{\infty}\left(2 r_{k}\right)}^{2}
$$

Assume first that $(M, g)$ is locally conformally flat or that $n \leq 10$. Then, using (2.7) there holds $\left\|h-c_{n} S_{g}\right\|_{L^{\infty}\left(2 r_{k}\right)}^{2}=\delta_{k}^{n-6}$ so that with (7.11) we obtain (7.8). Assume then that $n \geq 11$ and that $(M, g)$ is not locally conformally flat. Then (2.7) shows that $\left\|h-c_{n} S_{g}\right\|_{L^{\infty}\left(2 r_{k}\right)}^{2}=\delta_{k}^{4}$ so that gathering (7.11) and (7.12) and since $n \geq 7$, we obtain (7.9).

Let now $1 \leq i \leq n$ be fixed. On one side, using (6.2) and since $n \geq 7$ and $\left|Z_{i, k, t, p}\right| \lesssim W_{k, t, p}$ we have that

$$
\int_{M \backslash B_{y_{k}}\left(\sqrt{\delta_{k}}\right)}\left(u+W_{k, t, p}\right)^{2^{*}-3}\left|\phi_{k}(t, p)\right|^{2}\left|Z_{i, k, t, p}\right| d v_{g}=o\left(\delta_{k}^{\frac{n}{2}}\right) .
$$


On the other side, using again Proposition 6.2, (2.23) and (2.7) shows that

$$
\int_{B_{y_{k}}\left(\sqrt{\delta_{k}}\right)} W_{k, t, p}^{2^{*}-3}\left|\phi_{k}(t, p)\right|^{2}\left|Z_{i, k, t, p}\right| d v_{g}=o\left(\delta_{k}^{\frac{n}{2}}\right)+o\left(\delta_{k}^{5} \mathbb{1}_{n l c f}\right) .
$$

Combining the latter estimates we get (7.10).

Claim 7.3. There holds:

$$
I_{3,0}=-\frac{1}{2}(n-2)^{2}(n(n-2))^{\frac{n-2}{2}} \omega_{n-1} f\left(\xi_{0}\right)^{1-\frac{n}{2}} u\left(\xi_{0}\right) \delta_{k}^{\frac{n-2}{2}}+o\left(\delta_{k}^{\frac{n-2}{2}}\right)
$$

and, for any $1 \leq i \leq n$,

$$
I_{3, i}=-C(n) f\left(\xi_{0}\right)^{-\frac{n}{2}} \nabla_{i} u\left(\xi_{0}\right) \delta_{k}^{\frac{n}{2}}+o\left(\delta_{k}^{\frac{n}{2}}\right),
$$

where $C(n)$ is some explicit, positive, numerical constant only depending on $n$.

Proof. Write that:

$$
\begin{aligned}
\left|\int_{M \backslash B_{y_{k}}\left(\sqrt{\delta_{k}}\right)} f\left[\left(u+W_{k, t, p}\right)^{2^{*}-1}-u^{2^{*}-1}-W_{k, t, p}^{2^{*}-1}\right] Z_{i, k, t, p} d v_{g}\right| & \lesssim \int_{M \backslash B_{y_{k}}\left(\sqrt{\delta_{k}}\right)} W_{k, t, p}\left|Z_{i, k, t, p}\right| d v_{g} \\
& \lesssim\left\{\begin{array}{l}
o\left(\delta_{k}^{\frac{n-2}{2}}\right) \text { if } i=0, \\
o\left(\delta_{k}^{\frac{n}{2}}\right) \text { if } 1 \leq i \leq n .
\end{array}\right.
\end{aligned}
$$

The integral over $B_{y_{k}}\left(\sqrt{\delta_{k}}\right)$ is then easily computed using the explicit expressions of $W_{k, t, p}$ and $Z_{i, k, t, p}$ given in (2.17) and (2.21). Straightforward computations give (7.13) and (7.14).

Claim 7.4. There holds:

$$
I_{4,0}=o\left(\delta_{k}^{\frac{n-2}{2}}\right)+o\left(\delta_{k}^{4} \mathbb{1}_{n l c f}\right)
$$

and, for any $1 \leq i \leq n$ :

$$
I_{4, i}=o\left(\delta_{k}^{\frac{n}{2}}\right)+o\left(\delta_{k}^{5} \mathbb{1}_{n l c f}\right) .
$$

Proof. Integrating by parts, $I_{4, i}$ is best written as:

$$
I_{4, i}=\int_{M}\left[\left(\triangle_{g}+h\right) Z_{i, k, t, p}-\left(2^{*}-1\right) f\left(y_{k}\right) W_{k, t, p}^{2^{*}-2} Z_{i, k, t, p}\right] \phi_{k}(t, p) d v_{g} .
$$

Again, we start with $I_{4,0}$. Using (6.2), there holds:

$$
\int_{M}\left(\delta_{k}^{\frac{n-2}{2}} r_{k}^{-n} \mathbb{1}_{r_{k} \leq d_{g_{y_{k}}} \leq 2 r_{k}}+\delta_{k}^{\frac{n-2}{2}} \mathbb{1}_{n l c f}\right)\left|\phi_{k}(t, \xi)\right| d v_{g}=o\left(\delta_{k}^{\frac{n-2}{2}}\right) .
$$

Using (2.7) and (6.2) it is easily seen that there also holds:

$$
\int_{M}\left(h-c_{n} S_{g}\right) Z_{0, k, t, p} \phi_{k}(t, p) d v_{g}=o\left(\delta_{k}^{\frac{n-2}{2}}\right)+o\left(\delta_{k}^{4} \mathbb{1}_{n l c f}\right) .
$$

Also, (2.4) shows that $\left|S_{g_{y_{k}}}\right|=O\left(d_{g_{y_{k}}}\left(y_{k}, \cdot\right)^{2}\right)$, so that with (6.2) we obtain:

$$
\int_{M} \Lambda_{y_{k}}^{2^{*}-2} c_{n} S_{g_{y_{k}}} Z_{0, k, t, p} \phi_{k}(t, p)=o\left(\delta_{k}^{\frac{n-2}{2}}\right)+o\left(\delta_{k}^{4} \mathbb{1}_{n l c f}\right) .
$$

Combining (9.2) with (7.17) yields in the end (7.15). 
Let now $1 \leq i \leq n$. Because of (2.15) and (6.2) we have:

$$
\int_{M} \delta_{k}^{\frac{n}{2}} r_{k}^{-n-1} \mathbb{1}_{r_{k} \leq d_{g_{y_{k}}} \leq 2 r_{k}}\left|\phi_{k}(t, p)\right| d v_{g}=o\left(\delta_{k}^{\frac{n}{2}}\right) .
$$

There holds that

$$
\begin{aligned}
\int_{M} \mid & \left(h-c_{n} S_{g}\right) Z_{i, k, t, p} \phi_{k}(t, p) \mid d v_{g} \\
= & \int_{M \backslash B_{y_{k}}\left(\sqrt{\delta_{k}}\right)}\left|\left(h-c_{n} S_{g}\right) Z_{i, k, t, p} \phi_{k}(t, p)\right| d v_{g}+\int_{B_{y_{k}}\left(\sqrt{\delta_{k}}\right)}\left|\left(h-c_{n} S_{g}\right) Z_{i, k, t, p} \phi_{k}(t, p)\right| d v_{g} \\
= & o\left(\delta_{k}^{\frac{n}{2}}\right)+o\left(\delta_{k}^{5} \mathbb{1}_{n l c f}\right),
\end{aligned}
$$

where the first integral is estimated using (6.2) and the second one is estimated using Proposition 6.2 and (2.7). Assume that $(M, g)$ is not locally conformally flat. Write again that:

$$
\begin{aligned}
\int_{M} c_{n} & \Lambda_{y_{k}} S_{g_{y_{k}}} Z_{i, k, t, p} \phi_{k}(t, p) d v_{g} \\
& =\int_{M \backslash B_{y_{k}}\left(\sqrt{\delta_{k}}\right)} c_{n} \Lambda_{y_{k}} S_{g_{y_{k}}} Z_{i, k, t, p} \phi_{k}(t, p) d v_{g}+\int_{B_{y_{k}}\left(\sqrt{\delta_{k}}\right)} c_{n} \Lambda_{y_{k}} S_{g_{y_{k}}} Z_{i, k, t, p} \phi_{k}(t, p) d v_{g} \\
& =o\left(\delta_{k}^{\frac{n}{2}}\right)+o\left(\delta_{k}^{5} \mathbb{1}_{n l c f}\right),
\end{aligned}
$$

where as before the first integral is estimated using (6.2) and the second one using Proposition 6.2, (2.7) and (2.23). Note, as a simple computation shows, that (6.2) alone would not be enough to estimate the integral over $B_{y_{k}}\left(\sqrt{\delta_{k}}\right)$ with the desired precision. Similarly, one obtains that

$$
\int_{M} \delta_{k}^{\frac{n}{2}} \theta_{k}(y)^{2-n}\left|\phi_{k}(t, p)\right|(y) d v_{g}(y)=o\left(\delta_{k}^{\frac{n}{2}}\right)+o\left(\delta_{k}^{5} \mathbb{1}_{n l c f}\right) .
$$

Combining (9.2) with (7.18), (7.19), (7.20) and (7.21) one obtains (7.16).

Let us now estimate $I_{5, i}$. For $n \geq 6$, there always holds:

$$
\left|\left(u+W_{k, t, p}\right)^{2^{*}-2}-W_{k, t, p}^{2^{*}-2}\right|=O(1)
$$

Hence, for any $0 \leq i \leq n$ :

$$
\left|I_{5, i}\right| \lesssim \int_{M}\left|Z_{i, k, t, p}\right|\left|\phi_{k}(t, p)\right| d v_{g}
$$

Mimicking the computations that led to (7.19) one obtains, here also, that

$$
I_{5,0}=o\left(\delta_{k}^{\frac{n-2}{2}}\right)+o\left(\delta_{k}^{4} \mathbb{1}_{n l c f}\right)
$$

and that:

$$
I_{5, i}=o\left(\delta_{k}^{\frac{n}{2}}\right)+o\left(\delta_{k}^{5} \mathbb{1}_{n l c f}\right) .
$$

By constrast with the previous integrals, $I_{6, i}$ is easily estimated using (2.23) and (6.2). Straightforward computations give indeed that for any $0 \leq i \leq n$ :

$$
I_{6, i}=o\left(\delta_{k}^{\frac{n}{2}}\right) .
$$


Claim 7.5. There holds that:

$$
I_{7, i}=\left\{\begin{array}{l}
o\left(\delta_{k}^{\frac{n-2}{2}}\right) \text { if } i=0 \\
o\left(\delta_{k}^{\frac{n}{2}}\right) \text { if } 1 \leq i \leq n .
\end{array}\right.
$$

Proof. For any $0 \leq i \leq n$, we write:

$$
\begin{aligned}
I_{7, i} & =\int_{M}\left(\left|\mathcal{L}_{g} T+\sigma\right|_{g}^{2}+\pi^{2}\right)\left(u^{-2^{*}-1}-\left(u+W_{k, t, p}+\phi_{k}(t, p)\right)^{-2^{*}-1}\right) Z_{i, k, t, p} d v_{g} \\
& +\int_{M}\left(u+W_{k, t, p}+\phi_{k}(t, p)\right)^{-2^{*}-1}\left(\left|\mathcal{L}_{g} T+\sigma\right|_{g}^{2}-\left|\mathcal{L}_{g} T_{k, t, p}+\sigma\right|_{g}^{2}\right) Z_{i, k, t, p} d v_{g} \\
& :=I_{7, i}^{1}+I_{7, i}^{2} .
\end{aligned}
$$

We split the integral $I_{7, i}^{1}$ into an integral in $B_{y_{k}}\left(\sqrt{\delta_{k}}\right)$ and another in $M \backslash B_{y_{k}}\left(\sqrt{\delta_{k}}\right)$. Since the integrand of $I_{7, i}^{1}$ is bounded by $\left|Z_{i, k, t, p}\right|$ in $B_{y_{k}}\left(\sqrt{\delta_{k}}\right)$ (up to some positive constant that does not depend on $k$ ) and since

$$
\left|u^{-2^{*}-1}-\left(u+W_{k, t, p}+\phi_{k}(t, p)\right)^{-2^{*}-1}\right| \lesssim W_{k, t, p}+\left|\phi_{k}(t, p)\right| \text { in } M \backslash B_{y_{k}}\left(\sqrt{\delta_{k}}\right),
$$

there holds with (6.2) that:

$$
I_{7,0}^{1}=o\left(\delta_{k}^{\frac{n-2}{2}}\right)
$$

and that, for $1 \leq i \leq n$,

$$
I_{7, i}^{1}=o\left(\delta_{k}^{\frac{n}{2}}\right)
$$

To compute $I_{7, i}^{2}$, we again split the integration domain into $B_{y_{k}}\left(\sqrt{\delta_{k}}\right)$ and $M \backslash B_{y_{k}}\left(\sqrt{\delta_{k}}\right)$. On the one side, using (6.2) and (9.11), we obtain that:

$$
\begin{aligned}
\int_{B_{y_{k}}\left(\sqrt{\delta_{k}}\right)}\left(u+W_{k, t, p}+\phi_{k}(t, p)\right)^{-2^{*}-1} & \left(\left|\mathcal{L}_{g} T+\sigma\right|_{g}^{2}-\left|\mathcal{L}_{g} T_{k, t, p}+\sigma\right|_{g}^{2}\right) Z_{i, k, t, p} d v_{g} \\
& =\left\{\begin{array}{l}
o\left(\delta_{k}^{\frac{n-2}{2}}\right) \text { if } i=0 \\
o\left(\delta_{k}^{\frac{n}{2}}\right) \text { if } 1 \leq i \leq n .
\end{array}\right.
\end{aligned}
$$

On the other side, using again (9.11), we get:

$$
\begin{aligned}
\int_{M \backslash B_{y_{k}}\left(\sqrt{\delta_{k}}\right)}\left(u+W_{k, t, p}+\phi_{k}(t, p)\right)^{-2^{*}-1} & \left(\left|\mathcal{L}_{g} T+\sigma\right|_{g}^{2}-\left|\mathcal{L}_{g} T_{k, t, p}+\sigma\right|_{g}^{2}\right) Z_{i, k, t, p} d v_{g} \\
& =\left\{\begin{array}{l}
o\left(\delta_{k}^{\frac{n-2}{2}}\right) \text { if } i=0 \\
o\left(\delta_{k}^{\frac{n}{2}}\right) \text { if } 1 \leq i \leq n .
\end{array}\right.
\end{aligned}
$$

Combining (7.26)-(7.30) gives (7.25).

7.2. The $n=6$ case. In the 6 -dimensional case we need to take into account the compensation phnomenon for system (1.1), since we need to push the asymptotic estimates of the $\lambda_{k}^{i}(t, p)$ one order 
further. For this, we write the scalar equation of (7.2) as:

$$
\begin{aligned}
\sum_{i=0}^{6} \lambda_{k}^{i}(t, p) & \left(\triangle_{g}+h\right) Z_{i, k, t, p}= \\
& +\left(\triangle_{g}+h-2 f u\right) W_{k, t, p}-f\left(y_{k}\right) W_{k, t, p}^{2}+\left(f\left(y_{k}\right)-f\right) W_{k, t, p}^{2} \\
& +f \phi_{k}(t, p)^{2} \\
& +\left(\triangle_{g}+h-2 f u\right) \phi_{k}(t, p)-2 f\left(y_{k}\right) W_{k, t, p} \phi_{k}(t, p) \\
& +2\left(f\left(y_{k}\right)-f\right) W_{k, t, p} \phi_{k}(t, p) \\
& +\frac{\left|\mathcal{L}_{g} T+\sigma\right|_{g}^{2}+\pi^{2}}{u^{4}}-\frac{\left|\mathcal{L}_{g} T_{k, t, p}+\sigma\right|_{g}^{2}+\pi^{2}}{\left(u+W_{k, t, p}+\phi_{k}(t, p)\right)^{4}}
\end{aligned}
$$

and we let, for $0 \leq i \leq n$ :

$$
\begin{aligned}
& J_{1, i}=\int_{M}\left[\left(\triangle_{g}+h-2 f u\right) W_{k, t, p}-f\left(y_{k}\right) W_{k, t, p}^{2}+\left(f\left(y_{k}\right)-f\right) W_{k, t, p}^{2}\right] Z_{i, k, t, p} d v_{g}, \\
& J_{2, i}=\int_{M} f \phi_{k}(t, p)^{2} Z_{i, k, t, p} d v_{g} \\
& J_{3, i}=\int_{M}\left[\left(\triangle_{g}+h-2 f u\right) \phi_{k}(t, p)-2 f\left(y_{k}\right) W_{k, t, p} \phi_{k}(t, p)\right] Z_{i, k, t, p} d v_{g}, \\
& J_{4, i}=\int_{M} 2\left(f\left(y_{k}\right)-f\right) W_{k, t, p} \phi_{k}(t, p) Z_{i, k, t, p} d v_{g}, \\
& J_{5, i}=\int_{M}\left(\frac{\left|\mathcal{L}_{g} T+\sigma\right|_{g}^{2}+\pi^{2}}{u^{4}}-\frac{\left|\mathcal{L}_{g} T_{k, t, p}+\sigma\right|_{g}^{2}+\pi^{2}}{\left(u+W_{k, t, p}+\phi_{k}(t, p)\right)^{4}}\right) Z_{i, k, t, p} d v_{g} .
\end{aligned}
$$

These integrals are again computed in a series of Claims. The situation in the 6-dimensional case is different, since an additional contribution in the expansion of the $\lambda_{k}^{i}(t, p)$ comes from the integral $J_{5, i}$.

The computations of $J_{1, i}$ and $J_{2, i}$ follows the exact same lines than in the $n \geq 7$ case. Using (2.17), (2.21), (2.23), (2.15) and (6.2) it is esily seen that there holds:

$$
J_{1,0}=-5 K_{6}^{-6} f\left(\xi_{0}\right)^{-3} H(p) \tau_{k} \delta_{k}^{2}+o\left(\delta_{k}^{3}\right),
$$

and, for $1 \leq i \leq 6$,

$$
J_{1, i}=-30 K_{6}^{-6} f\left(\xi_{0}\right)^{-3} \nabla_{i} H(p) \frac{\tau_{k}}{\beta_{k}} \delta_{k}^{3}+o\left(\delta_{k}^{4}\right),
$$

where $K_{6}^{-6}$ is defined in (7.7). Also, mimicking the computations that led to (7.10) gives here as well:

$$
J_{2, i}=\left\{\begin{array}{l}
o\left(\delta_{k}^{3}\right), \text { if } i=0, \\
o\left(\delta_{k}^{4}\right), \text { if } 1 \leq i \leq 6 .
\end{array}\right.
$$

Claim 7.6. There holds:

$$
J_{3,0}=o\left(\delta_{k}^{3}\right)
$$

and, for any $1 \leq i \leq 6$ :

$$
J_{3, i}=o\left(\delta_{k}^{4}\right)
$$


Proof. As for the $n \geq 7$ case, we rewrite $J_{3, i}$ as:

$$
J_{3, i}=\int_{M}\left(\left[\triangle_{g}+(h-2 f u)\right] Z_{i, k, t, p}-2 f\left(y_{k}\right) W_{k, t, p} Z_{i, k, t, p}\right) \phi_{k}(t, p) d v_{g} .
$$

Compared to the $n \geq 7$ case, the estimation of $J_{3, i}$ requires the additional information on $\phi_{k}(t, p)$ given by Proposition 6.3. Namely, using (6.24) for a suitable choice of a sequence $\left(R_{k}\right)_{k}$ yields:

$$
\left.\int_{M}\left(\delta_{k}^{2} r_{k}^{-6} \mathbb{1}_{r_{k} \leq d_{g_{y_{k}}} \leq 2 r_{k}}+\delta_{k}^{2} \mathbb{1}_{n l c f}\right)\right) \phi_{k}(t, p) d v_{g}=o\left(\delta_{k}^{3}\right)
$$

while using (2.7) and (6.2) gives:

$$
\int_{M}\left(h-\frac{1}{5} S_{g}-2 f u\right) Z_{0, k, t, p} \phi_{k}(t, p) d v_{g}=o\left(\delta_{k}^{3}\right) .
$$

If $(M, g)$ is not locally conformally flat there holds, for some $R_{k}$ satisfying $R_{k} \sqrt{\delta_{k}}=o(1)$ :

$$
\begin{aligned}
\int_{M} & \frac{1}{5} \Lambda_{y_{k}} S_{g_{y_{k}}} Z_{0, k, t, p} \phi_{k}(t, p) d v_{g} \\
& =\int_{M \backslash B_{y_{k}}\left(R_{k} \sqrt{\delta_{k}}\right)} \frac{1}{5} \Lambda_{y_{k}} S_{g_{y_{k}}} Z_{0, k, t, p} \phi_{k}(t, p) d v_{g}+\int_{B_{y_{k}}\left(R_{k} \sqrt{\delta_{k}}\right) \backslash B_{y_{k}}\left(\sqrt{\delta_{k}}\right)} \frac{1}{5} \Lambda_{y_{k}} S_{g_{y_{k}}} Z_{0, k, t, p} \phi_{k}(t, p) d v_{g} \\
& +\int_{B_{y_{k}}\left(\sqrt{\delta_{k}}\right)} \frac{1}{5} \Lambda_{y_{k}} S_{g_{y_{k}}} Z_{0, k, t, p} \phi_{k}(t, p) d v_{g} \\
& =o\left(\delta_{k}^{3}\right),
\end{aligned}
$$

where we used again (2.4) and we estimated the first integral using (6.24), the second one using (6.2) and the third one using Proposition 6.2. With (9.2) we therefore obtain (7.34).

Let now $1 \leq i \leq 6$. Similarly, using (6.24) for a suitable radius $R_{k}$ and using (2.15) yields:

$$
\int_{M} \delta_{k}^{3} r_{k}^{-7}\left|\phi_{k}(t, \xi)\right| d v_{g}=o\left(\delta_{k}^{4}\right)
$$

and as in (7.36) (6.24), (6.2), Proposition 6.2 and (2.4) show that if $(M, g)$ is not locally conformally flat, then:

Independently, there holds that:

$$
\int_{M} \frac{1}{5} \Lambda_{y_{k}} S_{g_{y_{k}}} Z_{i, k, t, p} \phi_{k}(t, p) d v_{g}=o\left(\delta_{k}^{4}\right)
$$

$$
\begin{aligned}
& \int_{M}\left(h-\frac{1}{5} S_{g}-2 f u\right) Z_{i, k, t, p} \phi_{k}(t, p) d v_{g} \\
& =\int_{B_{y_{k}}\left(\sqrt{\delta_{k}}\right)}\left(h-\frac{1}{5} S_{g}-2 f u\right) Z_{i, k, t, p} \phi_{k}(t, p) d v_{g} \\
& \quad+\int_{M \backslash B_{y_{k}}\left(\sqrt{\delta_{k}}\right)}\left(h-\frac{1}{5} S_{g}-2 f u\right) Z_{i, k, t, p} \phi_{k}(t, p) d v_{g} \\
& =o\left(\delta_{k}^{4}\right)
\end{aligned}
$$

where we used (2.7) and Proposition 6.2 to estimate the first integral and (2.7) and (6.2) to estimate the second one. Finally, in case $(M, g)$ is not locally conformally flat, mimicking the computations that led to (7.36) we get that:

$$
\int_{M} \delta_{k}^{3} \theta_{k}(\cdot)^{-4}\left|\phi_{k}(t, p)\right| d v_{g}=o\left(\delta_{k}^{4}\right) .
$$

Combining the above estimates with (9.2) gives in the end (7.35). 
Here again, $J_{4, i}$ is easily estimated : straightforward computations using (2.23) give indeed that, for any $0 \leq i \leq 6$ :

$$
J_{4, i}=o\left(\delta_{k}^{4}\right) .
$$

As already mentioned, and unlike in the higher-dimensional case, the coupling field $X$ enters the expansion of $J_{5, i}$ for $n=6$ :

Claim 7.7. There holds:

$$
J_{5,0}=\kappa \delta_{k}^{3}+o\left(\delta_{k}^{3}\right)
$$

where the constant $\kappa$ is explicitly given by (7.45) below, and, for any $1 \leq i \leq 6$ :

$$
J_{5, i}=O\left(\delta_{k}^{\frac{7}{2}}\right) \text {. }
$$

Proof. We again write that there holds, for any $0 \leq i \leq 6$ :

$$
\begin{aligned}
J_{5, i} & =\int_{M}\left(\left|\mathcal{L}_{g} T+\sigma\right|_{g}^{2}+\pi^{2}\right)\left(u^{-4}-\left(u+W_{k, t, p}+\phi_{k}(t, p)\right)^{-4}\right) Z_{i, k, t, p} d v_{g} \\
& +\int_{M}\left(u+W_{k, t, p}+\phi_{k}(t, p)\right)^{-4}\left(\left|\mathcal{L}_{g} T+\sigma\right|_{g}^{2}-\left|\mathcal{L}_{g} T_{k, t, p}+\sigma\right|_{g}^{2}\right) Z_{i, k, t, p} d v_{g} \\
& :=J_{5, i}^{1}+J_{5, i}^{2} .
\end{aligned}
$$

Let $R>0$. Since there holds

$$
\left|u^{-4}-\left(u+W_{k, t, p}+\phi_{k}(t, p)\right)^{-4}\right| \lesssim\left(W_{k, t, p}+\left|\phi_{k}(t, p)\right|\right) \text { in } M \backslash B_{y_{k}}\left(R \sqrt{\delta_{k}}\right),
$$

then (6.24) shows that:

$$
\left|\int_{M \backslash B_{y_{k}}\left(R \sqrt{\delta_{k}}\right)}\left(\left|\mathcal{L}_{g} T+\sigma\right|_{g}^{2}+\pi^{2}\right)\left(u^{-4}-\left(u+W_{k, t, p}+\phi_{k}(t, p)\right)^{-4}\right) Z_{0, k, t, p} d v_{g}\right| \lesssim \frac{\delta_{k}^{3}}{R^{2}}+o\left(\delta_{k}^{3}\right) .
$$

Independently, by (6.2), Lebesgue's dominated convergence theorem shows that there holds:

$$
\begin{aligned}
\delta_{k}^{-3} & \int_{B_{y_{k}}\left(R \sqrt{\delta_{k}}\right)}\left(\left|\mathcal{L}_{g} T+\sigma\right|_{g}^{2}+\pi^{2}\right)\left(u^{-4}-\left(u+W_{k, t, p}+\phi_{k}(t, p)\right)^{-4}\right) Z_{0, k, t, p} d v_{g} \\
\quad= & \frac{(24)^{2}}{f\left(\xi_{0}\right)}\left(\left|\mathcal{L}_{g} T+\sigma\right|_{g}^{2}+\pi^{2}\right)\left(\xi_{0}\right) \int_{B_{0}(R)}\left[u\left(\xi_{0}\right)^{-4}-\left(u\left(\xi_{0}\right)+\left(\frac{24}{f\left(\xi_{0}\right)}\right)^{2}|y|^{-4}\right)^{4}\right]|y|^{-4} d y+o(1),
\end{aligned}
$$

as $k \rightarrow+\infty$, so that (7.41) and (7.42) together show that there holds

$$
\begin{aligned}
J_{5,0}^{1} & =\frac{(24)^{2}}{f\left(\xi_{0}\right)}\left(\left|\mathcal{L}_{g} T+\sigma\right|_{g}^{2}+\pi^{2}\right)\left(\xi_{0}\right) \\
& \times \int_{\mathbb{R}^{6}}\left[u\left(\xi_{0}\right)^{-4}-\left(u\left(\xi_{0}\right)+\left(\frac{24}{f\left(\xi_{0}\right)}\right)^{2}|y|^{-4}\right)^{4}\right]|y|^{-4} d y \cdot \delta_{k}^{3}+o\left(\delta_{k}^{3}\right) .
\end{aligned}
$$

Using (9.10) with (6.3) one gets that:

$$
\left|\mathcal{L}_{g} T+\sigma\right|_{g}^{2}-\left|\mathcal{L}_{g} T_{k, t, \xi}+\sigma\right|_{g}^{2}=-\left|\mathcal{L}_{g} \Theta_{k}\right|_{g}^{2}+O\left(\left|\mathcal{L}_{g} \Theta_{k}\right|_{g}\right)+O\left(\delta_{k}\right),
$$

where $\Theta_{k}$ is defined in (9.9) below. Using the asymptotic (9.17) together with (2.24) and the dominated convergence theorem show that:

$$
\begin{aligned}
& J_{5,0}^{2}=-C(6) f\left(\xi_{0}\right)^{-8} \alpha^{2} \\
& \times \int_{\mathbb{R}^{6}}\left(u\left(\xi_{0}\right)+\left(\frac{f\left(\xi_{0}\right)}{24}\right)^{-2}|y|^{-4}\right)^{-4}\left(2+28\left|\left\langle\frac{Z(0)}{|Z(0)|_{\text {eucl }}}, \frac{y}{|y|}\right\rangle\right|^{2}\right)|y|^{-14} d y \cdot \delta_{k}^{3},
\end{aligned}
$$


where we have let $C(6)=2^{32} 3^{9} 5^{-4}$ and where $\alpha$ is as in (2.25). Combining (7.43) and (7.44) in (7.40) one obtains in the end (7.38), where $\kappa$ is given by:

$$
\begin{aligned}
\kappa & =\frac{(24)^{2}}{f\left(\xi_{0}\right)}\left(\left|\mathcal{L}_{g} T+\sigma\right|_{g}^{2}+\pi^{2}\right)\left(\xi_{0}\right) \times \int_{\mathbb{R}^{6}}\left[u\left(\xi_{0}\right)^{-4}-\left(u\left(\xi_{0}\right)+\left(\frac{24}{f\left(\xi_{0}\right)}\right)^{2}|y|^{-4}\right)^{4}\right]|y|^{-4} d y \\
& -C(6) f\left(\xi_{0}\right)^{-8} \alpha^{2} \times \int_{\mathbb{R}^{6}}\left(u\left(\xi_{0}\right)+\left(\frac{f\left(\xi_{0}\right)}{24}\right)^{-2}|y|^{-4}\right)^{-4}\left(2+28\left|\left\langle\frac{Z(0)}{|Z(0)|_{\text {eucl }}}, \frac{y}{|y|}\right\rangle\right|^{2}\right)|y|^{-14} d y .
\end{aligned}
$$

In particular, up to choosing $\alpha$ as in (2.25) small enough, we have $\kappa>0$.

Let now $1 \leq i \leq 6$. Mimicking the proof of (7.41) and (7.42) gives, by (6.24) and the dominated convergence theorem, that

$$
J_{5, i}^{1}=o\left(\delta_{k}^{\frac{7}{2}}\right)
$$

Finally, using the expansion (9.10) along with (9.17) shows, again by dominated convergence, that there holds:

With (7.40) we obtain that (77.39) holds.

$$
J_{5, i}^{2}=O\left(\delta_{k}^{\frac{7}{2}}\right)
$$

\section{Conclusive argument}

In this section we conclude the proof of Theorem 1.1. We use the expansions of the $\lambda_{k}^{i}(t, p)$ obtained in Section 7 to show that, for any $k$, there exist $\left(t_{k}, p_{k}\right) \in[1 / D, D] \times \overline{B_{0}(1)}$ such that $\lambda_{k}^{i}\left(t_{k}, p_{k}\right)=0$ for any $0 \leq i \leq n$. We use here the notations of Section 7 . In particular, $\delta_{k}$ and $y_{k}$ are defined by (2.16) and (7.1).

First, for any $0 \leq i \leq n$, there holds:

$$
\int_{M}\left\langle\nabla Z_{i, k, t, p}, \nabla Z_{j, k, t, p}\right\rangle_{g}+h Z_{i, k, t, p} Z_{j, k, t, p} d v_{g}=\delta_{i j}\left\|\nabla V_{i, y_{k}}\right\|_{L^{2}\left(\mathbb{R}^{n}\right)}^{2}+O\left(\delta_{k}\right),
$$

uniformly in the choice of $h, t$ and $p$, and where $V_{i, y_{k}}$ is defined in (2.18).

Assume first that $(M, g)$ is locally conformally flat or that $7 \leq n \leq 10$. Then, combining (7.5), (7.6), (7.8), (7.9), (7.10), (7.13), (7.14), (77.15), (7.16), (7.22), (7.23), (7.24) and (7.25) in (7.4) yields, with (8.1) and (2.6), that:

$$
\begin{aligned}
& \left(I_{n+1}+O\left(\delta_{k}\right)\right)\left(\begin{array}{c}
\lambda_{k}^{0}(t, p) \\
\vdots \\
\lambda_{k}^{n}(t, p)
\end{array}\right) \\
& =\left(\begin{array}{r}
\mu_{k}^{\frac{n-2}{2}}\left[\frac{8(n-1)}{(n-2)(n-4)} K_{n}^{-n} f\left(\xi_{0}\right)^{-\frac{n}{2}} H(p) t^{2}-\frac{1}{2}(n-2)^{2}(n(n-2))^{\frac{n-2}{2}} \omega_{n-1} f\left(\xi_{0}\right)^{1-\frac{n}{2}} u\left(\xi_{0}\right) t^{\frac{n-2}{2}}\right. \\
\left.-\frac{10}{9} f\left(\xi_{0}\right)^{-4} K_{10}^{-10}\left|W_{g}(\xi)\right|_{g}^{2} t^{4} \mathbb{1}_{n=10}+R_{k}^{0}(t, p)\right] \\
\frac{\mu_{k}^{\frac{n}{2}}}{\beta_{k}}\left[\frac{2 n(n-1)}{n-4} K_{n}^{-n} f\left(\xi_{0}\right)^{-\frac{n}{2}} \nabla_{i} H(p) t^{3}+R_{k}^{i}(t, p)\right]
\end{array}\right),
\end{aligned}
$$

where, for $0 \leq i \leq n, R_{k}^{i}(t, p)$ denotes a function which converges to zero in $C^{0}\left([1 / D, D] \times \overline{B_{0}(1)}\right)$ as $k \rightarrow+\infty$, where $K_{n}^{-n}$ is as in (17.7) and where $\beta_{k}$ is as in (2.6). The continuity of $R_{k}^{i}, 0 \leq i \leq n$, 
is a direct consequence of the continuity of $\phi_{k}$ as stated in Proposition 6.1. Let $F$ be the function defined in $[1 / D, D] \times \overline{B_{0}(1)}$ by:

$$
F(t, p)=\left(\begin{array}{rr}
\frac{8(n-1)}{(n-2)(n-4)} K_{n}^{-n} f\left(\xi_{0}\right)^{-\frac{n}{2}} H(p) t^{2}-\frac{1}{2}(n-2)^{2}(n(n-2))^{\frac{n-2}{2}} \omega_{n-1} f\left(\xi_{0}\right)^{1-\frac{n}{2}} u\left(\xi_{0}\right) t^{\frac{n-2}{2}} \\
\frac{2 n(n-1)}{n-4} K_{n}^{-n} f\left(\xi_{0}\right)^{-\frac{n}{2}} \nabla_{i} H(p) t^{3} & -\frac{10}{9} f\left(\xi_{0}\right)^{-4} K_{10}^{-10}\left|W_{g}(\xi)\right|_{g}^{2} t^{4} \mathbb{1}_{n=10}
\end{array}\right)
$$

and let $t_{0}>0$ be the unique solution of:

$$
\begin{aligned}
\frac{8(n-1)}{(n-2)(n-4)} K_{n}^{-n} f\left(\xi_{0}\right)^{-\frac{n}{2}} t^{2}=\frac{1}{2}(n-2)^{2}( & n(n-2))^{\frac{n-2}{2}} \omega_{n-1} f\left(\xi_{0}\right)^{1-\frac{n}{2}} u\left(\xi_{0}\right) t^{\frac{n-2}{2}} \\
& +\frac{10}{9} f\left(\xi_{0}\right)^{-4} K_{10}^{-10}\left|W_{g}(\xi)\right|_{g}^{2} t^{4} \mathbb{1}_{n=10} .
\end{aligned}
$$

Using the assumption that 0 is a non-degenerate critical point of $H$, it is easily checked that the differential of $F$ at $\left(t_{0}, 0\right)$ is invertible. Since there holds $F\left(t_{0}, 0\right)=0$ by definition of $t_{0}$, and since the $R_{k}^{i}(t, p)$ appearing in (8.2) uniformly converge to 0 as $k \rightarrow+\infty$, standard degree-theoretic arguments yield the existence of a sequence $\left.\left(t_{k}, p_{k}\right) \in\right] 1 / D, D\left[\times B_{0}(1)\right.$ of interior points such that

$$
F\left(t_{k}, p_{k}\right)+\left(\begin{array}{l}
R_{k}^{0}\left(t_{k}, p_{k}\right) \\
\left(R_{k}^{i}\left(t_{k}, p_{k}\right)\right)_{1 \leq i \leq n}
\end{array}\right)=0
$$

for any $k$. Note that throughout this argument we assumed that $D$ is chosen large enough to have $t_{0} \in[2 / D, D / 2]$. Coming back to (8.2), this amounts to say that $\lambda_{k}^{i}\left(t_{k}, p_{k}\right)=0$ for any $0 \leq i \leq n$. And with Proposition 6.1 and (7.2) this shows that the function $u_{k, t_{k}, p_{k}}$ is a solution of system (1.1), and concludes the proof of Theorem 1.1 in this case.

If now we assume that $n \geq 11$ and $(M, g)$ is not locally conformally flat, the same arguments lead to the following expansion for the $\lambda_{k}^{i}(t, p)$ :

$$
\begin{aligned}
& \left(I_{n+1}+O\left(\delta_{k}\right)\right)\left(\begin{array}{c}
\lambda_{k}^{0}(t, p) \\
\vdots \\
\lambda_{k}^{n}(t, p)
\end{array}\right) \\
& =K_{n}^{-n} f\left(\xi_{0}\right)^{-\frac{n}{2}}\left(\begin{array}{c}
\mu_{k}^{4}\left[\frac{8(n-1)}{(n-2)(n-4)} H(p) t^{2}-\frac{1}{3} \frac{n(n-2)}{(n-4)(n-6)} f\left(\xi_{0}\right)\left|W_{g}\left(\xi_{0}\right)\right|_{g}^{2} t^{4}+R_{k}^{0}(t, p)\right] \\
\frac{\mu_{k}^{5}}{\beta_{k}}\left[\frac{2 n(n-1)}{n-4} \nabla_{i} H(p) t^{3}+R_{k}^{i}(t, p)\right]
\end{array}\right) .
\end{aligned}
$$

While in the 6-dimensional case, we end up with:

$$
\left(I_{7}+O\left(\delta_{k}\right)\right)\left(\begin{array}{c}
\lambda_{k}^{0}(t, p) \\
\vdots \\
\lambda_{k}^{6}(t, p)
\end{array}\right)=\left(\begin{array}{c}
\mu_{k}^{3}\left[-5 K_{6}^{-6} f\left(\xi_{0}\right)^{-3} H(p) t^{2}+\kappa t^{3}+R_{k}^{0}(t, p)\right] \\
\frac{\mu_{k}^{4}}{\beta_{k}}\left[-30 K_{6}^{-6} f\left(\xi_{0}\right)^{-3} \nabla_{i} H(p) t^{3}+R_{k}^{i}(t, p)\right]
\end{array}\right)
$$

where the constant $\kappa$ is defined in (7.45). The conclusion in these cases follows then from the exact same arguments than in the previous case, thus concluding the proof of Theorem 1.1 


\section{TeChNiCAl RESUlts}

9.1. Conformal laplacian of $W_{k, t, \xi}$ and of the $Z_{i, k, t, \xi}, 0 \leq i \leq n$. Below are given the expressions of the conformal laplacian of the functions $W_{k}$ and $Z_{i, k, t, \xi}$ defined in (2.17) and (2.21). Let $D>0$ and let $\left(t_{k}, \xi_{k}\right)_{k}$ be a sequence of points in $[1 / D, D] \times M$. Then, for any function $h \in C^{\infty}(M)$, there holds:

$$
\begin{array}{r}
\left(\triangle_{g}+h\right) W_{k, t_{k}, \xi_{k}}=f\left(\xi_{k}\right) W_{k, t_{k}, \xi_{k}}^{2^{*}-1}+\left(h-c_{n} S_{g}\right) W_{k, t_{k}, \xi_{k}}+c_{n} \Lambda_{\xi_{k}}^{2^{*}-2} S_{g_{\xi_{k}}} W_{k, t_{k}, \xi_{k}} \\
+O\left(\delta_{k}^{\frac{n-2}{2}} \mathbb{1}_{d_{k} \leq 2 r_{k}}\right)+O\left(\delta_{k}^{\frac{n-2}{2}} r_{k}^{-n^{n}} \mathbb{1}_{r_{k} \leq d_{k} \leq 2 r_{k}}\right),
\end{array}
$$

and, for any $1 \leq i \leq n$ :

$$
\begin{gathered}
\left(\triangle_{g}+h\right) Z_{0, k, t_{k}, \xi_{k}}=\left(2^{*}-1\right) f\left(\xi_{k}\right) W_{k, t_{k}, \xi_{k}}^{2^{*}-2} Z_{0, k, t_{k}, \xi_{k}}+\left(h-c_{n} S_{g}\right) Z_{0, k, t_{k}, \xi_{k}} \\
\quad+c_{n} \Lambda_{\xi_{k}}^{2^{*}-2} S_{g_{\xi}} Z_{0, k, t_{k}, \xi_{k}}+O\left(\delta_{k}^{\frac{n-2}{2}} r_{k}^{-n} \mathbb{1}_{r_{k} \leq d_{g_{\xi_{k}}} \leq 2 r_{k}}\right)+O\left(\delta_{k}^{\frac{n-2}{2}} \mathbb{1}_{n l c f, d_{g_{\xi_{k}}} \leq 2 r_{k}}\right), \\
\left(\triangle_{g}+h\right) Z_{i, k, t_{k}, \xi_{k}}=\left(2^{*}-1\right) f\left(\xi_{k}\right) W_{k, t_{k}, \xi_{k}}^{2^{*}-2} Z_{i, k, t_{k}, \xi_{k}}+\left(h-c_{n} S_{g}\right) Z_{i, k, t_{k}, \xi_{k}} \\
\quad+c_{n} \Lambda_{\xi_{k}}^{2^{*}-2} S_{g_{\xi_{k}}} Z_{i, k, t_{k}, \xi_{k}}+O\left(\delta_{k}^{\frac{n}{2}} r_{k}^{-n-1} \mathbb{1}_{r_{k} \leq d_{g_{\xi_{k}}} \leq 2 r_{k}}\right)+O\left(\delta_{k}^{\frac{n}{2}} \theta_{k}(\cdot)^{2-n_{1}} \mathbb{1}_{n l c f, d_{g_{\xi_{k}}} \leq 2 r_{k}}\right) .
\end{gathered}
$$

In (9.1) and (9.2) $\Lambda_{\xi_{k}}$ is as in (2.3) and $d_{k}=d_{g_{\xi_{k}}}\left(\xi_{k}, \cdot\right)$. The notation " $O(f)^{\prime \prime}$ denotes a smooth function which can be uniformly bounded in $C^{0}(M)$ by $C_{0}|f|$, where $C_{0}$ is some positive constant that does not depend on $k$. Also, the notational shorthand $\mathbb{1}_{n l c f}$ is used to indicate that the corresponding term vanishes if $(M, g)$ is locally conformally flat in a fixed neighbourhood of $\xi_{k}$. The notations $\mathbb{1}_{r_{k} \leq d_{g_{\xi_{k}}}} \leq 2 r_{k}$ and $\mathbb{1}_{n l c f, d_{g_{\xi_{k}}} \leq 2 r_{k}}$ are defined similarly.

These expressions are obtained from (2.17) and (2.21), using the conformal invariance property of the conformal laplacian and the properties of the normal conformal factor $\Lambda_{\xi_{k}}$. See for instance Esposito-Pistoia-Vétois [23] and Lee-Parker [32] for more details.

9.2. Pointwise estimates for solutions of the 1-form equation. Let $(M, g)$ be a closed Riemannian manifold possessing no conformal Killing fields. This means that the operator $\vec{\triangle}_{g}$, when acting on $H^{1}$ fields of 1-forms, has zero kernel. We start by recalling that the operator $\vec{\triangle}_{g}$ always possesses local Green fields:

Proposition 9.1. Let $x_{0} \in M$ and $\delta<\frac{i_{g}(M)}{2}$. For any $x \in B_{x_{0}}(\delta)$ there exist $n$ fields of 1-forms $G_{1}(x, \cdot), \cdots, G_{n}(x, \cdot)$ defined in $B_{x_{0}}(\delta) \backslash\{x\}$ which form a fundamental solution for the operator $\vec{\triangle}_{g}$ in $B_{x_{0}}(\delta)$ in the following sense: for any 1 -form $X \in \Gamma\left(T B_{x_{0}}(\delta)\right)$ with $X \equiv 0$ on $\partial B_{x_{0}}(\delta)$, there holds:

$$
(X-\pi(X))_{i}(x)=\int_{B_{x_{0}}(\delta)}\left\langle G_{i}(x, y), \vec{\triangle}_{g} X(y)\right\rangle_{g(y)} d v_{g}(y),
$$

where $\pi$ denotes the orthogonal projection for the $L^{2}\left(B_{x_{0}}(\delta)\right)$-scalar product on the set

$$
\left\{X \in H^{1}\left(B_{x_{0}}(\delta)\right), \mathcal{L}_{g} X=0 \text { in } B_{x_{0}}(\delta)\right\},
$$

and where the coordinates in (9.3) are taken in any chart in $B_{x_{0}}(\delta)$. The Green fields satisfy in addition: for any $1 \leq i \leq n$, for any $x \in B_{x_{0}}(\delta / 2), y \in B_{x_{0}}(\delta)$,

$$
d_{g}(x, y)\left|\nabla G_{i}(x, y)\right|_{g}+\left|G_{i}(x, y)\right|_{g} \leq C(n, g) d_{g}(x, y)^{2-n},
$$

where the derivative in (9.4) can be taken with respect to $x$ or to $y$.

The construction of these Green fields is carried out in the Euclidean case in Druet-Premoselli [21] in dimension 3 and in Premoselli [39]. The extension to the Riemannian case shows no additional 
difficulty. The proof just consists in adapting the steps that lead to the construction of the Riemannian Green function for the Laplace-Beltrami operator on a Riemannian manifold starting from the euclidean one (see for instance Appendix A in Druet-Hebey-Robert [18 or Robert [43]), and we will not detail it here. Using the Euclidean expression computed in Premoselli [39] one finds that these Green fields $G_{i}$ have the following expansion:

$$
G_{i}\left(x, \exp _{x}(y)\right)_{j}=-\frac{1}{4(n-1) \omega_{n-1}}|y|^{2-n}\left((3 n-2) \delta_{i j}+(n-2) \frac{y_{i} y_{j}}{|y|^{2}}\right)(1+O(|y|)),
$$

for any $x \in M$, where the exponential map is taken here for the background metric $g$ and $\omega_{n-1}$ is the volume of the standard $(n-1)$-sphere in $\mathbb{R}^{n}$. If $X$ is a smooth 1 -form in $B_{x_{0}}(\delta)$ which vanishes on $\partial B_{x_{0}}(\delta)$ formula (9.3) can be differentiated to obtain: for any $1 \leq i, j \leq n$, for any $x \in B_{x_{0}}(\delta)$,

$$
\mathcal{L}_{g} X_{i j}(x)=\int_{B_{x_{0}}(\delta)}\left\langle H_{i j}(x, y), \vec{\triangle}_{g} X(y)\right\rangle_{g(y)} d v_{g}(y),
$$

where we have let:

$$
H_{i j}(x)=\nabla_{i} G_{j}(x, y)+\nabla_{j} G_{i}(x, y)-\frac{2}{n} g^{k l}(x) \nabla_{k} G_{l}(x, y) g_{i j}(x),
$$

and the covariant derivatives are all taken here with respect to $x$.

We now use these Green fields to derive optimal pointwise estimates on solutions of the 1-form equation. Let $u$ be a smooth positive function in $M$. Let $D>0$ and let $\left(t_{k}, \xi_{k}\right)_{k} \in\left[\frac{1}{D}, D\right] \times M$ be a sequence of points, and consider the function $W_{k, t_{k}, \xi_{k}}$ given by (2.17), where $\delta_{k}$ is given by (2.16) and $\left(\mu_{k}\right)_{k}$ denotes some sequence of positive numbers which converge to zero. Recall that the functions $W_{k, t_{k}, \xi_{k}}$ - that shall now be abbreviated as $W_{k}$ - are compactly supported on a ball centered at $\xi_{k}$ and of radius $2 r_{k}$ given by (2.15) (the ball is taken here with respect to the metric $\left.g_{\xi_{k}}\right)$. Let $\left(\varepsilon_{k}\right)_{k}$ denote a sequence of positive numbers and, for any $k$, let $v_{k}$ be a continuous function satisfying

$$
\left\|\frac{v}{u+W_{k}}\right\|_{C^{0}(M)} \leq \varepsilon_{k} .
$$

Let $X$ and $Y$ be two 1-forms in $M$ and, for any $k$, let $T_{k}, \Theta_{k}$ and $T$ be the unique solutions of the following equations in $M$ :

$$
\begin{aligned}
& \vec{\triangle}_{g} T_{k}=\left(u+W_{k}+v_{k}\right)^{2^{*}} X+Y, \\
& \vec{\triangle}_{g} \Theta_{k}=W_{k}^{2^{*}} X, \\
& \vec{\triangle}_{g} T=u^{2^{*}} X+Y .
\end{aligned}
$$

Then the following pointwise asymptotic estimate on $\mathcal{L}_{g} T_{k}$ holds:

Proposition 9.2. For any sequence $\left(x_{k}\right)_{k}$ of points in $M$, there holds:

$$
\mathcal{L}_{g} T_{k}\left(x_{k}\right)=\left(1+O\left(\delta_{k}\right)\right) \mathcal{L}_{g} \Theta_{k}\left(x_{k}\right)+\mathcal{L}_{g} T\left(x_{k}\right)+O\left(\|X\|_{C^{0}(M)} \varepsilon_{k}+\left|X\left(\xi_{k}\right)\right|_{g}+\delta_{k}\|\nabla X\|_{L^{\infty}\left(2 r_{k}\right)}\right),
$$

uniformly in $k$. As a consequence, there holds, for any $x \in M$ :

$$
\left|\mathcal{L}_{g} T_{k}-\mathcal{L}_{g} T\right|_{g}(x) \leq C\left(\left[\left|X\left(\xi_{k}\right)\right|_{g}+\delta_{k}\|\nabla X\|_{L^{\infty}\left(2 r_{k}\right)}\right] \theta_{k}(x)^{1-n}+\|X\|_{C^{0}(M)} \varepsilon_{k}\right),
$$

where $C$ is a positive constant independent of $k$ and $\|X\|_{C^{0}(M)}$ and where $\theta_{k}(x)$ is as in (4.25). 
Proof. Let $G_{i}, 1 \leq i \leq n$, be the family of Green fields given by Proposition 9.1 and defined in $B_{\xi_{k}}(\delta) \times B_{\xi_{k}}(\delta) \backslash\{x=y\}$. Let $\eta \in C_{c}^{\infty}\left(\mathbb{R}^{+}\right)$be a smooth cut-off function equal to 1 in $[0, \delta / 4]$ and equal to 0 outside of $[0, \delta / 2]$. We define the following 1 -forms, compactly supported in $B_{\xi_{k}}(\delta / 2)$ :

$$
P_{k}(x)_{i}=\left(\int_{B_{x_{0}}\left(\frac{\delta}{2}\right)}\left\langle G_{i}(x, y),\left[\left(u+W_{k}+v_{k}\right)^{2^{*}}-u^{2^{*}}\right](y) X(y)\right\rangle_{g(y)} d v_{g}(y)\right) \cdot \eta\left(d_{g}\left(\xi_{k}, x\right)\right) .
$$

By definition of $G_{i}$, by the choice of $\eta$, by (9.4), (9.8) and by Giraud's lemma there holds, for any $x \in B_{\xi_{k}}(\delta / 2)$ :

$$
\begin{aligned}
& \left|P_{k}\right|(x) \leq C\left(\left|X\left(\xi_{k}\right)\right|_{g}+\delta_{k}\|\nabla X\|_{L^{\infty}\left(2 r_{k}\right)}\right) \theta_{k}(x)^{2-n}+C\|X\|_{C^{0}(M)} \varepsilon_{k} \\
& \left|\mathcal{L}_{g} P_{k}\right|_{g}(x) \leq C\left(\left|X\left(\xi_{k}\right)\right|_{g}+\delta_{k}\|\nabla X\|_{L^{\infty}\left(2 r_{k}\right)}\right) \theta_{k}(x)^{1-n}+C\|X\|_{C^{0}(M)} \varepsilon_{k} .
\end{aligned}
$$

In (9.13) and until the end of this section $C$ will denote some positive constant that does not depend on $k$ or on $\|X\|_{C^{0}(M)}$. Since $\left|\left(u+W_{k}+v_{k}\right)^{2^{*}}-u^{2^{*}}\right| \leq C \varepsilon_{k}$ in $M \backslash B_{\xi_{k}}(\delta / 4)$, and by definition of $G_{i}$, one obtains with (9.9), (9.12) and (9.13) that:

$$
\begin{aligned}
& \vec{\triangle}_{g}\left(T_{k}-P_{k}-T\right)=0 \text { in } B_{\xi_{k}}(\delta / 4) \\
&\left|\vec{\triangle}_{g}\left(T_{k}-P_{k}-T\right)\right| \leq C\left(\left|X\left(\xi_{k}\right)\right|_{g}+\delta_{k}\|\nabla X\|_{L^{\infty}\left(2 r_{k}\right)}+\|X\|_{C^{0}(M)} \varepsilon_{k}\right) \text { in } B_{\xi_{k}}(\delta / 2) \backslash B_{\xi_{k}}(\delta / 4) \\
&\left|\vec{\triangle}_{g}\left(T_{k}-P_{k}-T\right)\right| \leq C\|X\|_{C^{0}(M)} \varepsilon_{k} \text { in } M \backslash B_{\xi_{k}}(\delta / 2) .
\end{aligned}
$$

Standard elliptic regularity theory applies for $\vec{\triangle}_{g}$ (see e.g. Premoselli [39]), and with the latter estimates shows that there holds, in $C^{0}(M)$ :

$$
\mathcal{L}_{g} T_{k}=\mathcal{L}_{g} P_{k}+\mathcal{L}_{g} T+O\left(\left|X\left(\xi_{k}\right)\right|_{g}+\delta_{k}\|\nabla X\|_{L^{\infty}\left(2 r_{k}\right)}+\|X\|_{C^{0}(M)} \varepsilon_{k}\right) .
$$

Independently, similar arguments show that there holds:

$$
\mathcal{L}_{g} \Theta_{k}=\mathcal{L}_{g} Q_{k}+O\left(\left|X\left(\xi_{k}\right)\right|_{g}+\delta_{k}\|\nabla X\|_{L^{\infty}\left(2 r_{k}\right)}\right),
$$

where $\Theta_{k}$ is defined in (9.9) and where we have let, for $1 \leq i \leq n$ :

$$
Q_{k}(x)_{i}=\left(\int_{B_{x_{0}}\left(\frac{\delta}{2}\right)}\left\langle G_{i}(x, y), W_{k}^{2^{*}}(y) X(y)\right\rangle_{g(y)} d v_{g}(y)\right) \cdot \eta\left(d_{g}\left(\xi_{k}, x\right)\right) .
$$

Since there holds, in $B_{\xi_{k}}(\delta / 4)$, that

$$
\left|\left(u+W_{k}+v_{k}\right)^{2^{*}}-u^{2^{*}}-W_{k}^{2^{*}}\right| \leq C\left(\varepsilon_{k}+W_{k}^{2^{*}-1}+W_{k}\right),
$$

we obtain with Giraud's lemma, (9.12), (9.15) and (9.16) that, for any sequence $\left(x_{k}\right)_{k}$ in $M$ :

$$
\mathcal{L}_{g} P_{k}\left(x_{k}\right)=\left(1+O\left(\delta_{k}\right)\right) \mathcal{L}_{g} \Theta_{k}\left(x_{k}\right)+O\left(\left|X\left(\xi_{k}\right)\right|_{g}+\delta_{k}\|\nabla X\|_{L^{\infty}\left(2 r_{k}\right)}+\|X\|_{C^{0}(M)} \varepsilon_{k}\right) .
$$

Then estimate (9.10) then follows from the latter expansion and (9.14), and (9.11) follows from (9.10). 
Using (9.5), (9.6), (9.7), (9.15) and (9.16) one obtains the following asymptotic estimate for $\mathcal{L}_{g} \Theta_{k}$ : for any sequence $\left(x_{k}\right)_{k}$ of points in $M$ satisfying $\delta_{k}<<d_{g}\left(x_{k}, \xi_{k}\right) \leq \delta / 4$ there holds:

$$
\begin{aligned}
\mathcal{L}_{\xi} \Theta_{k}\left(x_{k}\right)_{i j}= & K(n) f\left(\xi_{k}\right)^{-\frac{n}{2}}\left[\delta_{i j} \zeta^{p} \check{x}_{p}-\zeta_{i} \check{x}_{j}-\zeta_{j} \check{x}_{i}-(n-2) \zeta^{p} \check{x}_{p} \check{x}_{i} \check{x}_{j}\right] \\
& \times\left(\left|X\left(\xi_{k}\right)\right|_{g}+O\left(\delta_{k}\|\nabla X\|_{L^{\infty}\left(2 r_{k}\right)}\right)\right) d_{g}\left(\xi_{k}, x_{k}\right)^{1-n}(1+o(1)),
\end{aligned}
$$

where we have let, up to a subsequence:

$$
\check{x}=\lim _{k \rightarrow \infty} \frac{1}{d_{g}\left(\xi_{k}, x_{k}\right)} \exp _{\xi_{k}}^{-1}\left(x_{k}\right), \quad K(n)=\frac{n^{\frac{n+2}{2}}(n-2)^{\frac{n}{2}} \omega_{n}}{2^{n+1}(n-1) \omega_{n-1}} \text { and } \zeta=\lim _{k \rightarrow \infty} \frac{X\left(\xi_{k}\right)}{\left|X\left(\xi_{k}\right)\right|_{g}} .
$$

We conclude this section with a refinement of Proposition 9.2 which takes into account the behavior of $v_{k}$ "far away" from the concentration point $\xi_{k}$.

Proposition 9.3. Let $T_{k}, \Theta_{k}$ and $T$ be as in (9.9). Let $\left(R_{k}\right)_{k}$ be a sequence of positive numbers such that $1 \leq R_{k}=o\left(\delta_{k}^{-\frac{1}{2}}\right)$ as $k \rightarrow+\infty$. There holds, for any $x \in M$ :

$$
\begin{aligned}
\left|\mathcal{L}_{g} T_{k}-\mathcal{L}_{g} T\right|_{g}(x) \leq & C\left(\left[\left|X\left(\xi_{k}\right)\right|_{g}+\delta_{k}\|\nabla X\|_{L^{\infty}\left(2 r_{k}\right)}\right] \theta_{k}(x)^{1-n}+\delta_{k}^{\frac{n}{2}} \theta_{k}(x)^{1-n}\right. \\
& \left.+\|X\|_{C^{0}(M)} \varepsilon_{k} \delta_{k} R_{k}^{2}+\|X\|_{C^{0}(M)}\left\|v_{k}\right\|_{L^{\infty}\left(M \backslash B_{\xi_{k}}\left(R_{k} \sqrt{\delta_{k}}\right)\right)}\right),
\end{aligned}
$$

where $C$ is some positive constant that does not depend on $k$, on $\|X\|_{C^{0}(M)}$ and on the choice of $\left(R_{k}\right)_{k}$.

Proof. Let again $G_{i}, 1 \leq i \leq n$, be the Green fields for $\vec{\triangle}_{g}$ satisfying (9.3) in $B_{\xi_{k}}(\delta)$. Mimicking what we did in (9.12), we define:

$$
\begin{aligned}
& Q_{k}^{1}(x)_{i}=\left(\int_{B_{x_{0}}\left(\frac{\delta}{2}\right)}\left\langle G_{i}(x, y),\left[\left(u+W_{k}+v_{k}\right)^{2^{*}}-\left(u+v_{k}\right)^{2^{*}}\right](y) X(y)\right\rangle_{g(y)} d v_{g}(y)\right) \times \eta\left(d_{g}\left(\xi_{k}, x\right)\right), \\
& Q_{k}^{2}(x)_{i}=\left(\int_{B_{x_{0}}\left(\frac{\delta}{2}\right)}\left\langle G_{i}(x, y),\left[\left(u+v_{k}\right)^{2^{*}}-u^{2^{*}}\right](y) X(y)\right\rangle_{g(y)} d v_{g}(y)\right) \times \eta\left(d_{g}\left(\xi_{k}, x\right)\right) .
\end{aligned}
$$

The term $\left(u+W_{k}+v_{k}\right)^{2^{*}}-\left(u+v_{k}\right)^{2^{*}}$ is compactly supported in $B_{\xi_{k}}\left(2 r_{k}\right)$ and satisfies there:

$$
\left|\left(u+W_{k}+v_{k}\right)^{2^{*}}-\left(u+v_{k}\right)^{2^{*}}-W_{k}^{2^{*}}\right| \leq C\left(W_{k}^{2^{*}-1}+W_{k}\right) .
$$

Therefore, (9.4) and Giraud's lemma show that there holds, for any $x \in M$ :

$$
\left|Q_{k}^{1}\right|_{g}+\theta_{k}(x)\left|\mathcal{L}_{g} Q_{k}^{1}\right|_{g} \leq\left[\left|X\left(\xi_{k}\right)\right|_{g}+\delta_{k}\|\nabla X\|_{L^{\infty}\left(2 r_{k}\right)}\right] \theta_{k}(x)^{2-n} .
$$

We thus obtain that there holds:

$$
\vec{\triangle}_{g}\left(T_{k}-Q_{k}^{1}-T\right)=\left\{\begin{array}{lr}
{\left[\left(u+v_{k}\right)^{2^{*}}-u^{2^{*}}\right] X} & \text { in } B_{\xi_{k}}\left(\frac{\delta}{4}\right), \\
{\left[\left(u+v_{k}\right)^{2^{*}}-u^{2^{*}}\right] X+O\left(\left|X\left(\xi_{k}\right)\right|_{g}+\delta_{k}\|\nabla X\|_{L^{\infty}\left(2 r_{k}\right)}\right)} & \text { in } M \backslash B_{\xi_{k}}\left(\frac{\delta}{4}\right) .
\end{array}\right.
$$


We now estimate $Q_{k}^{2}$ in (9.19). With (9.8) we write that there holds, for some positive $C$ :

$$
\left|\left(u+v_{k}\right)^{2^{*}}-u^{2^{*}}\right| \leq C \times\left\{\begin{array}{l}
\varepsilon_{k}^{2^{*}} W_{k}^{2^{*}}+1 \text { in } B_{\xi_{k}}\left(\sqrt{\delta_{k}}\right), \\
\varepsilon_{k} \text { in } B_{\xi_{k}}\left(R_{k} \sqrt{\delta_{k}}\right) \backslash B_{\xi_{k}}\left(\sqrt{\delta_{k}}\right), \\
\left\|v_{k}\right\|_{L^{\infty}\left(M \backslash B_{\xi_{k}}\left(R_{k} \sqrt{\delta_{k}}\right)\right)} \text { in } M \backslash B_{\xi_{k}}\left(R_{k} \sqrt{\delta_{k}}\right) .
\end{array}\right.
$$

Then (9.4) and Giraud's lemma yield again, for any $x \in M$ :

$$
\begin{aligned}
\left|Q_{k}^{2}\right|_{g}(x)+\theta_{k}(x)\left|\mathcal{L}_{g} Q_{k}^{2}\right|_{g}(x) \leq & C\left(\varepsilon_{k}^{2^{*}}\left[\left|X\left(\xi_{k}\right)\right|_{g}+\delta_{k}\|\nabla X\|_{L^{\infty}\left(2 r_{k}\right)}\right] \theta_{k}(x)^{2-n}+\delta_{k}^{\frac{n}{2}} \theta_{k}(x)^{2-n}\right. \\
& \left.+\|X\|_{C^{0}(M)} \varepsilon_{k} R_{k}^{2} \delta_{k}+\|X\|_{C^{0}(M)}\left\|v_{k}\right\|_{L^{\infty}\left(M \backslash B_{\xi_{k}}\left(R_{k} \sqrt{\delta_{k}}\right)\right)}\right)
\end{aligned}
$$

so that there holds, with (9.21):

$$
\vec{\triangle}_{g}\left(T_{k}-Q_{k}^{1}-Q_{k}^{2}-T\right)=\left\{\begin{array}{l}
0 \text { in } B_{\xi_{k}}(\delta / 4) \\
O\left(\left|X\left(\xi_{k}\right)\right|_{g}+\delta_{k}\|\nabla X\|_{L^{\infty}\left(2 r_{k}\right)}+\delta_{k}^{\frac{n}{2}}+\|X\|_{C^{0}(M)} \varepsilon_{k} R_{k}^{2} \delta_{k}\right. \\
\left.+\|X\|_{C^{0}(M)}\left\|v_{k}\right\|_{L^{\infty}\left(M \backslash B_{\xi_{k}}\left(R_{k} \sqrt{\delta_{k}}\right)\right)}\right) \text { in } M \backslash B_{\xi_{k}}(\delta / 4)
\end{array}\right.
$$

The Claim then follows from (9.23), standard elliptic theory, (9.20) and (9.22).

\section{REFERENCES}

1. Antonio Ambrosetti and Andrea Malchiodi, Perturbation methods and semilinear elliptic problems on $\mathbf{R}^{n}$, Progress in Mathematics, vol. 240, Birkhäuser Verlag, Basel, 2006. MR 2186962 (2007k:35005)

2. Robert Bartnik and Jim Isenberg, The constraint equations, The Einstein equations and the large scale behavior of gravitational fields, Birkhäuser, Basel, 2004, pp. 1-38. MR 2098912 (2005j:83007)

3. Robert Beig, Piotr T. Chruściel, and Richard Schoen, KIDs are non-generic, Ann. Henri Poincaré 6 (2005), no. 1, 155-194. MR 2121280 (2005m:83013)

4. Massimiliano Berti and Andrea Malchiodi, Non-compactness and multiplicity results for the yamabe problem on $\mathbb{S}^{n}$, J. Funct. Anal. 180 (2001), 210-241.

5. Gabriele Bianchi and Henrik Egnell, A note on the Sobolev inequality, J. Funct. Anal. 100 (1991), no. 1, $18-24$. MR 1124290 (92i:46033)

6. Piotr Bizoń, Stefan Pletka, and Walter Simon, Initial data for rotating cosmologies, Classical Quantum Gravity 32 (2015), no. 17, 175015, 21. MR 3388125

7. Simon Brendle, Blow-up phenomena for the Yamabe equation, J. Amer. Math. Soc. 21 (2008), no. 4, 951-979. MR 2425176 (2009m:53084)

8. Simon Brendle and Fernando C. Marques, Blow-up phenomena for the Yamabe equation. II, J. Differential Geom. 81 (2009), no. 2, 225-250. MR 2472174 (2010k:53050)

9. Piotr T. Chruściel and Romain Gicquaud, Bifurcating solutions of the lichnerowicz equation, (2015), Preprint.

10. Manuel del Pino, Patricio Felmer, and Monica Musso, Two-bubble solutions in the super-critical Bahri-Coron's problem, Calc. Var. Partial Differential Equations 16 (2003), no. 2, 113-145. MR 1956850

11. Manuel del Pino, Monica Musso, and Frank Pacard, Bubbling along boundary geodesics near the second critical exponent, J. Eur. Math. Soc. (JEMS) 12 (2010), no. 6, 1553-1605. MR 2734352 (2012a:35115)

12. Manuel del Pino, Monica Musso, Frank Pacard, and Angela Pistoia, Large energy entire solutions for the Yamabe equation, J. Differential Equations 251 (2011), no. 9, 2568-2597. MR 2825341 (2012k:35176)

13. Olivier Druet, From one bubble to several bubbles: the low-dimensional case, J. Differential Geom. 63 (2003), no. 3, 399-473. MR 2015469

14. Compactness for Yamabe metrics in low dimensions, Int. Math. Res. Not. (2004), no. 23, $1143-1191$. MR 2041549 (2005b:53056)

15. __ La notion de stabilité pour des équations aux dérivées partielles elliptiques, Ensaios Matemáticos [Mathematical Surveys], vol. 19, Sociedade Brasileira de Matemática, Rio de Janeiro, 2010. MR 2815304 
16. Olivier Druet and Emmanuel Hebey, Stability and instability for Einstein-scalar field Lichnerowicz equations on compact Riemannian manifolds, Math. Z. 263 (2009), no. 1, 33-67. MR 2529487 (2010h:58028)

17. _ Stability for strongly coupled critical elliptic systems in a fully inhomogeneous medium, Anal. PDE 2 (2009), no. 3, 305-359. MR 2603801

18. Olivier Druet, Emmanuel Hebey, and Frédéric Robert, Blow-up theory for elliptic PDEs in Riemannian geometry, Mathematical Notes, vol. 45, Princeton University Press, Princeton, NJ, 2004. MR 2063399 (2005g:53058)

19. Olivier Druet, Emmanuel Hebey, and Jérôme Vétois, Bounded stability for strongly coupled critical elliptic systems below the geometric threshold of the conformal Laplacian, J. Funct. Anal. 258 (2010), no. 3, 999-1059. MR 2558186 (2011a:53053)

20. _ Static klein-gordon-maxwell-proca systems in 4-dimensional closed manifolds. ii, J. Reine Angew. Math. (2015), To appear.

21. Olivier Druet and Bruno Premoselli, Stability of the Einstein-Lichnerowicz constraint system, Math. Ann. 362 (2015), no. 3-4, 839-886. MR 3368085

22. Louis Dupaigne, Stable solutions of elliptic partial differential equations, Chapman \& Hall/CRC Monographs and Surveys in Pure and Applied Mathematics, vol. 143, Chapman \& Hall/CRC, Boca Raton, FL, 2011. MR 2779463

23. Pierpaolo Esposito, Angela Pistoia, and Jérôme Vétois, The effect of linear perturbations on the Yamabe problem, Math. Ann. 358 (2014), no. 1-2, 511-560. MR 3158007

24. Romain Gicquaud and Cang Nguyen, Solutions to the Einstein-scalar field constraint equations with a small TT-tensor, Calc. Var. Partial Differential Equations 55 (2016), no. 2, 55:29. MR 3466902

25. Emmanuel Hebey, Compactness and stability for nonlinear elliptic equations, Zurich Lectures in Advanced Mathematics, European Mathematical Society (EMS), Zürich, 2014. MR 3235821

26. Emmanuel Hebey, Frank Pacard, and Daniel Pollack, A variational analysis of Einstein-scalar field Lichnerowicz equations on compact Riemannian manifolds, Comm. Math. Phys. 278 (2008), no. 1, 117-132. MR 2367200 (2009c:58041)

27. Emmanuel Hebey and Frédéric Robert, Asymptotic analysis for fourth order Paneitz equations with critical growth, Adv. Calc. Var. 4 (2011), no. 3, 229-275. MR 2819586 (2012h:58045)

28. Emmanuel Hebey and Giona Veronelli, The Lichnerowicz equation in the closed case of the Einstein-Maxwell theory, Trans. Amer. Math. Soc. 366 (2014), no. 3, 1179-1193. MR 3145727

29. Emmanuel Hebey and Juncheng Wei, Schrödinger-Poisson systems in the 3-sphere, Calc. Var. Partial Differential Equations 47 (2013), no. 1-2, 25-54. MR 3044130

30. M. Holst and C. Meier, Non uniqueness of solutions to the conformal formulation, arXiv:1210.2156.

31. M. A. Khuri, F. C. Marques, and R. M. Schoen, A compactness theorem for the Yamabe problem, J. Differential Geom. 81 (2009), no. 1, 143-196. MR 2477893 (2010e:53065)

32. John M. Lee and Thomas H. Parker, The Yamabe problem, Bull. Amer. Math. Soc. (N.S.) 17 (1987), no. 1, 37-91. MR 888880 (88f:53001)

33. Yan Yan Li and Lei Zhang, Compactness of solutions to the Yamabe problem. III, J. Funct. Anal. 245 (2007), no. 2, 438-474. MR 2309836 (2008f:53038)

34. Yanyan Li and Meijun Zhu, Yamabe type equations on three-dimensional Riemannian manifolds, Commun. Contemp. Math. 1 (1999), no. 1, 1-50. MR 1681811 (2000m:53051)

35. Li Ma and Juncheng Wei, Stability and multiple solutions to Einstein-scalar field Lichnerowicz equation on manifolds, J. Math. Pures Appl. (9) 99 (2013), no. 2, 174-186. MR 3007843

36. Fernando Coda Marques, A priori estimates for the Yamabe problem in the non-locally conformally flat case, J. Differential Geom. 71 (2005), no. 2, 315-346. MR 2197144 (2006i:53046)

37. Bruno Premoselli, The Einstein-Scalar Field Constraint System in the Positive Case, Comm. Math. Phys. 326 (2014), no. 2, 543-557. MR 3165467

38. — Effective multiplicity for the Einstein-scalar field Lichnerowicz equation, Calc. Var. Partial Differential Equations 53 (2015), no. 1-2, 29-64. MR 3336312

39. - Stability and instability for the einstein-lichnerowicz constraints system, Int. Math. Res. Not. IMRN (2015), Published online.

40. Bruno Premoselli and Juncheng Wei, Non-compactness and infinite number of conformal initial data sets in high dimensions, J. Funct. Anal. 270 (2016), no. 2, 718-747. MR 3425901

41. Olivier Rey, The role of the Green's function in a nonlinear elliptic equation involving the critical Sobolev exponent, J. Funct. Anal. 89 (1990), no. 1, 1-52. MR 1040954 (91b:35012)

42. Olivier Rey and Juncheng Wei, Arbitrary number of positive solutions for an elliptic problem with critical nonlinearity, J. Eur. Math. Soc. (JEMS) 7 (2005), no. 4, 449-476. MR 2159223

43. Frédéric Robert, Existence et asymptotiques optimales des fonctions de green des opérateurs elliptiques d'ordre deux, http://www.iecn.u-nancy.fr/ frobert/ConstrucGreen.pdf. 
44. Frédéric Robert and Jérôme Vétois, Examples of non-isolated blow-up for perturbations of the scalar curvature equation on non-locally conformally flat manifolds, J. Differential Geom. 98 (2014), no. 2, 349-356. MR 3263521

45. _ A general theorem for the construction of blowing-up solutions to some elliptic nonlinear equations with lyapunov-schmidt's finite-dimensional reduction, Concentration Compactness and Profile Decomposition (Bangalore, 2011), Trends in Mathematics, Springer, Basel (2014), 85-116.

46. Sign-changing solutions to elliptic second order equations: glueing a peak to a degenerate critical manifold, Calc. Var. Partial Differential Equations 54 (2015), no. 1, 693-716. MR 3385177

47. Michael Struwe, A global compactness result for elliptic boundary value problems involving limiting nonlinearities, Math. Z. 187 (1984), no. 4, 511-517. MR 760051

48. Juncheng Wei, On the construction of single-peaked solutions to a singularly perturbed semilinear Dirichlet problem, J. Differential Equations 129 (1996), no. 2, 315-333. MR 1404386

49. - On the boundary spike layer solutions to a singularly perturbed Neumann problem, J. Differential Equations 134 (1997), no. 1, 104-133. MR 1429093

50. - On single interior spike solutions of the Gierer-Meinhardt system: uniqueness and spectrum estimates, European J. Appl. Math. 10 (1999), no. 4, 353-378. MR 1713076

51. — Existence and stability of spikes for the Gierer-Meinhardt system, Handbook of differential equations: stationary partial differential equations. Vol. V, Handb. Differ. Equ., Elsevier/North-Holland, Amsterdam, 2008, pp. 487-585. MR 2497911 (2011b:35214)

Bruno Premoselli, Université Libre de Bruxelles, Service de Géométrie Différentielle, CP 218, Boulevard du Triomphe, B-1050 Bruxelles, Belgique.

E-mail address: bruno.premoselli@ulb.ac.be 